\title{
Chemogenetic modulation of sensory neurons reveals their regulating role in melanoma progression
}

\author{
Pedro A. C. Costa', Walison N. Silva', Pedro H. D. M. Prazeres' ${ }^{1}$, Caroline C. Picoli' , Gabriela D. A. Guardia², \\ Alinne C. Costa', Mariana A. Oliveira ${ }^{3}$, Pedro P. G. Guimarães ${ }^{4}$, Ricardo Gonçalves ${ }^{1}$, Mauro C. X. Pinto ${ }^{5}$, \\ Jaime H. Amorim ${ }^{6}$, Vasco A. C. Azevedo ${ }^{7}$, Rodrigo R. Resende ${ }^{3}$, Remo C. Russo ${ }^{4} \mathbb{C}^{\text {, Thiago M. Cunha }}{ }^{8}$, \\ Pedro A. F. Galante ${ }^{2}$, Akiva Mintz ${ }^{9}$ and Alexander Birbrair ${ }^{1,9^{*}}$ (D)
}

\begin{abstract}
Sensory neurons have recently emerged as components of the tumor microenvironment. Nevertheless, whether sensory neuronal activity is important for tumor progression remains unknown. Here we used Designer Receptors Exclusively Activated by a Designer Drug (DREADD) technology to inhibit or activate sensory neurons' firing within the melanoma tumor. Melanoma growth and angiogenesis were accelerated following inhibition of sensory neurons' activity and were reduced following overstimulation of these neurons. Sensory neuron-specific overactivation also induced a boost in the immune surveillance by increasing tumor-infiltrating anti-tumor lymphocytes, while reducing immune-suppressor cells. In humans, a retrospective in silico analysis of melanoma biopsies revealed that increased expression of sensory neurons-related genes within melanoma was associated with improved survival. These findings suggest that sensory innervations regulate melanoma progression, indicating that manipulation of sensory neurons' activity may provide a valuable tool to improve melanoma patients' outcomes.
\end{abstract}

Keywords: Sensory neurons, Tumor microenvironment, Melanoma, Neuronal activity, Chemogenetics

\section{Introduction}

Melanoma represents one of the leading causes of cancer-related deaths, being the most aggressive skin cancer type worldwide [124]. It emerges from molecularly altered melanocytes, which are the producers of melanin in the skin [48]. These cancer cells are embedded within the cutaneous microenvironment where they reside and interact dynamically with its constituents during disease progression $[15,54]$. Understanding the interplay between the different components within the tumor microenvironment is crucial for the success

*Correspondence: birbrair@icb.ufmg.br

${ }^{1}$ Departamento de Patologia, Universidade Federal de Minas Gerais, Belo Horizonte, MG, Brasil

Full list of author information is available at the end of the article of therapeutic applications, since each component can be influenced by the others, resulting in impacts on the cancer cells $[9,10,45,52,88,106]$. The presence of individual nerve fibers within the tumor microenvironment was ignored for many years as they are difficult to detect in classical histology. For a long time, only large nerve trunks were detected within tumors, and they were always associated with perineural invasion of cancer cells, a process in which these cells grow and migrate along native passive tissue nerves [84]. Recently, a different phenomenon was described, by which the tumor itself is infiltrated pro-actively by newly developed peripheral nerve projections [32, 36, 71, 89, 108, 115, 116, 121, 154, 158].

To understand how peripheral innervations behave within the tumors, functional studies, in which original author(s) and the source, provide a link to the Creative Commons licence, and indicate if changes were made. The images or other third party material in this article are included in the article's Creative Commons licence, unless indicated otherwise in a credit line to the material. If material is not included in the article's Creative Commons licence and your intended use is not permitted by statutory regulation or exceeds the permitted use, you will need to obtain permission directly from the copyright holder. To view a copy of this licence, visit http://creativecommons.org/licenses/by/4.0/. The Creative Commons Public Domain Dedication waiver (http://creativeco mmons.org/publicdomain/zero/1.0/) applies to the data made available in this article, unless otherwise stated in a credit line to the data. 
intra-tumoral nerves were eliminated, have relied on the surgical or pharmacological manipulation of nerves. Each such strategy, however, has its disadvantages. Peripheral nerves contain mixtures of different nerve fiber types [41, 82], and therefore, surgical denervation of a peripheral nerve leads to the disruption of all the nerve fibers present within that specific nerve [101]. Consequently, the role of particular nerve projections in the tumor cannot be isolated, as other nerve fibers are also affected. On the other hand, pharmacological drugs cause systemic reactions in several organs and indirect effects on unexpected targets. Thus, achieving the neuronal type-specificity that is needed to understand the role that specific nerve fibers perform in the tumor microenvironment is difficult with these methods, and the observed outcomes could be due to the unwanted effects on other innervations in addition to the targeted neurons. Wherefore, conclusions drawn from studies based on surgical or pharmacological denervation may be imprecise. These are some of the reasons, in addition to tumor tissue specificity, for some of the ambiguity about the roles of specific nerve fibers in cancer behavior. Accordingly, contradictory reports have been published: while some studies have claimed that certain neuronal types promote cancer progression [57, $158]$, others concluded that they suppress tumorigenesis $[32,116]$.

Therefore, to study the role of specific innervations, these should be directly manipulated in a nerve-fibertype-specific manner. Recently, this approach became possible with the advent of powerful genetically-based tools, that precisely allow the targeting and elimination of specific peripheral nerve fibers for studying their functions in vivo [13]. Our group showed that specific genetic depletion of sensory neurons promotes melanoma growth [108]. Nevertheless, genetic ablation of these innervations may result in the generation of a proinflammatory microenvironment, secondary to cell death in the site where the neurons were ablated (Männ et al. 2016; Christiaansen, Boggiatto, and Varga 2014; Bennett et al. 2005), which itself is strongly tumor growth promoting $[49,67]$, and can affect cancer cells' behavior [50]. Thus, it remains unclear which facets of the sensory neuron-ablated tumor phenotype are due to the loss of sensory innervations, rather than indirect effects due to the local inflammation caused by the death of these neurons. To circumvent this issue, in the present study, we used chemogenetics, an experimental strategy that has empowered neuroscience studies [131, 147], to determine the precise role of sensory neurons in the regulation of melanoma progression. Designer Receptors Exclusively Activated by Designer Drugs (DREADDs) enable the silencing or overactivation of genetically defined neuronal populations upon binding to small-molecule designer drugs [119]. This approach allowed for highly selective and non-invasive modulation of sensory neurons' activity in the tumor. Here, we revealed that silencing of sensory neurons' activity, without ablating them, is sufficient to trigger increase in melanoma growth and in intra-tumoral new blood vessel formation. In contrast, chemogenetic stimulation of sensory neurons counteracted melanoma progression, by regulating tumoral growth, angiogenesis and immunosurveillance. Our results provide unequivocal evidence of the influence of sensory neurons in cancer progression.

\section{Materials and methods}

\section{Animals}

Generation of Nav1.8-Cre mice, in which Nav1.8+ sensory neurons express Cre recombinase, have been previously described. These animals were obtained from Infrafrontier (EMMA ID: 04 582). R26-LSL-hM4DiDREADD (hM4Di) and CAG-LSL-hM3Dq-DREADD (hM3Dq) mice were purchased from the Jackson Laboratory (Jax) (Bar Harbor, ME).

To silence neuronal activity in sensory innervations in vivo, Nav1.8-Cre mice were crossed with R26-LSLhM4Di-DREADD (hM4Di), a mouse line conditionally expressing a Gi-coupled engineered human muscarinic 4 receptor (hM4Di) [159]. hM4Di is a mutant G protein-coupled receptor which induces the canonical Gi pathway following binding to the pharmacologically inert drug clozapine- $N$-oxide (CNO). In Nav1.8-Cre + I hM4Di + mice, upon removal of the loxP-stop-loxP cassette by Cre recombination, the Gi-coupled hM4Di is expressed only in Nav1.8+ sensory neurons. Thus, sensory neuronal activity can be silenced by the administration of CNO. Nav1.8-Cre-/hM4Di + mice were used as controls.

To promote sensory neuron overactivation in vivo, Nav1.8-Cre mice were crossed with CAG-LSL-hM3DqDREADD (hM3Dq) animals, a mouse line conditionally expressing an evolved $\mathrm{Gq}$ protein-coupled receptor (hM3Dq), to generate Nav1.8-Cre $+/ \mathrm{hM} 3 \mathrm{Dq}+$ mice. In Nav1.8-Cre +/hM3Dq + animals, upon removal of loxPstop-loxP cassette by Cre recombination, the Gq-coupled hM3Dq is expressed specifically in Nav1.8-sensory neurons. $\mathrm{hM} 3 \mathrm{Dq}$ is a mutant $\mathrm{G}$ protein-coupled receptor which induces the canonical Gq pathway following the binding to $\mathrm{CNO}$. Thus, sensory neuron firing can be chemically induced by administration of CNO. Nav1.8Cre-/ hM3Dq + animals were used as controls.

All animal care and experimental procedures were approved by the Ethics Animal Care and Use Committee (CEUA), in accordance with the Guide for the Care and Use of Laboratory Animals from the Federal University of Minas Gerais. All colonies were housed in 
a pathogen-free animal facility of the Department of Pathology, UFMG, under controlled light cycle (12:12-h light/dark cycle) and fed ad libitum. Age-matched 8- to 12 -week-old mice were used for all experiments. All experiments used mice heterozygous for both NaV1.8Cre and DREADD receptors.

\section{Cell culture}

Murine B16-F10 melanoma is a common cell line that naturally originated in melanin-producing epithelia of C57BL6 mice. These cells were originally obtained from ATCC (USA), and were used to study melanoma development in vivo. The cells were cultured in Dulbecco's modified Eagle's medium (DMEM) supplemented with $10 \%(\mathrm{v} / \mathrm{v})$ fetal calf serum $/ 2 \mathrm{mM} \mathrm{L}$-glutamine $/ 100 \mathrm{U} / \mathrm{ml}$ penicillin $/ 100 \mu \mathrm{g} / \mathrm{ml}$ streptomycin. The cells have been tested and found negative for mycoplasma. Cells were cultured in a humidified atmosphere of $95 \%$ air and 5\% (v/v) $\mathrm{CO} 2$ at $37^{\circ} \mathrm{C}$.

\section{Melanoma tumor implantation}

B16-F10 cells were suspended in PBS and examined for viability using trypan blue staining. B16-F10 cells were used for transplantation only when more than $90 \%$ of cells were viable. For subcutaneous injection, the skin of all mice at an age of 8-12 weeks was shaved at the place of application. $1 \times 10^{5}$ cells in $100 \mu \mathrm{L}$ were injected subcutaneously into the right flank of each mouse and the growth of the tumors was monitored until sacrifice. Growth of the tumors was assessed over time with a caliper as previously reported [12]. For determination of tumor volume, tumor-bearing animals were anesthetized with isoflurane in $\mathrm{O} 2$ by manually restraining the mice and placing their heads in in-house-built nose cones. Tumors were removed 16 days after transplantation and weighted. Length (L) and width (W) were calculated to measure tumor volume $(\mathrm{V})$ using the formula $\mathrm{V}=0.5 \times(\mathrm{L} \times \mathrm{W} 2)$ [40]. Tumor area was determined using calibrated photographs of each tumor using Fiji software $^{\circledR}$, version 1.53 (National Institute of Health, Bethesda, MD).

\section{CNO treatment}

The DREADD ligand clozapine-N-oxide (CNO) $(1 \mathrm{mg} /$ $\mathrm{kg}$ in saline) (Sigma-Aldrich, St Louis, MO, USA) [7] was administered intra-peritoneally using a 25 -gauge needle daily to test the effect of neuronal inhibition or activation on melanoma progression in Nav1.8-Cre +/hM4Di + and Nav1.8-Cre +/hM3Dq + animals, respectively. Control Nav1.8-Cre-/hM4Di + and Nav1.8-Cre-/hM3Dq + mice were similarly injected with $\mathrm{CNO}$.

\section{Capsaicin-induced spontaneous behavior}

To confirm sensory neurons inhibition efficiency, following acclimation, Nav1.8-Cre-/hM4Di + and Nav1.8-Cre +/hM4Di + mice were injected with an intra-plantar subcutaneous dose of $10 \mu \mathrm{l}$ of capsaicin $(1 \mu \mathrm{g} / 10 \mu \mathrm{l}$; Sigma-Aldrich). A video recording was taken for $5 \mathrm{~min}$ post-capsaicin injection. The time that the animals spent performing spontaneous behaviors of licking, lifting, and flicking the paw were measured for $5 \mathrm{~min}$ after injection of capsaicin from these videos.

\section{Immunohistochemistry and microscopy}

Adult mice were deeply anesthetized with isoflurane and transcardially perfused with saline followed by $4 \%$ buffered paraformaldehyde (PFA, $\mathrm{pH}=7.4$ ). After dissection, B16F10 tumors were fixed overnight at $4{ }^{\circ} \mathrm{C}$ in $4 \%$ buffered paraformaldehyde, incubated overnight at $4{ }^{\circ} \mathrm{C}$ with $30 \%$ sucrose diluted in PBS, embedded and frozen in optimal cutting temperature compound (OCT, TissueTek). Embedded tumors were stored at $-80{ }^{\circ} \mathrm{C} .20 \mu \mathrm{m}$ cryosections were cut and blocked for $2 \mathrm{~h}$ in $3 \% \mathrm{BSA}$ in PBS $+0.5 \%$ Triton and immunostained with the following antibodies: CD31-PE (dilution 1:100) (BioLegend), Ki67 (dilution 1:100) (BD Biosciences), and anti-Guinea pig-AlexaFluor-647 (1:1000) (Life Technologies) [10, 23]. After this, the sections were washed with PBS containing 4,6-diamidino-2-phenylindole (DAPI, $5 \mu \mathrm{g} / \mathrm{ml}$, Invitrogen) and mounted using Dako fluorescence mounting medium (Dako, Santa Clara, CA). Stained tumor sections were imaged and analyzed by confocal microscopy using an inverted Zeiss LSM 880 confocal microscope (Oberkochen, Germany). CD31 area and the number of $\mathrm{Ki} 67^{+}$cells were quantified using Fiji software ${ }^{\circledR}$, version 1.53 (National Institute of Health). Multiple random fields of each section were used for quantification.

\section{Tumor-infiltrating leukocytes immunophenotyping and intracellular cytokine measurement}

Tumors, their draining lymph nodes and non-tumor draining lymph nodes were harvested. Tissues were macerated and filtrated trough cell strainers of 40 um (Falcon) to isolate the cells used for immunophenotyping. Cells were washed in phosphate-buffered saline (PBS), incubated with Live/Dead solution (Invitrogen), for dead cell exclusion, and with monoclonal antibodies, washed, fixed, and permeabilized (FoxP3 staining buffer set, eBioscience) according to manufacture's instructions. Antibodies are listed in Table 1. Acquisition was realized on a LSR-FORTESSA. For analyses, to exclude debris, combinations of fluorochromes was done, to remove doublets a forward scatter area (FSC-A) versus forward scatter height (FSC-H) gate was used, and then cells were gated in function of time versus FSC-A to 
Table 1 Antibodies used in flow cytometry

\begin{tabular}{|c|c|c|c|}
\hline Antigen & Fluorochrome & Clone & Company \\
\hline CD3 & eFluor450 & $145-2 C 11$ & ThermoFhisher \\
\hline CD8a & eFluor 450 & $53-6.7$ & ThermoFhisher \\
\hline CD11c & eFluor 450 & N418 & ThermoFhisher \\
\hline LIVE/DEAD & Acqua & & ThermoFhisher \\
\hline Streptavidin & Pacific Orange & & ThermoFhisher \\
\hline CD45 & Super Bright 600 & 30-F11 & ThermoFhisher \\
\hline TCR gamma/delta & Super Bright 645 & eBioGL3 & ThermoFhisher \\
\hline CD4 & Alexa Fluor 488 & GK1.5 & ThermoFhisher \\
\hline $\mathrm{F} 4 / 80$ & FITC & BM8 & Hycult \\
\hline NK1.1 & PE-eFluor 610 & PK136 & ThermoFhisher \\
\hline CD8a & PerCP-Cyanine5.5 & $53-6.7$ & ThermoFhisher \\
\hline Ly-6G & PerCP-eFluor 710 & 1A8-Ly6g & ThermoFhisher \\
\hline IL-17A & PerCP-Cyanine 5.5 & eBio17B7 & ThermoFhisher \\
\hline CTLA-4 & PE-Cyanine7 & UC10-4B9 & ThermoFhisher \\
\hline FoxP3 & Alexa Fluor 647 & $3 G 3$ & ThermoFhisher \\
\hline CD3e & Cyanine5 & $500 A 2$ & ThermoFhisher \\
\hline Ki67 & AlexaFluor 700 & SolA15 & ThermoFhisher \\
\hline PD-1 & APC-eFluor 780 & $J 43$ & ThermoFhisher \\
\hline Ly-6C & APC-eFluor 780 & HK1.4 & ThermoFhisher \\
\hline IFN- $\gamma$ & APC-eFluor 780 & XMG1.2 & ThermoFhisher \\
\hline CD11b & Biotin & $\mathrm{M} 1 / 70$ & Biolegend \\
\hline
\end{tabular}

avoid a possible interference of flux interruptions. Only live leukocytes were used using a Live/Dead gate versus CD45. We assessed different immune cell subpopulations based on molecular markers of each cell subset: $\mathrm{CD} 4+\mathrm{T}$ cells $\left(\mathrm{CD}^{+} / \mathrm{CD}^{+}\right), \mathrm{CD} 8+\mathrm{T}$ cells $\left(\mathrm{CD}^{+} / \mathrm{CD}^{+}\right), \gamma \delta \mathrm{T}$ cells $\left(\mathrm{CD}^{+} / \mathrm{CD}^{-} / \mathrm{CD} 8^{-} / \mathrm{TCR} \gamma \delta^{+}\right)$, NKT cells $\left(\mathrm{CD}^{+} /\right.$ $\mathrm{NK}^{+}$), regulatory $\mathrm{T}$ cells $\left(\right.$ Foxp $\left.3^{+} / \mathrm{CD}^{+} / \mathrm{CD}^{+}\right)$, NK cells

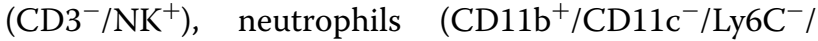

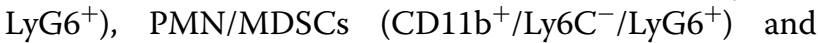
dendritic cells $\left(\mathrm{CD} 11 \mathrm{~b}^{-} / \mathrm{CD} 11 \mathrm{c}^{+} / \mathrm{Ly} 6 \mathrm{C}^{-} / \mathrm{LyG6}^{-}\right)$. In each T-cell subset, frequencies of cells expressing checkpoint inhibitors CTLA-4 and PD1 were evaluated. Cytokine analyses in lymphocytes from the tumor microenvironment and lymph nodes were done using intracellular staining. Briefly, cells were isolated from tumor samples and lymph nodes and cultivated for $4 \mathrm{~h}$ at $37{ }^{\circ} \mathrm{C}$ in $10 \%$ FBS RPMI supplemented with $2 \mathrm{mM}$ L-glutamine, 50 units $/ \mathrm{mL}$ penicillin, and $50 \mu \mathrm{g} / \mathrm{mL}$ streptomycin, in the presence of Brefeldin A (ThermoFisher) and Monensin (ThermoFisher). Following this, cells were washed in FACS buffer and stained for cell surface markers. Cells were then fixed for $35 \mathrm{~min}$ at $4{ }^{\circ} \mathrm{C}$ with eBiosciences Cytofix/Cytoperm buffer and, subsequently, washed once in eBioscience Perm/Wash buffer. Then, cells were stained for $45 \mathrm{~min}$ at $4{ }^{\circ} \mathrm{C}$ with anti-IFN- $\gamma$ and antiIL-17 (Table 1) antibodies diluted in eBioscience Perm/ Wash [78]. Cells were washed twice and the data was acquired. Ki67 is a nuclear factor transcript in the late G1, S, G2, and M of cell cycle, therefore marks proliferating cells $[44,128]$. Thus, we evaluated proliferation in viable CD45 negative cells, suggesting tumoral proliferation. GraphPad Prism V7.0 (GraphPad software) and FlowJo V10.4.11 (TreeStar) were used for data analysis and graphic presentation.

\section{Quantification of CGRP within tumors}

Tumor samples from Nav1.8-Cre $+/ \mathrm{hM} 4 \mathrm{Di}+$ and Nav1.8-Cre +/hM3Dq + animals, as well as from their respective controls (Nav1.8-Cre-/hM4Di+and Nav1.8Cre-/hM3Dq +) were analyzed to measure the amount of CGRP using commercially available Sandwich-CGRP ELISA kit purchased from Elabscience (Catalog \# E-ELM2744). Briefly, tumor pieces were weighed and then homogenized in PBS $(0.01 \mathrm{M}, \mathrm{pH}=7.4)$ with a glass homogenizer on ice. The homogenates were centrifuged for $5 \mathrm{~min}$ at $5000 \times \mathrm{g}$ at $4{ }^{\circ} \mathrm{C}$ to get the supernatant. ELISA of CGRP were performed according to manufacturer's instructions. After ELISA, Optical Density (OD) was measured using Varioskan Flash (Thermo) set at $450 \mathrm{~nm}$.

\section{In silico analysis}

RNA sequencing count data of 103 Skin Cutaneous Melanoma (SKCM) patients was downloaded from The Cancer Genome Atlas (TCGA) repository (https://portal.gdc.cancer.gov/). Differential gene expression analyses were performed between samples of alive and dead patients (considering a 5-year interval) using DESeq2 [83]. We stratified patients in these two groups, alive or dead, based on their vital status in a 5-year interval of their tumor diagnosis (clinical data available at TCGA and curated by Liu et al. (2018) [85]. Genes with absolute $\log 2$ (Fold-change) $\geq 1$ and False Discovery Rate (FDR) adjusted $P$-value $<0.05$ were considered differentially expressed. To identify biological processes associated with genes differentially expressed, we performed a Gene Ontology (GO) enrichment analysis using ShinyGO [42]. We used the STRING database [132] (parameters: full STRING network, considering only text-mining, databases and experiments interactions with score $>0.400$, and only genes with 3 or more interactions) and Cytoscape (https://cytoscape.org/) to construct protein-protein interactions (PPIs) among our manually curated list of 34 gene related to sensory neurons selected based on the literature $[29,37,51,114,141,144]$. The set of 18 genes showing at least two PPI interactions are shown. For the remaining analyses, RNA sequencing counts were first Transcripts Per Million (TPM)-normalized using a local $\mathrm{R}$ script. To identify a gene signature associated with SKCM cancer patient survival we used Reboot [31] with parameters "-B 100 -G 5 -P 0.3 -V 0.01". 
Briefly, Reboot finds genes associated with cancer patient prognosis using multivariate penalized Cox regression combined with a bootstrap approach. In the first step of Reboot, it produces regression coefficients (numerical values) that determine the contribution of each submitted gene to patients' survival. These coefficients may be positive or negative values indicating that high expression of a particular gene potentially contributes to worse or better prognosis, respectively. Once these coefficients are produced, Reboot then calculates the score of each patient (sample) as the sum of each gene coefficient multiplied by the corresponding gene expression level in that patient. Finally, when all patients' scores are calculated, we then stratify them into groups with high/low scores based on the median score of all patients to create the survival curve (Kaplan-Meier). For further information, see [31]. SCN10A box plots and survival curves were created using $\mathrm{R}$ (https://www.r-project.org/) scripts.

\section{Statistical analysis}

Graphs were plotted using GraphPad Prism 7 (San Diego, CA). Shapiro-Wilk normality test was performed, and unpaired $t$ test was used to determine statistical significance.

\section{Results}

\section{Chemogenetic inhibition of Nav1.8 + neurons accelerates} melanoma progression

We have previously demonstrated that melanomas are infiltrated by Nav1.8+ sensory innervations, and that those tumors grow slower when these neurons are pharmacologically or genetically ablated [108]. However, these investigations were performed using Nav1.8Cre $+/ \mathrm{DTA}+$ mice, in which a diphtheria toxin fragment A is constitutively activated in Nav1.8+ sensory neurons, resulting in the toxin induced-death of these cells. Therefore, this technique lacks temporal control of neuronal ablation, and enables compensatory effects during the development of these animals. Importantly, the approach by which specific neurons are ablated from the tissue microenvironment is also limited because of the secondary consequences, such as inflammation, caused in the tissue where sensory neurons are eliminated. Thus, it remains unclear whether these damageinduced changes in the tissue may influence the observed cancer outcomes. Here, we applied a chemogenetic approach to specifically inhibit the activity of Nav1.8expressing sensory neurons without killing these cells. We used DREADDs to specifically control sensory neuron activity. DREADDs are derived from different types of mutated muscarinic receptors that have been engineered to lose affinity to their endogenous ligand acetylcholine [5], but to gain responsiveness to a synthetic ligand, clozapine-N-oxide (CNO). Inhibitory DREADDs (hM4Di) elicit an intracellular cascade that results in the silencing of neuronal activity [113], without changing the number of innervations as previously reported [68]. This method allows for the selective silencing of specific types of neurons in vivo without physical manipulation or destruction in the tissue. DREADDs were expressed specifically in sensory neurons, using a transgenic murine approach: mice carrying the construct encoding for Credependent expression of hM4Di were crossed to Nav1.8Cre animals. In the resulting mice, Nav1.8-Cre+/ hM4Di +, only Nav1.8+ sensory neurons expressed inhibitory DREADDs. As controls in this study, we used littermate mice carrying Cre-dependent hM4Di, but lacking the Cre gene (Nav1.8-Cre-/hM4Di+) (Fig. 1A). This allowed us to control for any potential side effects from CNO administration.

In order to ascertain that the expression of DREADD receptors was driven to intra-tumoral Nav1.8-expressing neurons in Nav1.8-Cre $+/ \mathrm{hM} 3 \mathrm{Di}+$ mice, we used the solid tumor model B16F10. We assessed tumor sections from melanoma grown in Nav1.8-Cre+/TdTomato + mice and detected Nav1.8+ neurons expressing TdTomato present within the tumor microenvironment (Fig. 1B). To validate sensory neuronal inhibition following daily $\mathrm{CNO}$ injection, we used a behavioral test to evaluate the sensitivity to capsaicin, confirming the silencing of sensory neurons, as previously described [2]. Indeed, Nav1.8-Cre $+/ \mathrm{hM} 4 \mathrm{Di}+$ animals spent less time $(25.53 \pm 2.27 \mathrm{~s})$ licking their paws after intra-plantar injection of capsaicin, compared to control animals $(56.53 \pm 3.92 \mathrm{~s})$ (Fig. 1C). To analyze the effect of sensory neurons silencing on tumor growth, we subcutaneously transplanted B16F10 cells to the lower right flank of both inhibitory DREADD-expressing mice (Nav1.8-Cre+/ $\mathrm{hM} 4 \mathrm{Di}+$ ) and their controls (Nav1.8-Cre-/hM4Di+). Following cancer cell injection, we treated the animals daily with $\mathrm{CNO}$ to induce sensory neuronal activity inhibition (controls were also treated with CNO) (Fig. 1D). After 14 days of continuous sensory inhibition, tumor volume was significantly enhanced in the sensory neuron-silenced mice when compared to the controls (tumor volume was increased from $82.1 \pm 29.6$ to $319.6 \pm 72.8$ $\mathrm{mm}^{3}$; Fig. 1E). After 16 days of repeated sensory inhibition, tumor weight was also significantly enhanced in the sensory neuron-silenced mice when compared to the controls (tumor weight was increased from $0.50 \pm 0.04$ to $0.98 \pm 0.23 \mathrm{~g}$; Fig. $1 \mathrm{~F}, \mathrm{G}$ ). Animal weights were not affected by sensory inhibition (data not shown).

Increase in neoangiogenesis within melanoma tumors is correlated with worse outcomes in these patients [111]. We detected, in melanoma-bearing animals with silenced sensory neurons, an enhancement in the 


\section{A}

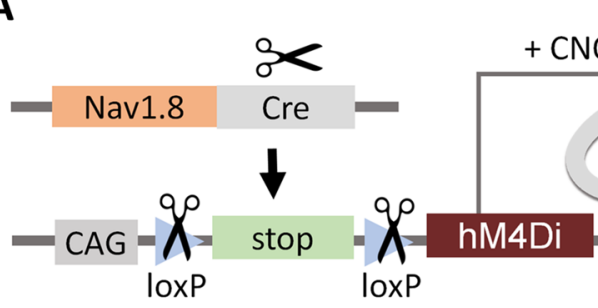

B
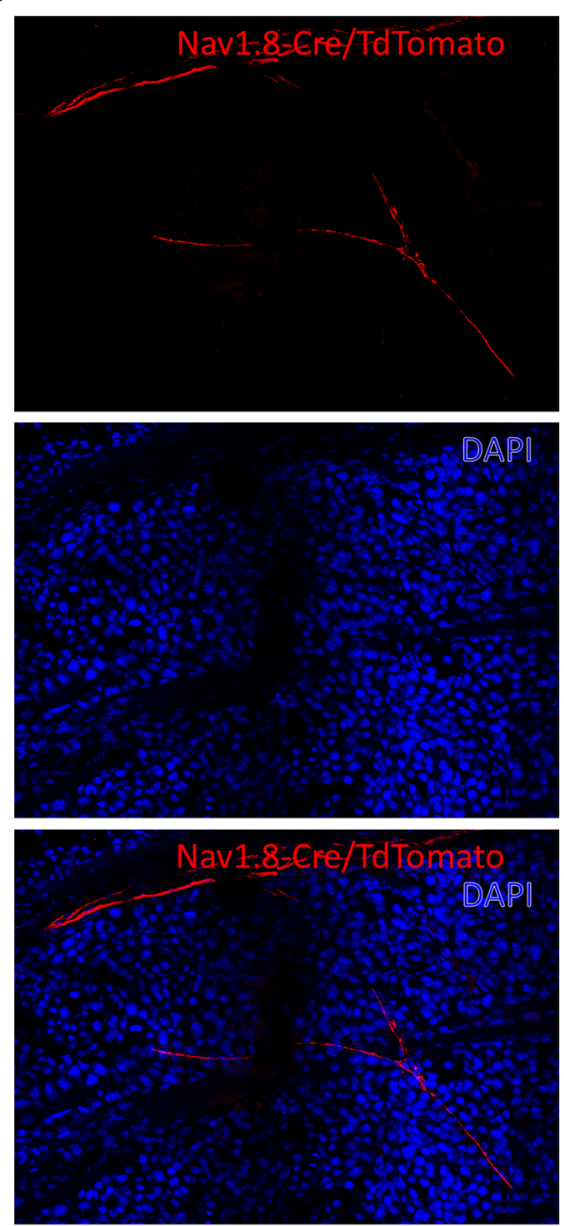

C

$\square$ Nav1.8-Cre-/hM4Di+

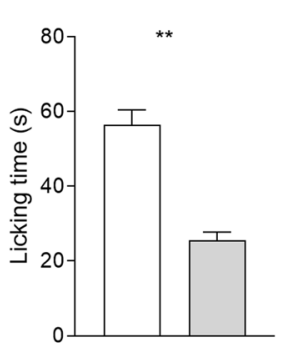

INHIBITION

\section{D $\quad 1 \times 10^{5} \mathrm{~B} 16 \mathrm{~F} 10$ cells s.c.}
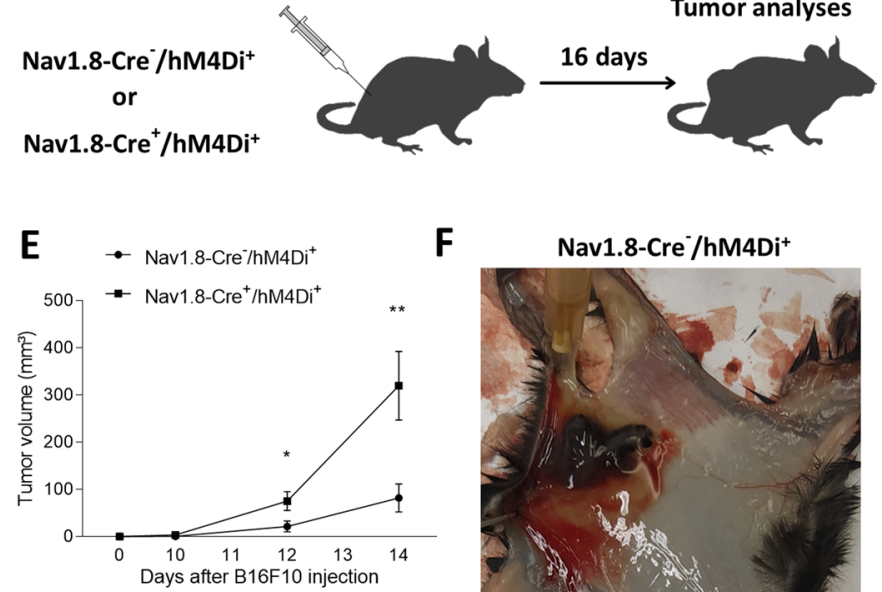

F Nav1.8-Cre $/ \mathrm{hM}^{-} \mathrm{Di}^{+}$

G
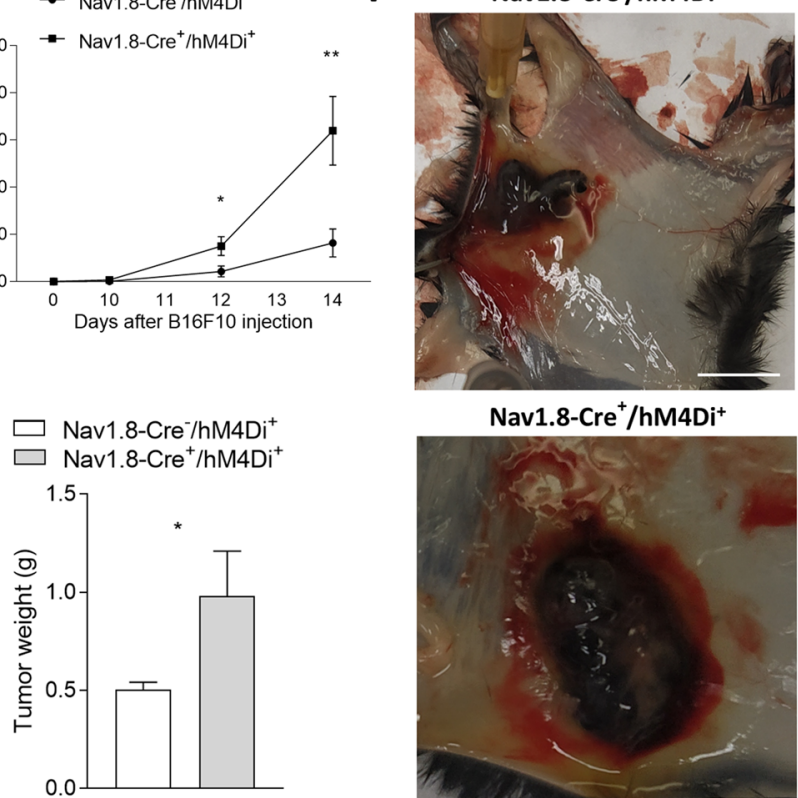

Nav1.8-Cre ${ }^{+} / \mathrm{hM}^{-} \mathrm{Di}^{+}$

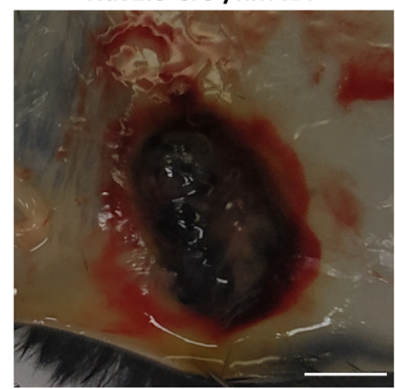

Fig. 1 Chemogenetic inhibition of neuronal activity in sensory Nav1.8 + nerve fibers triggers melanoma growth. A Schematic diagram of the Nav1.8-Cre +/hM4Di + experimental mouse model. Cre recombinase directs the expression of hM4Di specifically to sensory neurons in those mice. After the administration of CNO to those mice, neuronal activity in sensory neurons is inhibited. B Tumor-infiltrating sensory neurons are targeted in Nav1.8-Cre mice. $1 \times 10^{5}$ B16F10 melanoma cells were subcutaneously injected into Nav1.8-Cre/TdTomato mice, and tumor tissues were surgically removed 16 days later. Representative image of a Nav1.8-Cre/TdTomato mouse tumoral section with sensory nerve fibers infiltrating the tumor labelled with TdTomato fluorescence (red) and nuclei with DAPI (blue). C Capsaicin-induced spontaneous behavior test corroborates chemogenetic inhibition of sensory Nav1.8 + nerve fibers in Nav1.8-Cre ${ }^{+} / \mathrm{hM} \mathrm{Di}^{+}$mice after CNO treatment. Column charts show the licking

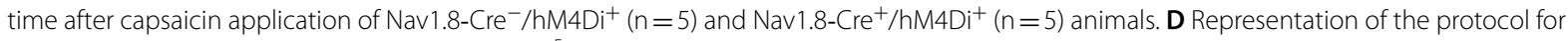
subcutaneous allograft melanoma growth. $1 \times 10^{5}$ B16F10 melanoma cells were subcutaneously injected into Nav1.8-Cre $/ \mathrm{hM} 4 \mathrm{Di}+(\mathrm{n}=5)$ and Nav1.8-Cre +/hM4Di + $(n=5)$ mice, followed by tumors removal for analysis after 16 days. CNO was daily intra-peritoneal injected at $1 \mathrm{mg} / \mathrm{kg}$. E Development curve of tumor growth from Nav1.8-Cre $/ \mathrm{hM} 3 \mathrm{Dq}^{+}$and Nav1.8-Cre $/ \mathrm{hM}^{-} \mathrm{Dq}^{+}$. Tumor volumes were assessed over time with a caliper. F Representative macroscopic image of B16F10 melanoma after dissection, left panel (Nav1.8-Cre $\left./ \mathrm{hM}_{4} \mathrm{Di}^{+}\right)$and right panel (Nav1.8-Cre ${ }^{+}$/ $\mathrm{hM} \mathrm{Di}^{+}$). G Tumor weight. (Nav1.8-Cre $/ \mathrm{hM}^{-} \mathrm{Di}^{+}: 0.50 \pm 0.04 \mathrm{~g}$; Nav1.8-Cre ${ }^{+} / \mathrm{hM} \mathrm{Di}^{+}: 0.98 \pm 0.23 \mathrm{~g}$ ). Data are shown as mean $\pm \mathrm{SEM}$. Unpaired t test (ns $P>0.05$; ${ }^{*} P<0.05$; ${ }^{* *} P<0.01$ ) 
intra-tumoral blood vessels' area (from $0.02 \pm 0.00$ to $0.03 \pm 0.01 \mu \mathrm{m}^{2}$ ) (Fig. 2A, B). Expression of Ki67 is used to determine the proliferation rate of malignant cancer cells [139], which is also associated with melanoma aggressiveness [76]. We found that genetic silencing of sensory innervations led to an increase in the proliferation rate within the melanoma (from $2074 \pm 55.32$ to $2454 \pm 168.4 \mathrm{Ki} 67+$ cells per $\mu^{2}$ ) (Fig. $2 \mathrm{C}$, D). We also observed after inhibition of sensory neurons firing a decrease in tumor-infiltrating CD4 $+\mathrm{T}$ cells (from $4.47 \times 10^{7} \pm 1.15 \times 10^{7}$ to $1.73 \times 10^{7} \pm 7.92 \times 10^{6}$ cells per mg of tumor) (Fig. 2E), in special, in IL-17-producing CD4 $+\mathrm{T}$ cells (from $1.63 \times 10^{7} \pm 1.30 \times 10^{6}$ to $3.77 \times 10^{6} \pm 3.27 \times 10^{6}$ cells per mg of tumor) (Fig. $2 \mathrm{~F}$ ),
A Nav1.8-Cre ${ }^{-} / \mathrm{hM}^{-} \mathrm{Di}^{+}$

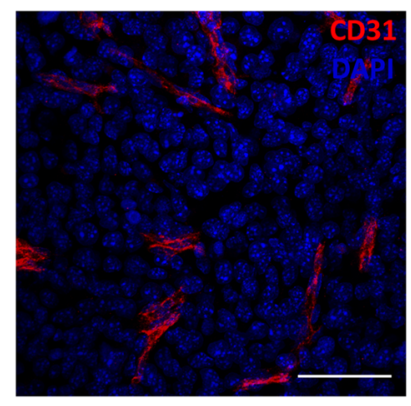

C

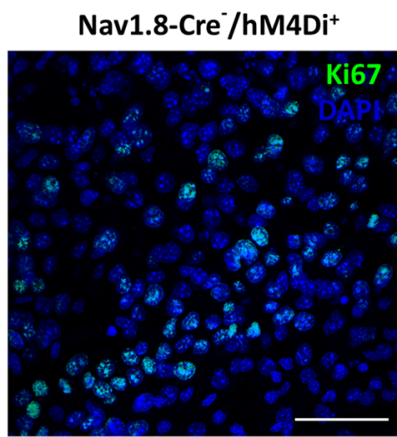

E

$\square$ Nav1.8-Cre $/ \mathrm{hM}^{-} \mathrm{Di}^{+}$
$\square$ Nav1.8-Cre $/ \mathrm{hM}^{+} \mathrm{Di}^{+}$

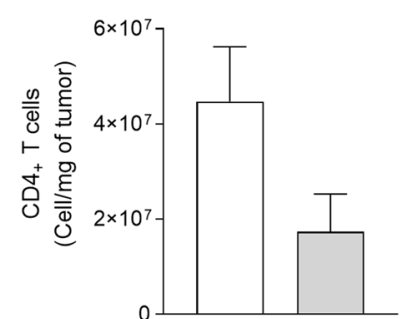

Nav1.8-Cre ${ }^{+} / \mathrm{hM} \mathrm{Di}^{+}$

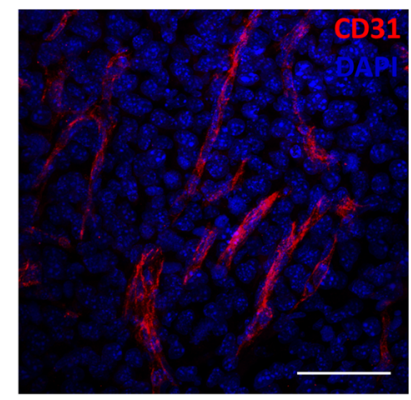

B
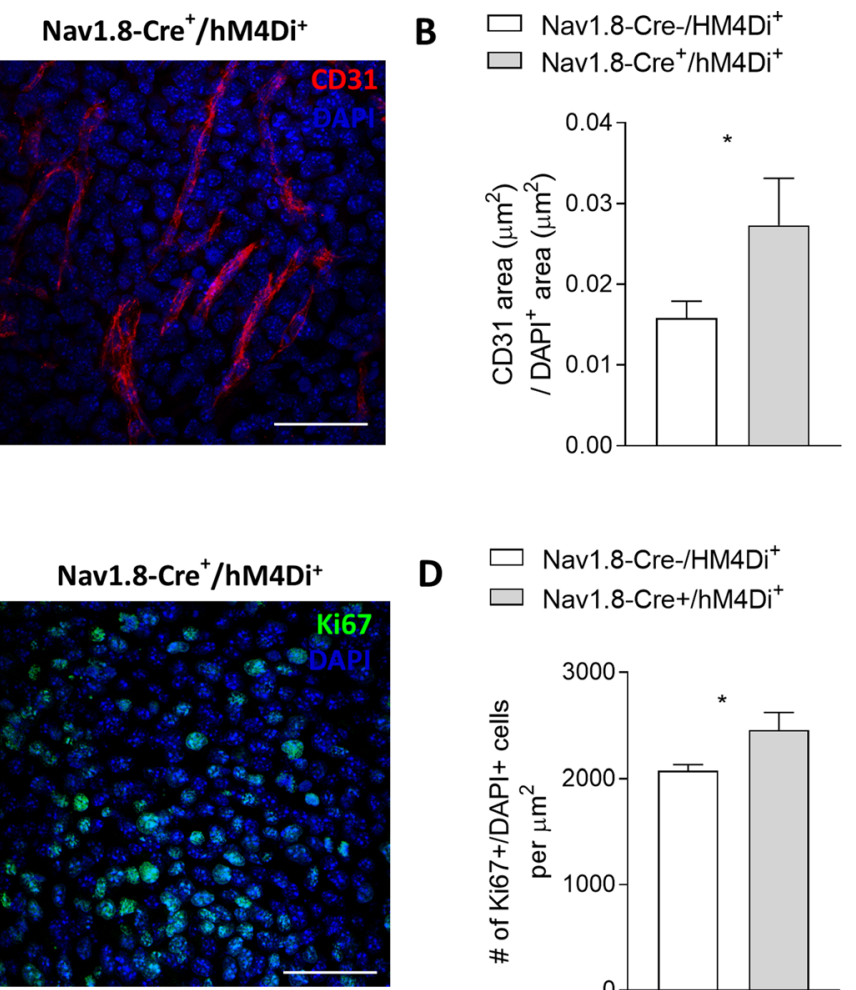

F

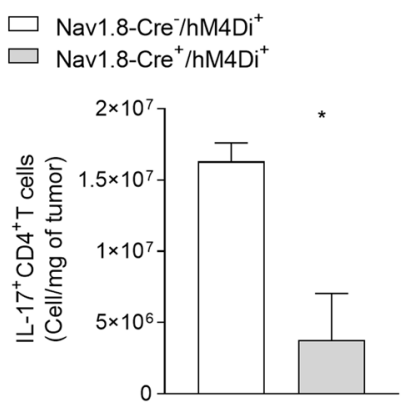

D $\square$ Nav1.8-Cre-/HM4Di ${ }^{+}$

$\square$ Nav1.8-Cre+/hM4Di ${ }^{+}$

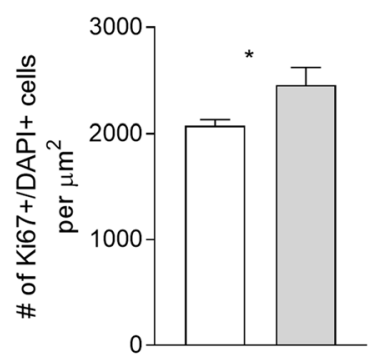

G

$\square$ Nav1.8-Cre $/ \mathrm{hM}^{-} \mathrm{Di}^{+}$

$\square$ Nav1.8-Cre ${ }^{+} / \mathrm{hM}^{-} \mathrm{Di}^{+}$

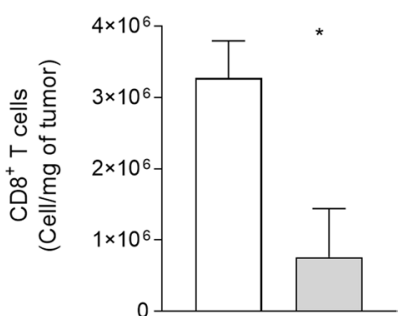

Fig. 2 Chemogenetic inhibition of neuronal activity in sensory Nav1.8 + innervations increases intra-tumoral proliferation and angiogenesis, and blocks anti-tumoral immune response. $1 \times 10^{5}$ B16F10 melanoma cells were subcutaneously injected into Nav1.8-Cre $/ \mathrm{hM} 4 \mathrm{Di}+(\mathrm{n}=5)$ and Nav1.8-Cre + /hM4Di + $(n=5)$ mice, followed by tumors removal for analysis after 16 days. A Representative immunofluorescence images of tumors labelled for endothelial cells (CD31; red) to identify blood vessels and nuclei (DAPl; blue). B Quantification of angiogenesis in melanomas by blood vessel area. C Representative immunofluorescence images of tumors labelled for Ki67 (Ki67; green) to identify cell proliferation and nuclei (DAPI; blue). D Quantification of proliferation in melanomas by the counting of Ki67 + cells per $\mu \mathrm{m}^{2}$. Absolute number of CD4 $+\mathbf{E}$ and CD8 $+\mathbf{G} T$ cells from the melanomas of B16F10-inoculated mice. F Graph shows absolute numbers of CD4 +T cells producers of IL-17. IL-17 levels were measured in cells isolated from tumors of B16F10-inoculated Nav1.8-Cre $/ \mathrm{hM}_{4} \mathrm{Di}^{+}$and Nav1.8-Cre $/ \mathrm{hM}^{+} \mathrm{Di} \mathrm{i}^{+}$animals. Data are shown as mean \pm SEM. Unpaired t test (ns $\left.P>0.05 ;{ }^{*} P<0.05\right)$ 
and a decrease in melanoma-infiltrating CD8 $+\mathrm{T}$ cells (from $3.27 \times 10^{6} \pm 5.22 \times 10^{5}$ to $7.62 \times 10^{5} \pm 6.78 \times 10^{5}$ cells per mg of tumor) (Fig. 2G). Our results indicate that inhibition of neuronal activity in sensory neurons promotes melanoma tumor advancement.

\section{Chemogenetic activation of hM3Dq excitatory DREADD receptors in Nav1.8 + neurons promotes melanoma regression}

As we found that inhibition of sensory neuron activity promotes melanoma advancement, we hypothesized that increasing sensory excitability would result in the reverse: blockage of melanoma progression. To test this hypothesis, we used again chemogenetics, by which we induced the expression of excitatory hM3Dq DREADDs [127] only in Nav1.8 + sensory neurons. We crossed Nav1.8-Cre mice to a mouse line with a Cre-dependent evolved Gq protein-coupled receptor (hM3Dq) expression. In the resulting Nav1.8-Cre +/hM3Dq + mice, upon removal of loxP-stop-loxP cassette by Cre recombination, the Gq-coupled hM3Dq is expressed specifically in Nav1.8-sensory nerve fibers. Sensory neurons in those mice can thus be overactivated by the administration of CNO. It has been shown previously that GqDREADD activation by CNO increases neuronal activity in the targeted neurons, including sensory neurons [68, 90], without changing the number of neurons [110]. To evaluate the role of sensory stimulation on tumor growth, we transplanted subcutaneously B16F10 melanoma cells to the lower right flank of both stimulatory DREADD-expressing mice (Nav1.8-Cre +/hM3Dq +) and their controls (Nav1.8-Cre-/hM3Dq + ). Following the cancer cell implantation, we treated mice daily with $\mathrm{CNO}$ to induce Nav1.8+ sensory neuron activation (controls were also treated with CNO) (Fig. 3A, B). After repeated sensory neuron activation, melanoma development was decreased in the sensory neuron-overactivated mice when compared to the controls (at day 14 , tumor volume per body weight was reduced from $3.51 \pm 0.89$ to $0.71 \pm 0.20 \mathrm{~mm}^{3}$; at day 16 , tumor weight was reduced from $0.38 \pm 0.07$ to $0.17 \pm 0.03$ g; Fig. $3 C-F$ ). Animal weights were not affected by genetic stimulation of sensory neurons in melanoma-bearing mice (data not shown). Moreover, genetic overactivation of sensory neurons led to a decrease in proliferating cells within the tumor (from $3050 \pm 203$ to $1292 \pm 367 \mathrm{Ki} 67+$ cells per $\mu \mathrm{m}^{2}$, analyzed by immunohistochemistry) (Fig. 3G, H), corroborated by flow cytometry analysis of CD45- cells for Ki67 expression (the was a decrease from $8.13 \pm 1.00$ to $5.07 \pm 0.70 \%$ of $\mathrm{CD} 45-/ \mathrm{Ki} 67+$ cells within the population of CD45- cells) (Fig. 3I). Additionally, there was a decrease in the intra-tumoral blood vessels' area (from $0.010 \pm 0.001$ to $0.006 \pm 0.001 \mu \mathrm{m}^{2}$ of CD31 + area $/ \mu \mathrm{m}^{2}$ of tumor area) (Fig. 3J, K). Our data suggest that increase in neuronal activity in sensory neurons counteracts melanoma development.

\section{Increase in sensory neuron activty affects melanoma immunosurveillance}

Functional studies in combination with histological analysis have demonstrated that tumor-infiltrating immune cells modulate melanoma cells' behavior, altering cancer outcomes $[38,72,79,83,112,130,133,134,152$, 153]. Given that sensory neurons may influence immune responses in non-cancer contexts, we sought to probe whether sensory neurons stimulation alters immune surveillance within the tumor.

Accumulating evidence has demonstrated that tumorinfiltrating neutrophils and PMN-MDSCs promote tumor development and progression $[21,39,65,107$, $136,138,140,150]$. Thus, we evaluated whether these cells are affected by sensory neurons' overactivation. We found that the number of melanoma-infiltrating neutrophils and PMN-MDSCs was significantly decreased in the sensory neuron-overactivated mice (Nav1.8$\mathrm{Cre}+/ \mathrm{hM} 3 \mathrm{Dq}+$ ) when compared to the controls (Nav1.8-Cre-/hM3Dq +) (from $12.02 \times 10^{7} \pm 3.45 \times 10^{7}$ to $4.69 \times 10^{7} \pm 7.10 \times 10^{6} \quad$ PMN-MDSCs per $\mathrm{mg}$ of tumor; and from $10.45 \times 10^{7} \pm 3.70 \times 10^{7}$ to $2.78 \times 10^{7} \pm 5.65 \times 10^{6}$ neutrophils per $\mathrm{mg}$ of tumor)

(See figure on next page.)

Fig. 3 Overstimulation of sensory Nav1.8 + nerve fibers decreases melanoma growth. A Schematic diagram of the Nav1.8-Cre+ /

$\mathrm{hM} 3 \mathrm{Dq}+$ experimental mouse model. Cre recombinase directs the expression of hM3Dq specifically to sensory neurons in those mice. After the administration of $\mathrm{CNO}$ to those mice, neuronal activity in sensory neurons is overactivated. B Representation of the protocol for subcutaneous allograft melanoma growth. $1 \times 10^{5}$ B16F10 melanoma cells were subcutaneously injected into Nav1.8-Cre ${ }^{-} / \mathrm{hM} 3 \mathrm{Dq}+(\mathrm{n}=14)$ and Nav1.8$\mathrm{Cre}+/ \mathrm{hM} 3 \mathrm{Dq}+(\mathrm{n}=13)$ mice, and tumors were removed for analysis after 16 days. CNO was injected daily intra-peritoneally at $1 \mathrm{mg} / \mathrm{kg}$. C Development curve of tumor growth from Nav1.8-Cre $/ \mathrm{hM} \mathrm{Dq}^{+}$and $\mathrm{Nav1.8-Cre} / \mathrm{hM} \mathrm{Dq}^{+}$. Tumor volumes were assessed over time with a caliper. D Representative macroscopic images of B16F10 melanoma tumors after dissection, left panel $\left(\mathrm{Nav} 1.8-\mathrm{Cre}^{-} / \mathrm{hM} \mathrm{DDq}{ }^{+}\right)$and right panel (Nav1.8-Cre ${ }^{+} / \mathrm{hM} \mathrm{Dq}{ }^{+}$). E Tumor weight. (Nav1.8-Cre $/ \mathrm{hM3Dq}^{+}: 0.38 \pm 0.07 ; \mathrm{Nav1.8-Cre} / \mathrm{hM}^{-} \mathrm{Dq}^{+}: 0.17 \pm 0.03$ ). F Tumor weight corrected by animal body weight. G Representative immunofluorescence images of tumors labelled for Ki67 (Ki67; green) to identify cell proliferation and nuclei

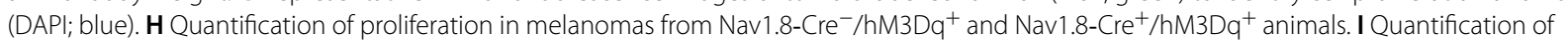
proliferation (Ki67+) by flow cytometry in CD45- cells from tumors of Nav1.8-Cre $-/ \mathrm{hM} 3 \mathrm{Dq}^{+}$and Nav1.8-Cre ${ }^{+} / \mathrm{hM} \mathrm{DDq}{ }^{+}$mice. J Representative immunofluorescence images of tumor sections labelled for endothelial cells (CD31; red) to identify blood vessels and nuclei (DAPI; blue). $\mathbf{K}$ Quantification of angiogenesis in melanomas by blood vessel area. Data are shown as mean \pm SEM. Unpaired test $\left(\mathrm{ns} P>0.05 ;{ }^{*} P<0.05\right.$; $\left.{ }^{* *} P<0.01\right)$ 


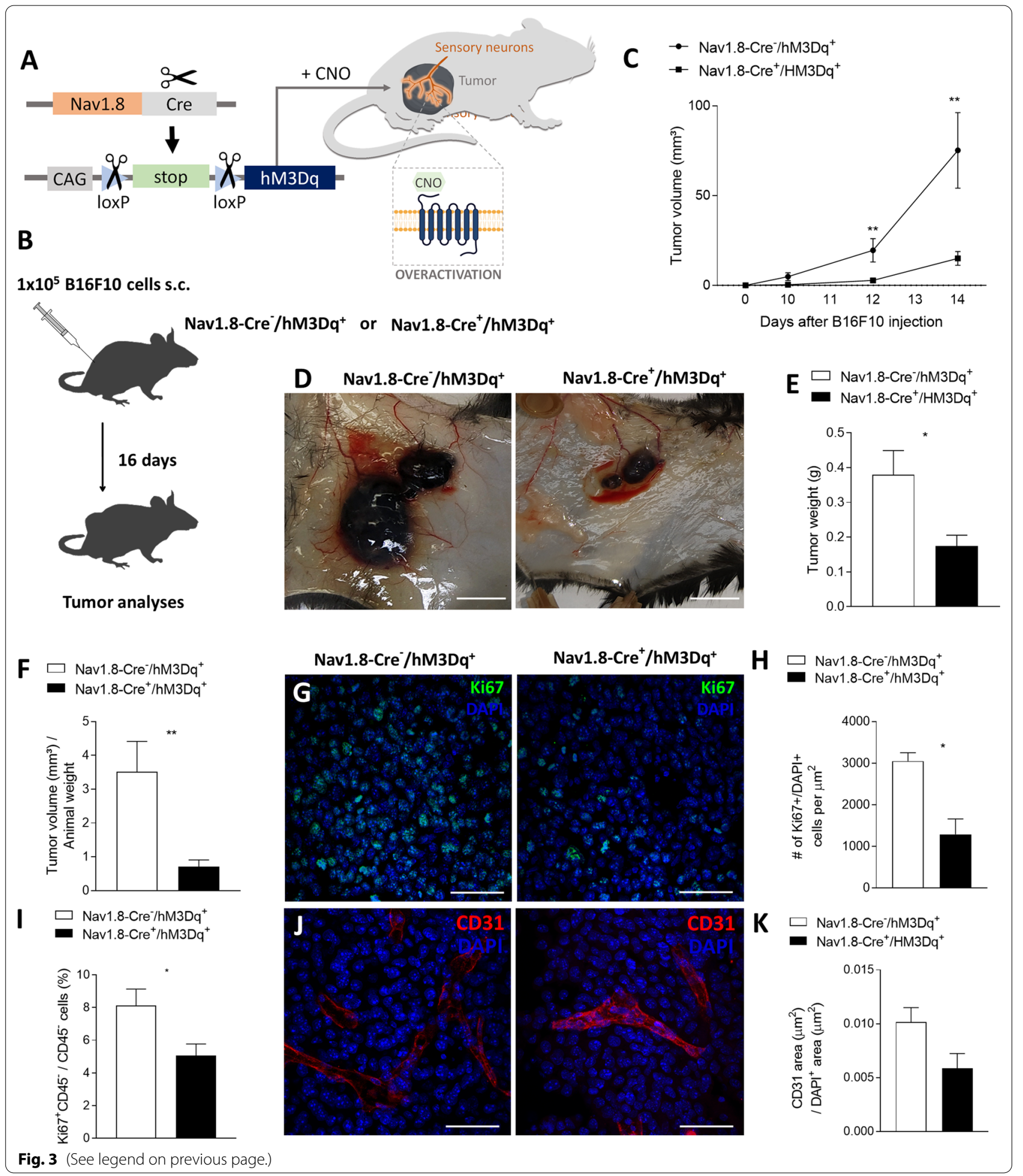

(Fig. 4A, B). On the other hand, we found that the number of tumor-infiltrating dendritic cells, which counteract the proliferation of melanoma cells [137], was significantly increased (from $5.53 \times 10^{7} \pm 8.80 \times 10^{6}$ to
$1.07 \times 10^{8} \pm 2.27 \times 10^{7}$ dendritic cells per mg of tumor) (Fig. 4C).

Recent breakthroughs in cancer immunotherapy have revealed the remarkable ability of the immune system 

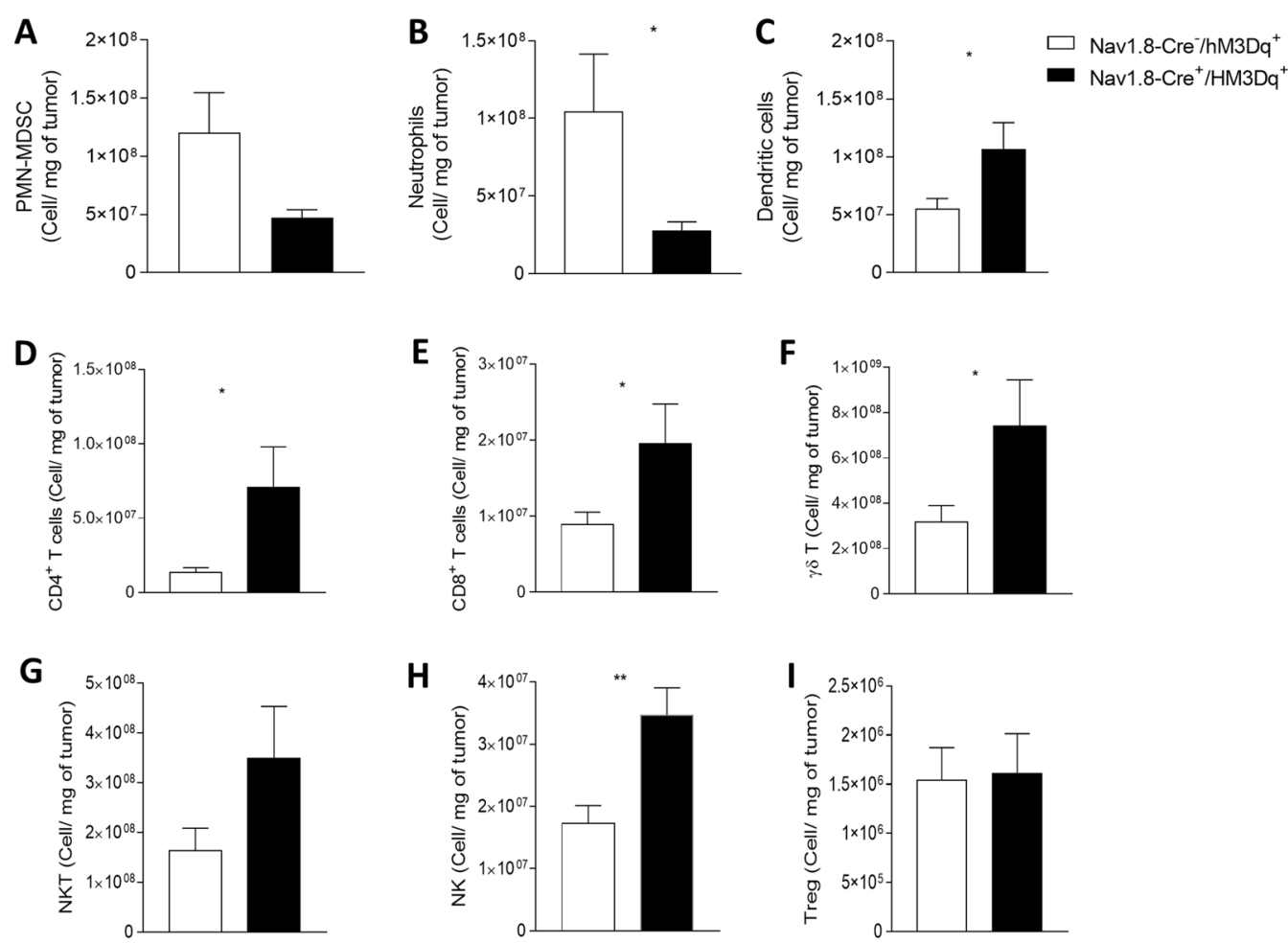

Fig. 4 Sensory neurons overactivation improves anti-tumor immunity by decreasing tumor-infiltrating immunosuppressive cells, increasing dendritic cells and by promoting CD4 $+\mathrm{T}, \mathrm{CD} 8+\mathrm{T}, \gamma \delta \mathrm{T}$, NKT, and NK-cell infiltration. Immune cells from B16F10-inoculated mice were analyzed ex vivo in Nav1.8-Cre $-/ \mathrm{hM} \mathrm{Dq}^{+}(\mathrm{n}=14)$ and Nav1.8-Cre $/ \mathrm{hM} \mathrm{Dq}^{+}(\mathrm{n}=13)$ mice. Column charts show the proportion of PMN/MDSC (A) Neutrophils (B) and Dendritic cells (C) quantified in the tumor microenvironment. (D-I) TIL from B16F10-inoculated Nav1.8-Cre-/hM3Dq + $(n=14)$ and Nav1.8-Cre +/hM3Dq + $(n=13)$ mice were analyzed ex vivo. Absolute number of CD4 +T cells $(\mathbf{D}), C D 8+T$ cells $(\mathbf{E}), \gamma \delta T$ cells $(\mathbf{F})$, NKT cells (G), NK cells $(\mathbf{H})$, and Treg cells (I) from the melanomas of B16F10-inoculated mice. Data are shown as mean $\pm \mathrm{SEM}$, Unpaired $\mathrm{t}$ test, ${ }^{*} .01<P<.05$; ${ }^{* *} .001<P<.01$

to fight different types of cancers, including melanoma. The phenotypes and numbers of prevalent tumor-infiltrating lymphocytes are predictive of response to immunotherapy and key modulators of disease progression. Thus, we examined how tumor-infiltrating lymphocytes are affected by sensory neurons' overstimulation. We detected an increase in tumor-infiltrating $\mathrm{CD} 4+\mathrm{T}$ cells (from $2.91 \times 10^{6} \pm 1.04 \times 10^{6}$ to $1.09 \times 10^{7} \pm 2.92 \times 10^{6}$ cells per $\mathrm{mg}$ of tumor), $\mathrm{CD} 8+\mathrm{T}$ cells (from $8.94 \times 10^{6} \pm 1.60 \times 10^{6}$ to $1.96 \times 10^{7} \pm 5.20 \times 10^{6}$ cells per $\mathrm{mg}$ of tumor), $\gamma \delta \mathrm{T}$ cells (from $31.76 \times 10^{7} \pm 7.32 \times 10^{7}$ to $74.14 \times 10^{7} \pm 20.40 \times 10^{7}$ cells per $\mathrm{mg}$ of tumor), NKT cells (from $16.34 \times 10^{7} \pm 4.6 \times 10^{7}$ to $34.92 \times 10^{7} \pm 10.42 \times 10^{7}$ cells per mg of tumor) and NK cells (from $1.72 \times 10^{7} \pm 2.90 \times 10^{6}$ to $3.47 \times 10^{7} \pm 4.40 \times 10^{6}$ cells per mg of tumor) (Fig. $4 \mathrm{D}-$ $\mathrm{H})$, while regulatory $\mathrm{T}$ cells, which mediate immunosuppression in the tumor microenvironment [66], were not altered (Fig. 4I). Immune checkpoint molecules, such as cytotoxic $\mathrm{T}$ lymphocyte antigen 4 (CTLA-4) and programmed cell death 1 (PD-1), act fine-tuning the intense immune responses that might kill healthy cells $[27,55,122]$. Their expression in cytotoxic $\mathrm{T}$ cells may lead to dysfunction of these cells, affecting their effector function $[11,146]$. We found that increase in the firing of sensory neurons prevented the increase of immune checkpoint markers of tumor infiltrating CD8 $+\mathrm{T}$ cells and CD4+ T cells (Fig. 5 and Additional file 1: Figure 1). The percentage of CTLA-4-expressing CD4+tumorinfiltrating lymphocytes decreased from $29.43 \pm 4.04 \%$ in Nav1.8-Cre ${ }^{-} / \mathrm{hM}_{3} \mathrm{Dq}^{+}$to $19.08 \pm 2.80 \%$ in Nav1.8-Cre ${ }^{+} /$ $\mathrm{hM} \mathrm{Dq}^{+}$animals (Fig. 5A); similarly, the percentage of PD-1-expressing CD4+tumor-infiltrating lymphocytes decreased from $15.02 \pm 2.62 \%$ in Nav1.8-Cre ${ }^{-} / \mathrm{hM}^{-} \mathrm{Dq}^{+}$ to $7.85 \pm 1.43 \%$ in Nav1.8-Cre ${ }^{+} / \mathrm{hM} \mathrm{Dq}^{+}$mice (Fig. $5 \mathrm{~B}$, C). The percentage of PD-1-expressing CD8+tumorinfiltrating cytotoxic lymphocytes also decreased from $22,03 \pm 2,66 \%$ in Nav1.8-Cre ${ }^{-} / \mathrm{hM}^{2} \mathrm{Dq}^{+}$to $12.99 \pm 3.85 \%$ in Nav1.8-Cre ${ }^{+} / \mathrm{hM} \mathrm{Dq}^{+}$animals (Fig. 5E), while the expression of CTLA-4 did not vary in these cells (Fig. 5D, F). In addition, no differences were found in CTLA-4 and PD-1 expression on $\gamma \delta$ T cells (Fig. 5G, H and I), NKT 

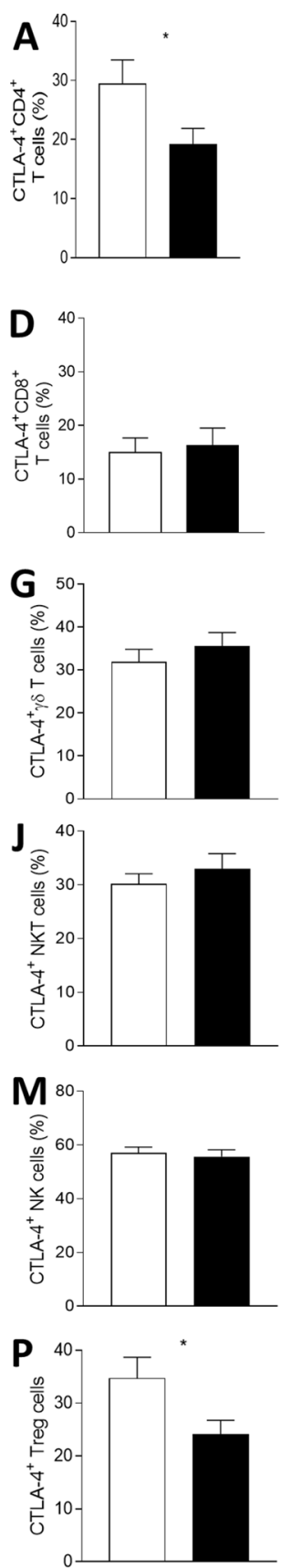
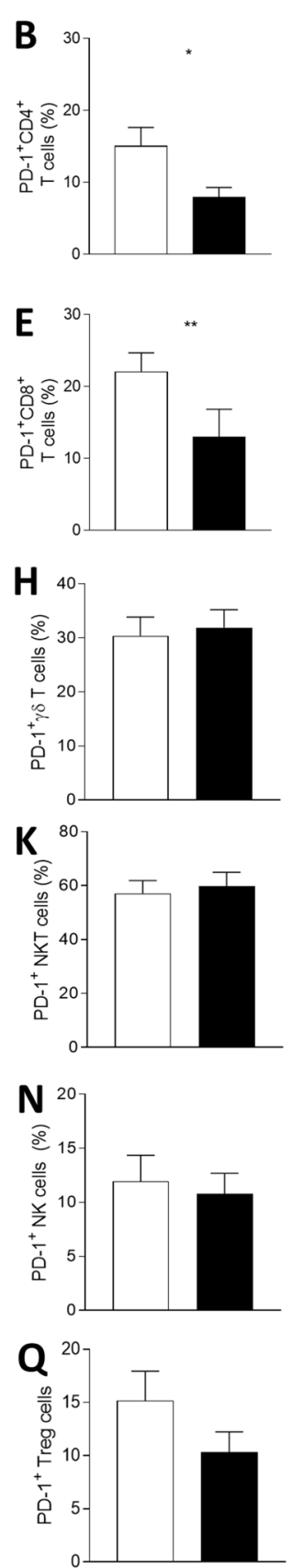
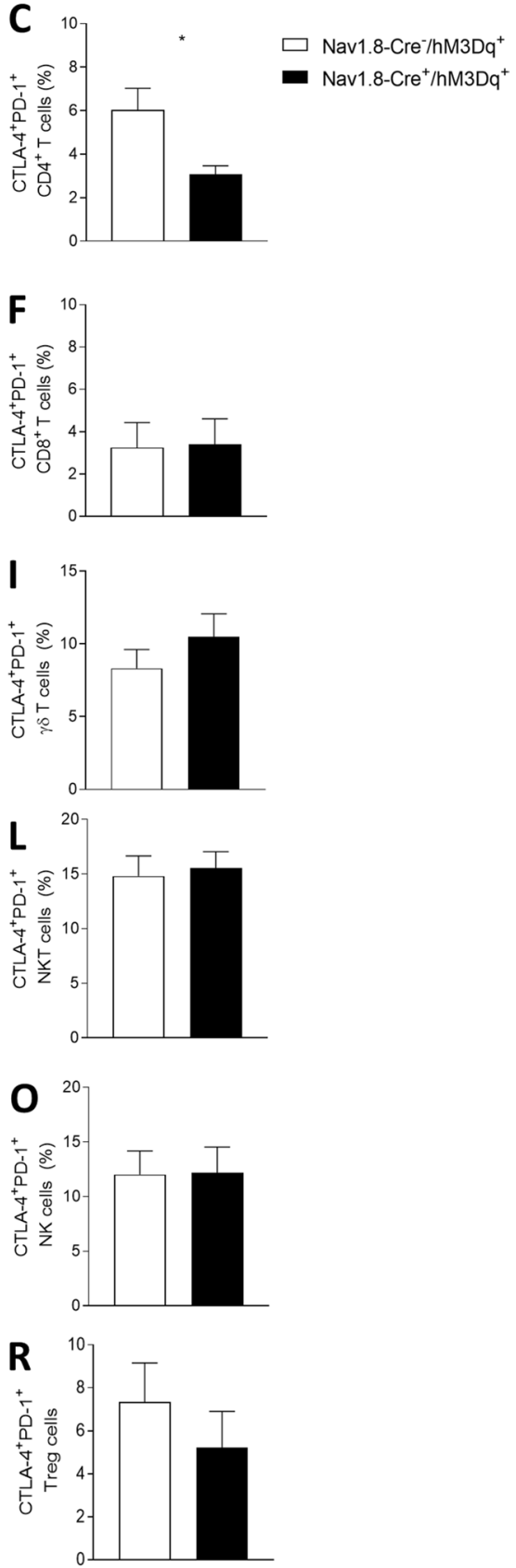

Fig. 5 Sensory neurons overstimulation prevent the increase of immune checkpoint markers in tumor infiltrating CD8 $+T$ cells and CD4+T cells. Immune cells from tumors of B16F10-inoculated mice were analyzed ex vivo in Nav1.8-Cre ${ }^{-} / \mathrm{hM} \mathrm{DDq}{ }^{+}(\mathrm{n}=14)$ and Nav1.8-Cre ${ }^{+} / \mathrm{hM}^{\mathrm{DDq}} \mathbf{q}^{+}(\mathrm{n}=13)$ mice. Column charts show proportion of CTLA-4 (A, D, G, J, M, P), PD-1 (B, E, H, K, N, Q) and CTLA-4/PD-1 co-expressing (C, F, I, L, O, R) CD4+T cells $(\mathbf{A}, \mathbf{B}, \mathbf{C}), \mathbf{C D} 8+\mathrm{T}$ cells $(\mathbf{D}, \mathbf{E}, \mathbf{F}), \gamma \delta$ T cells $(\mathbf{G}, \mathbf{H}, \mathbf{I})$, NKT cells $(\mathbf{J}, \mathbf{K}, \mathbf{L})$, NK cells $(\mathbf{M}, \mathbf{N}, \mathbf{O})$, and Treg cells $(\mathrm{P}, \mathbf{Q}, \mathrm{R})$ from tumors of B16F10-inoculated mice. Data are shown as mean $\pm \mathrm{SEM}$, Unpaired $\mathrm{t}$ test, ${ }^{*} .01<P<.05 ;{ }^{* *} .001<P<.01$

cells (Fig. 5J, K and L), NK cells (Fig. 5M, N and O) and Treg cells (Fig. 5P, Q and R). Overall, our data suggest that sensory neurons overactivation induces improvement of $\mathrm{T}$ cells effector functions within the tumor microenvironment.
It has been reported that $\mathrm{CD} 4+$ and $\mathrm{CD} 8+$ lymphocytes secreting IL-17 promote melanoma regression [91, 99]. Here, we detected in response to sensory neuron firing an increase in melanoma-infiltrating IL-17-producing $\mathrm{CD} 4+\mathrm{T}$ cells (from $2.45 \times 10^{7} \pm 6.05 \times 10^{6}$ to 
$30.78 \times 10^{7} \pm 9.20 \times 10^{7}$ cells per mg of tumor) as well as in melanoma-infiltrating IL-17-producing CD8 $+\mathrm{T}$ cells (from $5.02 \times 10^{7} \pm 0.90 \times 10^{7}$ to $20.08 \times 10^{7} \pm 5.92 \times 10^{7}$ cells per mg of tumor) (Fig. 6A). In parallel, we did not detect significant changes in the number of other tumor-infiltrating lymphocytes producing IL-17 or in IFN- $\gamma$-producing lymphocytes after sensory neuron overactivation (Fig. 6B). Altogether, our results suggest that sensory neurons induce a Th17-immune response in the melanoma microenvironment.

Lymph nodes are an integral part of the adaptive immune system in our organism and are essential for the effective immune responses. Melanoma draining lymph nodes are influenced by the primary tumor, but may also prime the immune suppressive microenvironment, playing critical roles in promoting cancer immune escape [33, $86,87,97]$. It is completely unknown whether sensory neuron overactivation may affect the immune cells also within the tumor draining lymph nodes. Herein, we analyzed immune cells from tumor draining and non-tumordraining lymph nodes from CNO-treated stimulatory DREADD-expressing animals (Nav1.8-Cre $+/ \mathrm{hM} 3 \mathrm{Dq}+)$ and their controls (Nav1.8-Cre-/hM3Dq + ). Tumor draining and non-tumor-draining lymph nodes were isolated from the ipsilateral and contralateral side, respectively, of the implanted melanoma (Fig. 7A, B). We found that the effect of sensory neurons stimulation in tumor draining lymph nodes mimics the immune response within the primary tumor, but not in non-tumor-draining lymph nodes. In the tumor-draining lymph nodes, we found an increase in the number of CD8 + cytotoxic $\mathrm{T}$ cells after sensory neurons' overstimulation (from
$13.68 \times 10^{7} \pm 3.50 \times 10^{7}$ to $22.41 \times 10^{7} \pm 2.17 \times 10^{7}$ cells per mg of tumor) (Fig. 7C); while we did not detect any differences in the numbers of $\mathrm{T}$ cells in the tumor nondraining lymph nodes (Fig. 7D). These data indicate a possible priming effect of tumor on the adjacent draining lymph nodes.

We also observed, in the tumor-draining lymph nodes, increases in IFN- $\gamma$-producing $\mathrm{CD} 4+\mathrm{T}$ cells (from $8.31 \times 10^{6} \pm 2.65 \times 10^{6}$ to $1.94 \times 10^{7} \pm 3.36 \times 10^{6}$ cells per lymph node), in IFN- $\gamma$-producing NK cells (from $4.72 \times 10^{4} \pm 2.46 \times 10^{4}$ to $8.44 \times 10^{5} \pm 2.22 \times 10^{5}$ cells per lymph node), in IL-17-producing CD8 $+\mathrm{T}$ cells (from $2.38 \times 10^{6} \pm 4.27 \times 10^{5}$ to $1.28 \times 10^{7} \pm 1.64 \times 10^{6}$ cells per lymph node), and in IL-17-producing NKT cells (from $2.35 \times 10^{6} \pm 5.19 \times 10^{5}$ to $4.08 \times 10^{6} \pm 5.00 \times 10^{5}$ cells per lymph node) after sensory neurons CNO-stimulation (Fig. 7E). In tumor non-draining lymph nodes, we detected increases in IFN- $\gamma$-producing $\mathrm{CD} 4+\mathrm{T}$ cells (from $5.73 \times 10^{6} \pm 2.17 \times 10^{6}$ to $1.92 \times 10^{7} \pm 4.23 \times 10^{6}$ cells per lymph node), in IFN- $\gamma$-producing CD8 + T cells (from $1.36 \times 10^{7} \pm 5.57 \times 10^{6}$ to $4.77 \times 10^{7} \pm 1.19 \times 10^{7}$ cells per lymph node), in IFN- $\gamma$-producing $\gamma \delta \mathrm{T}$ cells (from $2.27 \times 10^{6} \pm 8.55 \times 10^{5}$ to $1.03 \times 10^{7} \pm 3.22 \times 10^{6}$ cells per lymph node), and in IFN- $\gamma$-producing NK cells (from $1.80 \times 10^{5} \pm 4.70 \times 10^{4}$ to $6.82 \times 10^{5} \pm 1.53 \times 10^{5}$ cells per lymph node) (Fig. 7F). Our data suggest that lymphocytes at the lymph nodes may be contributing to the response against the melanoma observed within the tumor microenvironment after sensory neurons' overactivation as both, IFN- $\gamma$ and IL-17, may contribute to enhance the anti-tumoral response in the context of melanoma [92, 109, 151]. Altogether, our data suggest that
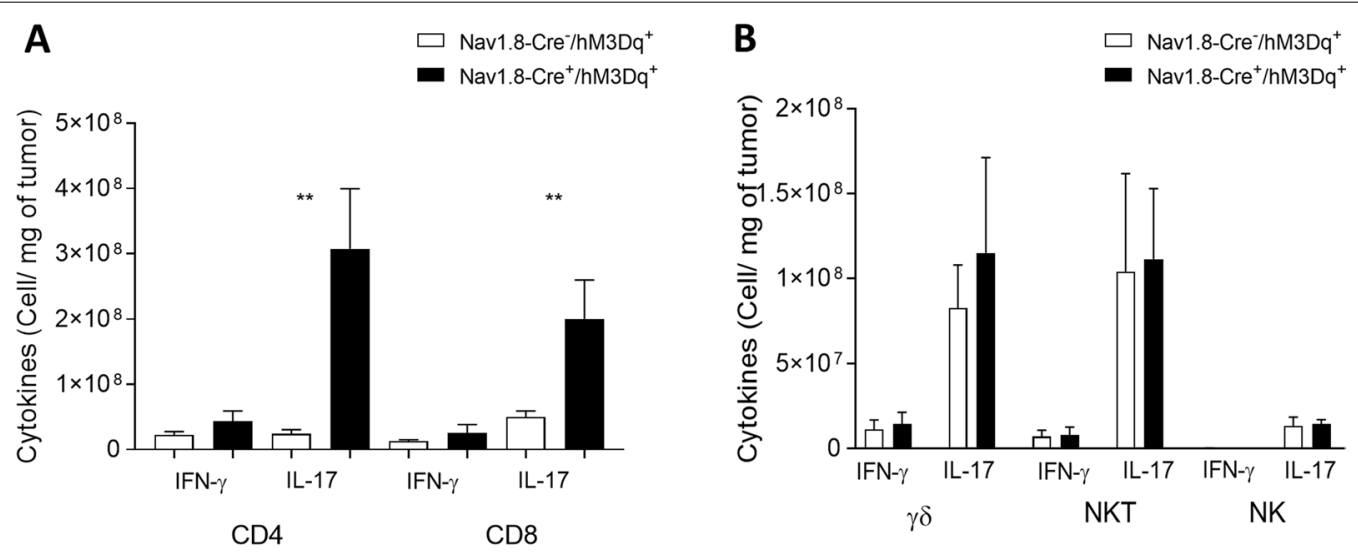

Fig. 6 Sensory neurons overactivation promote an increase in tumor-infiltrating IL-17-producing CD4 + and CD8+T cells. TIL from melanomas of B16F10-inoculated Nav1.8-Cre-/hM3Dq $+(n=14)$ and Nav1.8-Cre +/hM3Dq + $(n=13)$ mice were analyzed. TIL from B16F10-inoculated mice were analyzed after $4 \mathrm{~h}$ of culture. A Column charts show absolute numbers of CD4+ and CD8 +T cells producers of IFN- $\gamma$ and IL-17. B Column charts show absolute number of $\gamma \delta$ T cells, NKT cells and NK cells producing IFN- $\gamma$ and IL-17. Cytokines levels were measured in cells isolated from tumors of B16F10-inoculated Nav1.8-Cre ${ }^{-} / \mathrm{hM}^{2} \mathrm{Dq}^{+}$and Nav1.8-Cre ${ }^{+} / \mathrm{hM} \mathrm{Dq}^{+}$mice. Data are shown as mean \pm SEM, Unpaired t test, ${ }^{*} .01<P<.05$; **.001<P<.01 
A

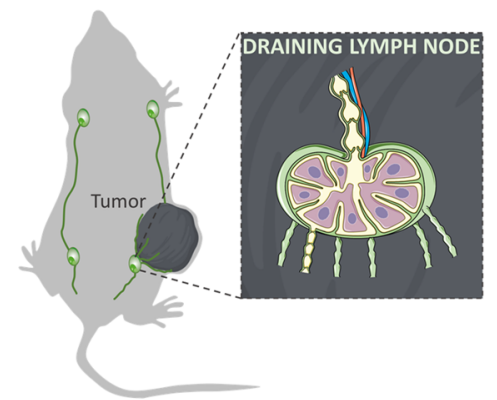

C $\square$ Nav1.8-Cre//hM3Dq ${ }^{+}$

- Nav1.8-Cre ${ }^{+} / \mathrm{hM}^{2} \mathrm{Dq}^{+}$

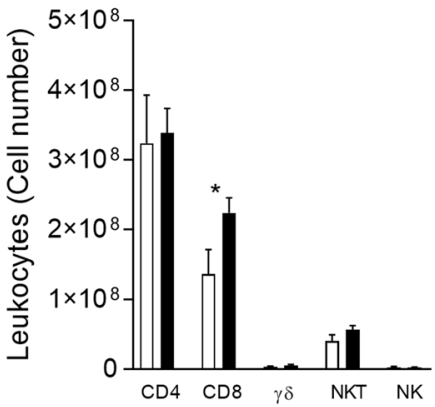

E $\square$ Nav1.8-Cre $-/ \mathrm{hM}^{-} \mathrm{Dq}^{+}$

- Nav1.8-Cre ${ }^{+} / \mathrm{hM} \mathrm{Dq}^{+}$

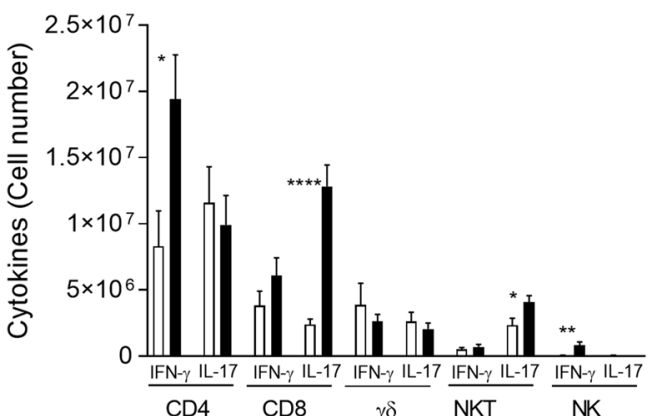

B

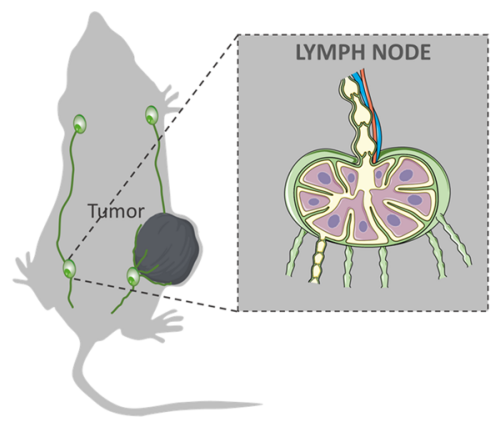

D
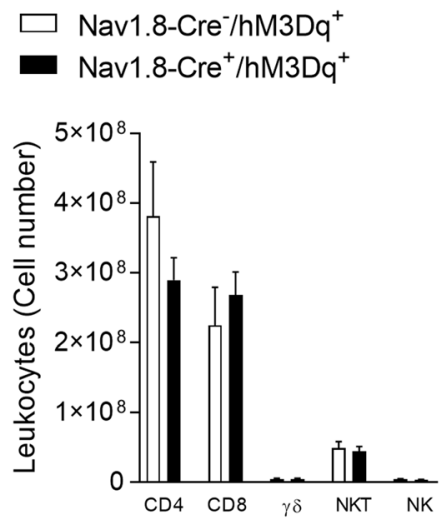

$\mathbf{F}$

Nav1.8-Cre $/ \mathrm{hM}^{-} \mathrm{Dq}^{+}$

Nav1.8-Cre ${ }^{+} / \mathrm{hM}^{3} \mathrm{Dq}^{+}$

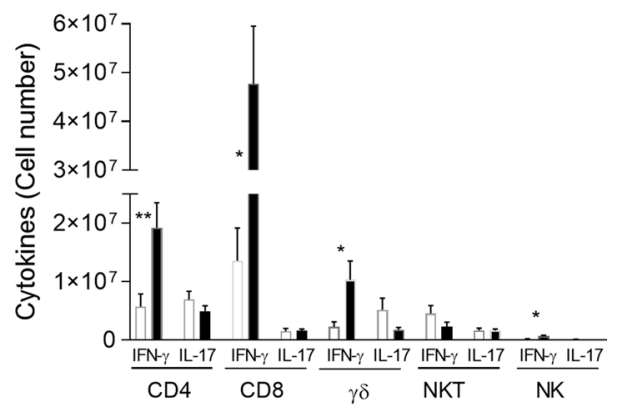

Fig. 7 Tumor-draining lymph nodes present an increase in effector CD8+T-cells after overstimulation of sensory neurons, while the number of lymphocytes in tumor non-draining lymph nodes doesnt change. A Schematic representation of the collected tumor draining lymph nodes. TIL from tumor-draining lymph nodes of B16F10-inoculated Nav1.8-Cre-/hM3Dq $+(n=14)$ and Nav1.8-Cre $+/ h M 3 D q+(n=13)$ mice were analyzed. B Absolute number of CD4+, CD8 $+, \gamma \delta, N K T$ and NK cells from tumor-draining lymph nodes of B16F10-inoculated mice. C IFN- $\gamma$ and IL-17 were quantified in CD4, CD8, $\gamma \delta, N K T$ and NK cells. D Schematic representation of the collected tumor non-draining lymph nodes. TIL from tumor non-draining lymph nodes of B16F10-inoculated Nav1.8-Cre-/hM3Dq $+(n=14)$ and Nav1.8-Cre $+/ h M 3 D q+(n=13)$ mice were analyzed. E Absolute number of $\mathrm{CD} 4+, \mathrm{CD} 8+, \gamma \delta, \mathrm{NKT}$ and NK cells from tumor non-draining lymph nodes of B16F10-inoculated mice. F IFN- $\gamma$ and IL-17 were quantified in $\mathrm{CD} 4, \mathrm{CD} 8, \gamma \delta, \mathrm{NKT}$ and NK cells. Cytokines levels were measured in cells from tumor-draining and tumor non-draining lymph nodes of B16F10-inoculated Nav1.8-Cre-/hM3Dq + and Nav1.8-Cre +/hM3Dq + mice. Data are shown as mean \pm SEM. Unpaired t test, ${ }^{*} .01<P<.05$; ${ }^{* *} .001<P<.01 ;{ }^{* * *} P<0.0001$

sensory neurons stimulation alters immune surveillance that impacts melanoma development.
High expression of genes related to sensory neurons correlates with best prognosis in human melanoma patients

In order to investigate our findings also in human tumors, we analyzed The Cancer Genome Atlas (TCGA) samples 
from 103 Skin Cutaneous Melanoma (SKCM) patients. First, we stratified SKCM patients in two groups, alive or dead based on a 5-year interval. Next, we searched for differentially expressed genes between these groups. We found 112 up-regulated and 195 down-regulated genes ( $\mid \log 2$ (Fold change) $\mid \geq 1$ and adjusted $P$-value $<0.05$; Table 2). Next, we performed a Gene Ontology (GO) analysis of Biological Processes (BP) in which these genes are involved. The up-regulated genes set are enriched for three biological processes, while the down-regulated genes are enriched to a wide variety of processes (Fig. 8A; Table 3). Curiously, for the former (up-regulated genes), two out of three Biological Processes represent nervous system development (Fig. 8A), indicating the importance of neuronal networks in melanoma outcomes. Next, we investigated the interactions (and putative regulation) of 34 sensory neurons-related genes which were selected from the literature $[29,37,51,114,141,144]$ (Table 4). Figure $8 \mathrm{~B}$ shows a strong connection among 18 of these genes, suggesting that they work on the same cellular pathways or cell types. Next, using the expression levels of these 34 gene markers for sensory neurons we investigated their potential to be "a signature" associated with SKCM cancer patient survival. Figure $8 \mathrm{C}$ shows that high expression of these genes (lower patient scores) are associated with a better overall survival of SKCM patients. Next, we investigated the expression of these genes in the two sample sets (alive and dead patients). We found that SCN10A, which codifies Nav1.8, a key gene based on which our mouse models target sensory neurons, is more expressed in alive than in dead patients (Fig. 8D). Finally, we investigated the impact of SCN10A expression on SKCM patients' survival. Figure $8 \mathrm{E}$ shows that high expression of SCN10A trends to be associated with a better overall survival of SKCM patients, even without statistical support $(P$-value $=0.26)$. Taken together, these results confirm that genes related to nervous system development are enriched in samples from live Skin Cutaneous Melanoma patients. Focusing on gene markers for sensory neurons, we confirmed that these genes are strongly connected, suggesting a synergistic activity, and that the higher expression of some of these genes are associated with a higher overall survival. Strikingly, the high expression of SCN10A is potentially associated with better SKCM patient survival, indicating that the presence of sensory neurons within melanoma counteracts cancer progression. We also found that TCGA samples from tumors with a worst prognosis (dead patients) have an enrichment of genes promoting angiogenesis (Tables 3 and 5; 15 genes related to angiogenesis). We focused on this set of 15 genes related to angiogenesis and we confirmed that they are strongly connected (Additional file 2: Fig. 2), indicating a synergic function in promoting angiogenesis. Our results indicate that there is an increase in genes related with angiogenesis in tumors with worst prognosis (from dead patients) and a decrease in their expression in tumors from alive patients which show an increased expression of SCN10a, a sensory neuron marker used in this study (Fig. 8C, D, E). By using the CIBERSORT tool [102], we investigated immune infiltrated cells in the same TCGA cohort of alive vs. dead patients (Additional file 3: Fig. 3). CIBERSORT uses gene expression (RNA sequencing data) and support vector regression combined with prior knowledge of expression profiles from purified leukocyte subsets (gene signatures) to produce an estimation of the abundances of immune infiltrated cells subpopulations in a sample. In line with our data presented in this manuscript, we have checked the enrichment of immune infiltrated cells in the tumors of patients alive vs. dead (Additional file 4: Fig. 4). We found an increase of $\mathrm{CD} 4+\mathrm{T}$ cells, CD8 $+\mathrm{T}$ cells, NK cells and dendritic cells in patients with better prognosis (alive). Thus, tumors showing a better prognosis (alive) have an increased infiltration of some key immune cells. Additionally, microarray data evidenced a down-regulation of genes related to the Th17 immune response in melanoma patients (Additional file 5: Fig. 5). These analyses are consistent with the data generated in our mouse models: that the overactivation of sensory innervations in the tumor microenvironment was associated with suppressed melanoma progression. Albeit gene expression in tumor biopsies from human cancer patients is used as a tool to define novel biomarkers and to contribute to prognosis, the obtained data should be also validated by the quantification of sensory neuron-related proteins in human melanoma biopsies and correlation with clinical outcomes in future research.

\section{Discussion}

In the present study, we examined how melanoma progression is affected by sensory neurons activity. Our chemogenetic approach revealed that inhibition of sensory activity promotes tumor growth and intra-tumoral angiogenesis. In contrast, excitation of sensory neurons induces melanoma regression with decrease in tumor growth and in new blood vessel formation, as well as a boost in the anti-tumor immune surveillance (Fig. 9). This work indicates that induction of hyperactivation in Nav1.8-expressing sensory neurons represents a potential new therapeutic path in the battle against melanoma.

Just as the role of particular genes in a specific biological process can be examined by evaluating examining the assessable consequences that result from their removal (e.g. using knockout animals), the role of neurons in the tumor microenvironment was previously assessed by eliminating them [32, 36, 71, 89, 108, 115, 
Table 2 Analyzes of genes from The Cancer Genome Atlas (TCGA) samples from 103 Skin Cutaneous Melanoma (SKCM) patients

\begin{tabular}{|c|c|c|}
\hline \\
\hline \multicolumn{3}{|c|}{$\begin{array}{l}\text { Up-regulated genes in alive } x \text { dead } \\
\text { Gene.symbol } \quad \log 2 \mathrm{FC}\end{array}$} \\
\hline SLC5A4 & 4.35910 & $1.29 E-09$ \\
\hline VGF & 3.26059 & $1.06 \mathrm{E}-05$ \\
\hline NPPC & 3.24826 & $2.60 \mathrm{E}-05$ \\
\hline LINC00698 & 3.76414 & $3.90 E-05$ \\
\hline SPACA3 & 2.67598 & 1.97E-04 \\
\hline$V C X 3 A$ & 4.95714 & $2.21 \mathrm{E}-04$ \\
\hline PRSS56 & 4.13411 & $4.15 E-04$ \\
\hline ARHGAP8 & 2.75929 & 4.15E-04 \\
\hline VCX & 3.63292 & $4.28 \mathrm{E}-04$ \\
\hline LINC01287 & 3.48781 & $7.19 E-04$ \\
\hline NGFR & 1.99998 & 1.16E-03 \\
\hline NAT16 & 2.23940 & 1.16E-03 \\
\hline RP13-143G15.4 & 2.26728 & $1.34 \mathrm{E}-03$ \\
\hline HLA-J & 1.67323 & $1.38 \mathrm{E}-03$ \\
\hline LHFPL4 & 2.80159 & $2.04 \mathrm{E}-03$ \\
\hline RP11-376N17.4 & 2.27953 & $2.10 E-03$ \\
\hline ZNF689 & 1.08305 & $2.33 \mathrm{E}-03$ \\
\hline DCD & 6.91950 & $2.44 \mathrm{E}-03$ \\
\hline SLITRK5 & 2.38821 & $2.59 E-03$ \\
\hline ARPP21 & 3.84108 & $2.67 \mathrm{E}-03$ \\
\hline TFAP2B & 2.73943 & $2.68 \mathrm{E}-03$ \\
\hline VIT & 2.42509 & $3.21 \mathrm{E}-03$ \\
\hline HPCAL4 & 1.70772 & $3.30 E-03$ \\
\hline LINC00645 & 3.54408 & $4.05 E-03$ \\
\hline KLHL32 & 1.29974 & $4.09 E-03$ \\
\hline TRIML2 & 2.32595 & $4.80 E-03$ \\
\hline GFAP & 2.04141 & $5.21 \mathrm{E}-03$ \\
\hline MYOZ2 & 2.33172 & $6.94 E-03$ \\
\hline PPY & 2.13167 & $7.88 \mathrm{E}-03$ \\
\hline ARX & 2.02055 & $8.39 E-03$ \\
\hline LRRTM2 & 1.75474 & $8.39 E-03$ \\
\hline C20orf203 & 1.95612 & $8.43 E-03$ \\
\hline LSMEM2 & 1.36377 & $8.43 \mathrm{E}-03$ \\
\hline NRTN & 1.74226 & $8.58 \mathrm{E}-03$ \\
\hline RP11-809C18.3 & 1.54681 & $8.89 E-03$ \\
\hline FOSB & 1.08702 & $9.10 \mathrm{E}-03$ \\
\hline PASD1 & 4.57206 & $9.14 E-03$ \\
\hline UNC93B3 & 1.84987 & $9.14 \mathrm{E}-03$ \\
\hline RP11-469H8.6 & 2.35144 & $9.14 \mathrm{E}-03$ \\
\hline $\mathrm{BCO} 1$ & 2.17260 & $9.80 E-03$ \\
\hline XKR7 & 1.93913 & $1.00 E-02$ \\
\hline $\mathrm{RDH} 5$ & 1.36688 & $1.04 \mathrm{E}-02$ \\
\hline PAGE1 & 3.69526 & $1.10 \mathrm{E}-02$ \\
\hline RP5-907D15.4 & 1.73346 & 1.10E-02 \\
\hline AC003092.1 & 2.31155 & $1.20 E-02$ \\
\hline IGHV1-58 & 3.09550 & $1.20 \mathrm{E}-02$ \\
\hline FOXG1 & 3.56195 & $1.36 \mathrm{E}-02$ \\
\hline GBA3 & 2.67229 & $1.39 \mathrm{E}-02$ \\
\hline
\end{tabular}

Table 2 (continued)

Up-regulated genes in alive $x$ dead

\begin{tabular}{|c|c|c|}
\hline Gene.symbol & $\log 2 \mathrm{FC}$ & FDR \\
\hline FKSG51 & 1.89383 & $1.39 E-02$ \\
\hline BTNL8 & 1.87248 & $1.46 \mathrm{E}-02$ \\
\hline ACHE & 1.18749 & $1.52 \mathrm{E}-02$ \\
\hline KCNJ11 & 1.81031 & $1.52 \mathrm{E}-02$ \\
\hline ENPP7P2 & 1.45231 & $1.64 \mathrm{E}-02$ \\
\hline CCKBR & 2.06353 & $1.73 E-02$ \\
\hline PCSK1N & 1.87835 & $1.76 \mathrm{E}-02$ \\
\hline RP11-114G11.5 & 3.04186 & 1.76E-02 \\
\hline EFTUD1P1 & 2.13819 & 1.77E-02 \\
\hline OR2N1P & 5.08848 & $1.83 \mathrm{E}-02$ \\
\hline CA10 & 2.21148 & $1.85 \mathrm{E}-02$ \\
\hline SLCO5A1 & 1.55420 & $1.88 \mathrm{E}-02$ \\
\hline IBSP & 1.81676 & $1.91 \mathrm{E}-02$ \\
\hline RP11-88|21.1 & 3.56590 & $1.94 \mathrm{E}-02$ \\
\hline MAGEA9 & 5.10603 & 1.96E -02 \\
\hline SYP & 1.03514 & $2.15 \mathrm{E}-02$ \\
\hline NBEAP1 & 1.55393 & $2.17 \mathrm{E}-02$ \\
\hline RP5-965G21.4 & 1.14894 & $2.18 \mathrm{E}-02$ \\
\hline MPZ & 1.95997 & $2.22 \mathrm{E}-02$ \\
\hline RP11-299H22.3 & 2.95030 & $2.22 \mathrm{E}-02$ \\
\hline $\mathrm{FBXO2}$ & 1.34819 & $2.31 E-02$ \\
\hline LGSN & 2.41190 & $2.31 E-02$ \\
\hline $\mathrm{RDH} 8$ & 2.14341 & $2.32 \mathrm{E}-02$ \\
\hline AC073325.2 & 1.90901 & $2.38 \mathrm{E}-02$ \\
\hline GAGE1 & 3.92238 & $2.44 \mathrm{E}-02$ \\
\hline MYB & 1.16344 & $2.55 \mathrm{E}-02$ \\
\hline AATK & 1.23997 & $2.63 E-02$ \\
\hline DOK7 & 1.48390 & $2.72 \mathrm{E}-02$ \\
\hline AC068580.7 & 2.45353 & $2.72 \mathrm{E}-02$ \\
\hline RP11-36D19.9 & 1.99056 & $2.72 E-02$ \\
\hline HAPLN2 & 1.51979 & $2.73 E-02$ \\
\hline TDRD12 & 2.24362 & $3.09 \mathrm{E}-02$ \\
\hline RP11-159H10.3 & 1.83751 & $3.18 \mathrm{E}-02$ \\
\hline CHGB & 1.62908 & $3.32 \mathrm{E}-02$ \\
\hline RCN3 & 1.25033 & $3.32 \mathrm{E}-02$ \\
\hline RP5-1171110.5 & 1.33948 & $3.32 \mathrm{E}-02$ \\
\hline NMRK2 & 2.15724 & 3.33E-02 \\
\hline TNNI3 & 1.50305 & 3.33E-02 \\
\hline DPEP3 & 1.81124 & $3.33 E-02$ \\
\hline DPYSL5 & 2.12990 & $3.33 E-02$ \\
\hline ZNF365 & 1.53513 & $3.39 E-02$ \\
\hline KCNQ2 & 1.98246 & $3.51 E-02$ \\
\hline PMP2 & 2.27248 & $3.53 \mathrm{E}-02$ \\
\hline HAVCR1 & 2.17185 & $3.56 \mathrm{E}-02$ \\
\hline RP1-140K8.5 & 1.72113 & $3.72 E-02$ \\
\hline ROR1-AS1 & 2.03339 & $3.75 E-02$ \\
\hline C1QTNF1-AS1 & 1.82857 & $3.75 \mathrm{E}-02$ \\
\hline WFDC1 & 1.74139 & $3.99 E-02$ \\
\hline DEFB126 & 2.40171 & $3.99 E-02$ \\
\hline LBP & 1.71514 & $3.99 \mathrm{E}-02$ \\
\hline
\end{tabular}


Table 2 (continued)

\begin{tabular}{|c|c|c|}
\hline \multicolumn{3}{|c|}{ Up-regulated genes in alive $x$ dead } \\
\hline Gene.symbol & $\log 2 \mathrm{FC}$ & FDR \\
\hline $\mathrm{CDH} 12$ & 2.39970 & $3.99 \mathrm{E}-02$ \\
\hline FABP7 & 2.11963 & $3.99 \mathrm{E}-02$ \\
\hline MAGEB17 & 1.73309 & $3.99 \mathrm{E}-02$ \\
\hline MYBPC1 & 1.99301 & $3.99 \mathrm{E}-02$ \\
\hline RP4-764D2.1 & 1.03824 & 4.07E-02 \\
\hline CD5L & 1.77129 & $4.21 E-02$ \\
\hline CPN2 & 1.81842 & $4.21 \mathrm{E}-02$ \\
\hline ZNF727 & 2.20430 & $4.21 \mathrm{E}-02$ \\
\hline CST1 & 2.16587 & 4.35E-02 \\
\hline RP11-9G1.3 & 2.55148 & $4.48 \mathrm{E}-02$ \\
\hline LL22NC03-22D1.1 & 2.49624 & $4.50 \mathrm{E}-02$ \\
\hline NPFFR1 & 1.60868 & 4.54E-02 \\
\hline MYRIP & 1.45769 & 4.67E-02 \\
\hline RP11-369C8.1 & 2.61906 & 4.95E-02 \\
\hline \multicolumn{3}{|c|}{ Down-regulated genes in alive $x$ dead } \\
\hline Gene.symbol & $\log 2 \mathrm{FC}$ & FDR \\
\hline AVPR1A & -2.06066 & 8.65E-08 \\
\hline KRT16P2 & -5.08308 & $1.00 \mathrm{E}-07$ \\
\hline CHST8 & -3.38063 & 7.38E-07 \\
\hline ST6GAL2 & -2.68516 & 1.74E-06 \\
\hline PRSS35 & -3.35395 & $4.71 \mathrm{E}-6$ \\
\hline SLC8A3 & -3.18840 & $9.15 E-06$ \\
\hline CRABP1 & -3.07142 & $9.15 \mathrm{E}-6$ \\
\hline HSPB3 & -3.24770 & $2.15 \mathrm{E}-05$ \\
\hline TREX2 & -2.43750 & $2.15 E-05$ \\
\hline B3GNT4 & -1.77784 & $2.26 \mathrm{E}-05$ \\
\hline NPTX1 & -2.61603 & 1.97E-04 \\
\hline PI3 & -3.60121 & 2.70E-04 \\
\hline HEYL & -1.67534 & $2.99 \mathrm{E}-04$ \\
\hline ID3 & -1.32736 & $3.67 \mathrm{E}-04$ \\
\hline ADRB2 & -1.81924 & $3.91 \mathrm{E}-04$ \\
\hline C6orf223 & -2.13191 & 4.15E-04 \\
\hline AJAP1 & -1.92423 & 4.56E-04 \\
\hline CHRNA4 & -2.95110 & 4.69E-04 \\
\hline RHCG & -2.92093 & $5.10 \mathrm{E}-04$ \\
\hline PART1 & -2.14901 & $5.50 \mathrm{E}-04$ \\
\hline ALOX12 & -1.23574 & $5.50 E-04$ \\
\hline RSPO3 & -1.48997 & $6.13 E-04$ \\
\hline GDPD3 & -1.74062 & 7.38E-04 \\
\hline CNFN & -2.84236 & 8.27E-04 \\
\hline ANO2 & -1.70534 & $9.14 \mathrm{E}-04$ \\
\hline OVOL1 & -2.75293 & $9.14 \mathrm{E}-04$ \\
\hline CLDN4 & -2.52180 & $9.56 \mathrm{E}-04$ \\
\hline GREB1L & -2.35313 & $1.12 \mathrm{E}-03$ \\
\hline IGLV9-49 & -3.84182 & $1.20 E-03$ \\
\hline TGM1 & -2.85878 & $1.28 \mathrm{E}-03$ \\
\hline CYSRT1 & -2.10472 & $1.34 \mathrm{E}-03$ \\
\hline LYPD5 & -2.27815 & $1.34 \mathrm{E}-03$ \\
\hline
\end{tabular}

Table 2 (continued)

Down-regulated genes in alive $x$ dead

\begin{tabular}{|c|c|c|}
\hline Gene.symbol & $\log 2 \mathrm{FC}$ & FDR \\
\hline CYP19A1 & -1.84923 & $1.41 \mathrm{E}-03$ \\
\hline ACTG2 & -1.63424 & $1.41 \mathrm{E}-03$ \\
\hline ABCG4 & -2.12582 & $1.41 \mathrm{E}-03$ \\
\hline FAM3D & -2.12972 & $1.41 \mathrm{E}-03$ \\
\hline PAPSS2 & -1.50601 & $1.41 \mathrm{E}-03$ \\
\hline AC124789.1 & -2.07689 & $1.41 \mathrm{E}-03$ \\
\hline RASL11B & -1.54784 & $1.43 \mathrm{E}-03$ \\
\hline MAFB & -1.28214 & 1.43E-03 \\
\hline NGF & -1.73693 & $2.01 E-03$ \\
\hline AC006116.20 & -1.47472 & $2.01 E-03$ \\
\hline $\mathrm{S} 100 \mathrm{~A} 12$ & -2.64402 & 2.10E-03 \\
\hline KLK14 & -2.29647 & $2.39 \mathrm{E}-03$ \\
\hline IL1RN & -2.09008 & $2.39 \mathrm{E}-03$ \\
\hline B3GNT8 & -1.78028 & $2.39 E-03$ \\
\hline GPX3 & -1.88978 & $2.39 E-03$ \\
\hline CDA & -2.22423 & $2.68 \mathrm{E}-03$ \\
\hline $\mathrm{CHN1}$ & -1.30585 & $2.68 \mathrm{E}-03$ \\
\hline PRSS27 & -1.71351 & $2.68 \mathrm{E}-03$ \\
\hline TMEM79 & -1.43989 & $3.52 \mathrm{E}-03$ \\
\hline NOTCH3 & -1.22297 & 3.57E-03 \\
\hline FGF18 & -1.35348 & $3.80 E-03$ \\
\hline HGF & -1.19158 & $4.28 \mathrm{E}-03$ \\
\hline SH3RF3 & -1.23159 & 4.49E-03 \\
\hline KRT37 & -4.22818 & 4.57E-03 \\
\hline SH3RF3-AS1 & -1.31293 & 4.57E-03 \\
\hline ZC3H12A & -1.35742 & 4.67E-03 \\
\hline TBX4 & -1.84817 & $5.39 \mathrm{E}-03$ \\
\hline CLIC3 & -2.31078 & $5.58 \mathrm{E}-03$ \\
\hline LRRC43 & -1.34142 & $5.69 E-03$ \\
\hline NRARP & -1.42862 & 5.97E-03 \\
\hline B3GNT3 & -1.97566 & $6.61 E-03$ \\
\hline ELF3 & -2.07351 & $6.73 E-03$ \\
\hline LRRN2 & -1.90993 & $6.80 E-03$ \\
\hline NFE4 & -2.76901 & $6.80 E-03$ \\
\hline KLK10 & -2.72909 & $6.94 \mathrm{E}-03$ \\
\hline LINC01121 & -1.68460 & $7.15 E-03$ \\
\hline SDCBP2 & -1.48707 & $7.88 \mathrm{E}-03$ \\
\hline FAM83G & -1.50253 & $7.88 \mathrm{E}-03$ \\
\hline ZNF469 & -1.06394 & $7.88 \mathrm{E}-03$ \\
\hline ROR2 & -1.15831 & $7.92 \mathrm{E}-03$ \\
\hline LYNX1 & -1.75074 & 7.92E-03 \\
\hline VSIG10L & -1.54237 & $7.92 \mathrm{E}-03$ \\
\hline PITX1 & -2.50531 & $8.39 E-03$ \\
\hline PNMA5 & -2.07397 & $8.43 E-03$ \\
\hline LTB4R2 & -1.38009 & $8.43 E-03$ \\
\hline GTSF1 & -1.95376 & $8.89 E-03$ \\
\hline RP3-449H6.1 & -2.80745 & $8.89 E-03$ \\
\hline PKDCC & -1.47224 & $9.03 \mathrm{E}-03$ \\
\hline
\end{tabular}


Table 2 (continued)

\begin{tabular}{|c|c|c|}
\hline \multicolumn{3}{|c|}{ Down-regulated genes in alive $x$ dead } \\
\hline Gene.symbol & $\log 2 \mathrm{FC}$ & FDR \\
\hline C11orf87 & -1.86276 & $9.10 E-03$ \\
\hline C9orf47 & -1.63063 & $9.10 \mathrm{E}-03$ \\
\hline KLK12 & -3.43996 & $9.10 E-03$ \\
\hline SPNS2 & -1.33949 & $9.43 \mathrm{E}-03$ \\
\hline $\mathrm{TCHH}$ & -1.56077 & $1.00 \mathrm{E}-02$ \\
\hline ADRA1D & -1.45232 & $1.01 \mathrm{E}-02$ \\
\hline LINC00675 & -2.28274 & $1.01 E-02$ \\
\hline GLP1R & -1.93142 & $1.04 \mathrm{E}-02$ \\
\hline DLX5 & -1.61403 & $1.05 \mathrm{E}-02$ \\
\hline JUP & -1.81687 & $1.10 E-02$ \\
\hline GREM1 & -1.36182 & 1.10E-02 \\
\hline FUT2 & -1.65558 & 1.10E-02 \\
\hline FLJ43879 & -1.73000 & 1.10E-02 \\
\hline RP4-530115.9 & -1.37950 & 1.10E-02 \\
\hline ADAMTSL4 & -1.15965 & 1.10E-02 \\
\hline LGALS9C & -1.74166 & $1.11 \mathrm{E}-02$ \\
\hline RP11-145A3.1 & -1.28593 & 1.20E-02 \\
\hline LINC00689 & -2.44100 & 1.20E-02 \\
\hline RP11-57C13.6 & -2.13756 & 1.20E-02 \\
\hline WNT11 & -1.67218 & $1.23 E-02$ \\
\hline SOX11 & -1.31510 & 1.27E-02 \\
\hline SMCO2 & -1.86262 & $1.29 E-02$ \\
\hline AC104654.2 & -1.54674 & $1.34 \mathrm{E}-02$ \\
\hline RP11-715H19.2 & -2.54830 & 1.45E-02 \\
\hline MALL & -1.65870 & 1.50E-02 \\
\hline SLPI & -2.56276 & 1.50E-02 \\
\hline ZNF385B & -1.82165 & $1.52 \mathrm{E}-02$ \\
\hline AC079305.10 & -1.07337 & $1.52 \mathrm{E}-02$ \\
\hline RARRES1 & -1.09119 & $1.56 \mathrm{E}-02$ \\
\hline C1orf177 & -1.80915 & $1.64 \mathrm{E}-02$ \\
\hline MIR4635 & -1.81095 & $1.68 \mathrm{E}-02$ \\
\hline KCNK12 & -1.74033 & 1.70E-02 \\
\hline CTC-525D6.2 & -3.65998 & 1.75E-02 \\
\hline FGF7 & -1.14265 & $1.76 \mathrm{E}-02$ \\
\hline KRT82 & -2.71344 & $1.76 \mathrm{E}-02$ \\
\hline CTD-2554C21.3 & -2.09957 & 1.76E-02 \\
\hline VNN3 & -1.87348 & $1.94 \mathrm{E}-02$ \\
\hline KRT17P6 & -2.54509 & $1.94 \mathrm{E}-02$ \\
\hline CD36 & -1.47332 & $1.98 \mathrm{E}-02$ \\
\hline ALDH1L1 & -1.84277 & 1.99E-02 \\
\hline KRT25 & -3.64328 & $2.04 \mathrm{E}-02$ \\
\hline GLIS3 & -1.24797 & $2.06 \mathrm{E}-02$ \\
\hline SPRR2D & -2.65861 & $2.06 \mathrm{E}-02$ \\
\hline CEACAM19 & -1.48405 & $2.11 \mathrm{E}-02$ \\
\hline ZNF154 & -1.19730 & 2.12E-02 \\
\hline FBLIM1 & -1.00682 & $2.15 E-02$ \\
\hline OR7E11P & -3.14060 & $2.15 E-02$ \\
\hline ZNF185 & -1.43741 & $2.27 \mathrm{E}-02$ \\
\hline
\end{tabular}

Table 2 (continued)

\section{Down-regulated genes in alive $x$ dead}

\begin{tabular}{|c|c|c|}
\hline Gene.symbol & $\log 2 \mathrm{FC}$ & FDR \\
\hline MYOC & -3.14651 & 2.30E-02 \\
\hline SULT2B1 & -2.16618 & $2.31 \mathrm{E}-02$ \\
\hline PLA2G4E-AS1 & -1.49669 & $2.32 \mathrm{E}-02$ \\
\hline IL36G & -2.54342 & $2.39 \mathrm{E}-02$ \\
\hline IGKV2-29 & -3.58127 & $2.43 E-02$ \\
\hline PADI3 & -1.60173 & $2.45 \mathrm{E}-02$ \\
\hline WDR87 & -2.67496 & $2.45 \mathrm{E}-02$ \\
\hline CTD-2555C10.3 & -1.62741 & $2.45 E-02$ \\
\hline SMPD3 & -1.51129 & $2.63 \mathrm{E}-02$ \\
\hline LYPD3 & -2.15151 & $2.63 \mathrm{E}-02$ \\
\hline SPINK9 & -2.18753 & $2.63 \mathrm{E}-02$ \\
\hline RP3-405J10.2 & -1.89008 & $2.63 \mathrm{E}-02$ \\
\hline KRT17 & -2.51044 & $2.65 \mathrm{E}-02$ \\
\hline RP11-845M18.6 & -2.34005 & $2.70 E-02$ \\
\hline CPXM2 & -1.33321 & $2.72 \mathrm{E}-02$ \\
\hline GDPD2 & -1.69177 & $2.79 E-02$ \\
\hline LINC01482 & -1.58713 & $2.84 \mathrm{E}-02$ \\
\hline KRT8P13 & -2.24864 & 3.07E-02 \\
\hline ANGPT2 & -1.42767 & 3.23E-02 \\
\hline TMEM45B & -1.81149 & $3.23 \mathrm{E}-02$ \\
\hline KLK13 & -2.41601 & $3.23 E-02$ \\
\hline IGHE & -2.55145 & $3.23 E-02$ \\
\hline SPRR2A & -2.94281 & $3.23 E-02$ \\
\hline RP11-91J3.3 & -1.87453 & $3.23 \mathrm{E}-02$ \\
\hline CTC-490G23.2 & -2.34066 & $3.32 \mathrm{E}-02$ \\
\hline ADAMTS15 & -1.20759 & $3.33 \mathrm{E}-02$ \\
\hline $\mathrm{RHOD}$ & -1.51767 & $3.33 \mathrm{E}-02$ \\
\hline COL28A1 & -1.56903 & 3.33E-02 \\
\hline RP11-752L20.3 & -1.07531 & $3.33 E-02$ \\
\hline AC133785.1 & -2.02852 & 3.47E-02 \\
\hline GNA15 & -1.37100 & $3.49 \mathrm{E}-02$ \\
\hline FAM46B & -1.22702 & $3.53 \mathrm{E}-02$ \\
\hline KRT80 & -2.07094 & $3.53 \mathrm{E}-02$ \\
\hline TWIST2 & -1.11079 & $3.53 E-02$ \\
\hline KRT42P & -2.45179 & $3.53 \mathrm{E}-2$ \\
\hline PRSS50 & -1.86776 & $3.64 \mathrm{E}-02$ \\
\hline TBX1 & -1.26122 & $3.69 E-2$ \\
\hline KRT81 & -1.69382 & $3.75 E-02$ \\
\hline ALOX12B & -2.41352 & $3.75 E-02$ \\
\hline KCNMA1-AS1 & -1.82562 & $3.79 E-02$ \\
\hline HS3ST3A1 & -1.28721 & $3.84 \mathrm{E}-02$ \\
\hline USP2 & -1.07928 & $3.99 E-02$ \\
\hline BMP4 & -1.23615 & $3.99 E-02$ \\
\hline G6PC2 & -2.36196 & $3.99 E-02$ \\
\hline RP11-169K16.4 & -1.98903 & $3.99 E-02$ \\
\hline COL8A1 & -1.22669 & 4.09E-02 \\
\hline SIX2 & -1.02697 & 4.09E-02 \\
\hline KRT17P2 & -2.21877 & 4.21E-02 \\
\hline
\end{tabular}


Table 2 (continued)

\begin{tabular}{lcl}
\hline Down-regulated genes in alive $\boldsymbol{x}$ dead & \\
\hline Gene.symbol & log2FC & FDR \\
\hline BDKRB1 & -1.22248 & $4.35 E-02$ \\
LINC00857 & -1.30729 & $4.35 E-02$ \\
CREB3L1 & -1.04632 & $4.44 E-02$ \\
CCBE1 & -1.04094 & $4.44 E-02$ \\
PRR15L & -1.31182 & $4.48 E-02$ \\
ST8SIA2 & -1.70296 & $4.50 E-02$ \\
S100A9 & -2.02615 & $4.55 E-02$ \\
SIK1 & -1.83293 & $4.60 E-02$ \\
EPN3 & -1.93269 & $4.65 E-02$ \\
ZBTB16 & -1.22227 & $4.67 E-02$ \\
ATP8B5P & -1.00252 & $4.76 E-02$ \\
DUOX1 & -1.72011 & $4.78 E-02$ \\
TRIM53CP & -2.79481 & $4.80 E-02$ \\
C6Orf132 & -1.81165 & $4.87 E-02$ \\
MDFI & -1.36942 & $4.91 E-02$ \\
CATSPERB & -1.85527 & $4.94 E-02$ \\
HSPE1P5 & -1.47888 & $4.94 E-02$ \\
DEFB4A & -3.10613 & $4.95 E-02$ \\
SDC1 & -1.36628 & $4.96 E-02$ \\
\hline
\end{tabular}

116, 121, 154, 158]. Nevertheless, although biomedical research has gained some insights into the function of intra-tumoral neurons using loss-of-function studies with surgical or pharmacologic denervation, these strategies are mostly not specific to a given neuronal type. Importantly, a disadvantage of all these studies is that neuronal killing may generate secondary undesirable indirect side effects caused by the inflammatory tissular response which may influence the observed phenotypes (Männ et al. 2016; Christiaansen, Boggiatto, and Varga 2014; Bennett et al. 2005). To circumvent these limitations, novel powerful technologies have been created in the field of modern neuroscience which allow to manipulate the firing of specific neurons without killing them: optogenetics and chemogenetics. These methods use genetic strategies to deliver the expression of lightsensitive proteins or designer receptors exclusively activated by designer drugs, respectively, to the membrane of defined neuronal populations. Therefore, by using these techniques, manipulation of neurons by exposure to light or to designer drugs, without killing them, became feasible. As melanoma is a chronic disease, the use of optogenetics for longer periods, may not be the best approach, as the unavoidable surgical preparation with the chronic implantation of hardware for stimulation and prolonged exposure to highly energetic laser light will eventually culminate in confounding regional inflammation and tissue degradation. Therefore, we chose to use chemogenetics to examine the participation of sensory neurons in melanoma development, as it is more suitable to evaluate the long-term effects of sensory stimulations with less side-effects. Future studies may use similar approaches to explore the role of sensory neurons and other innervations in other cancers.

Our findings suggest that sensory neurons' overactivation affects the immune response to melanoma. Melanoma progression is influenced by the complex interplay between cancer cells and different components of the immune system [8]. Melanoma cells may cause disruption of the organism's immunity to overrun and escape the immune system control $[96,104]$. The role of sensory innervations in these interactions remains completely unknown. Lymphocytes are the dominant immune elements found infiltrating the melanoma microenvironment. Their composition correlates with patients' survival [38]. While regulatory $\mathrm{T}$ cells play pro-tumorigenic roles; $\mathrm{CD} 8+\mathrm{T}$ cells, $\mathrm{CD} 4+\mathrm{T}$ cells, $\gamma \delta \mathrm{T}$ cells, and NK cells have been shown to act against the transformed cells, [38, 43, 46, 47, 58, 64, 73, 118]. Conversely neutrophils and myeloid-derived suppressor cells have been associated with poor prognosis and are largely protumorigenic [22, 28, 69, 126, 129, 145, 155]. Our data shows that sensory overactivation induce an increase in the number of tumor-infiltrating anti-cancer lymphocytes $(\mathrm{CD} 8+\mathrm{T}$ cells, $\mathrm{CD} 4+\mathrm{T}$ cells, $\gamma \delta \mathrm{T}$ cells, and NK cells), while we did not detect changes in the number of tumor-infiltrating regulatory $\mathrm{T}$ cells. We also found that there is a decrease in the number of neutrophils and myeloid-derived suppressor cells within the tumor. We found that these changes were tumor-specific, as we did not detect any alterations in the number of lymphocytes in the non-draining lymph nodes. Tumor-draining lymph nodes presented an increase in some of the anti-tumor lymphocytes probably because of the tumor-priming effect previously reported [135]. Signals transmitted to T cells via PD-1 or CTLA-4 (considered markers for T cells "exhaustion") promote $\mathrm{T}$ cell dysfunction, thereby turning off the immune response $[59,98,149]$. We found that the tumor-infiltrating lymphocytes decrease their expression of PD1 and CTLA-4, possibly indicating that these cells are "less exhausted" within the melanoma microenvironment after sensory hyperactivation. The more active phenotypes of lymphocytes have been associated to the increase in the production of cytokines. A variety of lymphocytes are capable of producing IL-17 [14, 20, $24,53,56,77,94,103]$ which has presented anti-tumorigenic effects in melanoma $[3,63,74,75,91,99,100]$. We found an increase in tumor-infiltrating lymphocytes producing IL-17 after sensory stimulation. Thus, in light of our overall findings, we suggest that induced increase in firing of sensory innervations contributes to boosting 
A

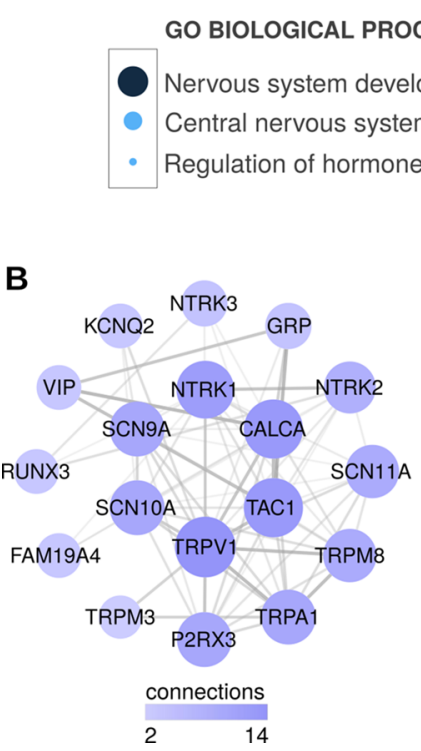

C

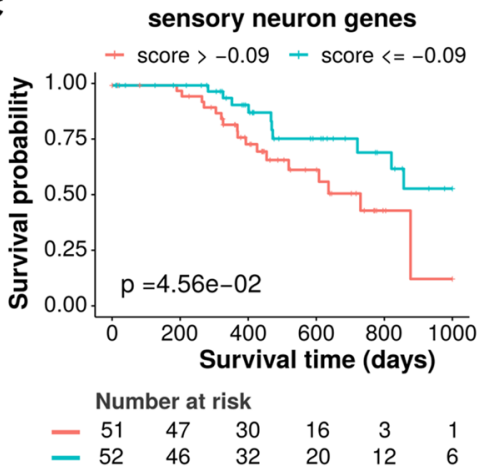

D

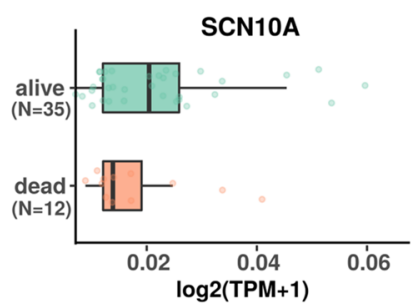

E

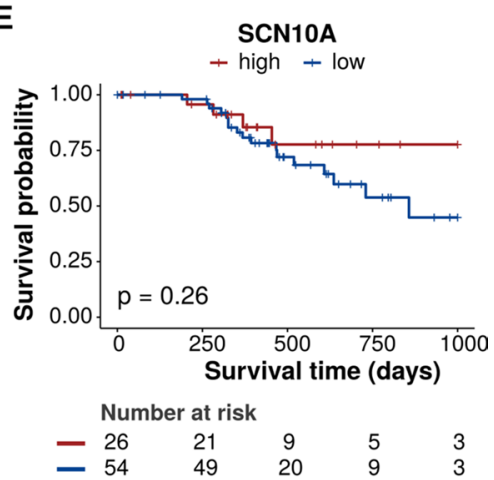

Fig. 8 Overexpression of genes related to sensory nerves is associated with Skin Cutaneous Melanomas (SKCM) patients improved survival. A Biological Processes of genes overexpressed in Skin Cutaneous Melanomas (SKCM) samples from alive patients versus dead patients. Patients were stratified (alive or dead) based on their vital status in a 5-year interval of their tumor diagnosis (clinical data available at TCGA and curated by Liu et al. (2018) [85]. We stratified patients in two groups, alive or dead, based on a 5-year interval of their tumor diagnosis. B Interactions among genes related to sensory neurons. C Gene signature using sensory neurons-related genes. High expression of these genes (lower patient scores) is associated with a better overall survival of SKCM patients (patients were stratified based on their median Reboot score). Overall survival of patients showing expression of sensory neurons-related genes. More negative scores are associated with higher gene expression. D Expression of SNC10A (Nav1.8) in SKCM samples. Only patients presenting tumors expressing SNC10A were used. E We evaluated the survival probability of patients with melanoma based on their tumor transcriptome. Patients were stratified based on lower/upper quartiles of SCN10A expression values. Overall survival of SKCM patients based on the level of expression of SCN10A. High expression of SCN10A (Nav1.8) correlates with best outcomes in patients with melanoma

of the immune response against melanoma. Future studies will need to explore the exact molecular mechanisms involved in the interactions of sensory neurons and immune cells in the melanoma microenvironment.

A variety of cellular and molecular mechanisms may be involved in the effect of sensory neurons' modulation on melanoma behavior. For instance, it has been documented that the same drug that is used to denervate sensory neurons, resiniferatoxin (RTX), an analogue of capsaicin, also induces stress by causing hyperactivation of the sympathetic nervous system $[16,62,157]$. These studies also revealed that sensory nerves may tune down sympathetic nerve activity [16, 62, 105]. Sympathetic neurons release norepinephrine [70, 123], which has been shown to strongly induce tumorigenesis $[1,60,154]$. It remains open the important question whether the effect of sensory innervations in the tumor microenvironment depend also on the modulation of the sympathetic tone.

\section{Future perspectives}

The present study reveals the short-term impact of chemogenetic modulation of sensory neurons on melanoma behavior. It remains to be examined what are the long-term effects of this manipulations. In the current study, the sensory neurons' activity is being continuously inhibited or overactivated. Are changes in sensory neurons' activity at specific time points sufficient to influence cancer outcomes? Also, it remains to be determined what are the changes within the tumor microenvironment at different stages of cancer progression. Are some stages more sensible to changes in the activity of sensory neurons? Moreover, this study focuses on melanoma tumors. Future studies should explore what is the role of sensory neurons in the development of other solid tumors.

A variety of factors secreted from sensory neurons may be implicated in the regulation of the melanoma microenvironment described here [18]. The overactivation of sensory nerve fibers may induce the release of neuropeptides, such as substance P, CGRP, VIP, GRP, neurokinin A, neurokinin $B$, neuropeptide $Y$ (NPY) and adrenomedullin, which have been shown to interact 
Table 3 Gene Ontology (GO) and analysis of Biological Processes (BP) in which specific genes are involved

\section{Up-regulated genes in alive $x$ dead}

Functional category

Nervous system development

Genes in list Total genes FDR

2474

1054

Central nervous system development

12

9

Regulation of hormone levels

Down-regulated genes in alive $x$ dead

Functional category

Cornification

Epithelium development

Genes in list Total genes

17

125

1386

Epithelial cell differentiation

33

831

1.67E-14

Tissue development

51

2168

2.40E-14

Skin development

23

464

18

268

Keratinization

Animal organ development

3779

410

521
Epidermis development

Keratinocyte differentiation

Ossification

Cellular developmental process

23

18

18

64

Cell differentiation
62

4459
Genes

0.004805005

0.029644095 CA10 KCNQ2 ACHE HPCAL4

0.029644095 ACHE PCSK1N MYB VGF RDH5 BCO1 TFAP2B KCNJ11 MYRIP

ARX HAPLN2 SLITRK5 VCX3A VCX ZNF365 NRTN TFAP2B NGFR MYB GFAP LRRTM2 LHFPL4 DPYSL5 FABP7 FOXG1 VIT MPZ

HAPLN2 VCX3A VCX ARX GFAP ZNF365 FABP7 SLITRK5 FOXG1 VIT CA10 HPCAL4 
Table 3 (continued)

\begin{tabular}{|c|c|c|c|c|}
\hline \multicolumn{5}{|l|}{ Down-regulated genes in alive $x$ dead } \\
\hline Functional category & Genes in list & Total genes & FDR & Genes \\
\hline Anatomical structure morphogenesis & 46 & 2785 & $1.00 \mathrm{E}-07$ & $\begin{array}{l}\text { PITX1 DLX5 KLK14 NGF HEYL RHOD NRARP HGF MYOC BMP4 IL1RN } \\
\text { FGF7 ZC3H12A GREM1 AJAP1 NOTCH3 WNT11 ANGPT2 SMPD3 ALOX12 } \\
\text { ZBTB16 MDFI SDC1 ID3 TBX4 KRT17 CHN1 CD36 GREB1L COL8A1 RSPO3 } \\
\text { FGF18 FBLIM1 PKDCC ELF3 TMEM79 ROR2 SIX2 SOX11 CCBE1 MAFB } \\
\text { KRT25 CREB3L1 JUP TBX1 ACTG2 }\end{array}$ \\
\hline Tissue morphogenesis & 21 & 719 & $8.87 \mathrm{E}-07$ & $\begin{array}{l}\text { KLK14 NRARP HGF BMP4 AJAP1 WNT11 ALOX12 TBX4 KRT17 FGF7 } \\
\text { GREB1L RSPO3 TMEM79 HEYL GREM1 ROR2 SIX2 SOX11 KRT25 ACTG2 } \\
\text { TBX1 }\end{array}$ \\
\hline Regulation of cartilage development & 8 & 68 & $1.39 \mathrm{E}-06$ & ZBTB16 BMP4 SMPD3 FGF18 PKDCC SIX2 GREM1 WNT11 \\
\hline Tube development & 25 & 1062 & $1.93 \mathrm{E}-06$ & $\begin{array}{l}\text { NRARP BMP4 FGF7 ZC3H12A HGF NOTCH3 WNT11 ANGPT2 SMPD3 } \\
\text { ALOX12 SDC1 TBX4 GREB1L COL8A1 RSPO3 FGF18 PKDCC HEYL GREM1 } \\
\text { SIX2 SOX11 CCBE1 CREB3L1 JUP TBX1 }\end{array}$ \\
\hline Morphogenesis of an epithelium & 18 & 581 & $3.91 \mathrm{E}-06$ & $\begin{array}{l}\text { KLK14 NRARP HGF BMP4 AJAP1 WNT11 ALOX12 TBX4 KRT17 FGF7 } \\
\text { GREB1L RSPO3 TMEM79 GREM1 ROR2 SIX2 SOX11 KRT25 }\end{array}$ \\
\hline Urogenital system development & 14 & 346 & 4.57E-06 & $\begin{array}{l}\text { SIX2 BMP4 GREB1L NOTCH3 WNT11 ANGPT2 ZBTB16 SDC1 ID3 } \\
\text { CYP19A1 HEYL GREM1 OVOL1 SOX11 }\end{array}$ \\
\hline Kidney development & 13 & 291 & 4.57E-06 & $\begin{array}{l}\text { SIX2 BMP4 GREB1L NOTCH3 WNT11 ANGPT2 ZBTB16 SDC1 ID3 HEYL } \\
\text { GREM1 OVOL1 SOX11 }\end{array}$ \\
\hline $\begin{array}{l}\text { Regulation of anatomical structure morpho- } \\
\text { genesis }\end{array}$ & 25 & 1125 & 4.57E-06 & $\begin{array}{l}\text { NGF RHOD HGF MYOC BMP4 IL1RN FGF7 ZC3H12A AJAP1 WNT11 } \\
\text { ANGPT2 ALOX12 CHN1 CD36 FBLIM1 GREM1 ROR2 SIX2 CCBE1 NRARP } \\
\text { FGF18 CREB3L1 JUP TBX1 RSPO3 }\end{array}$ \\
\hline Regulation of ossification & 11 & 193 & 4.57E-06 & $\begin{array}{l}\text { ZBTB16 BMP4 TWIST2 ID3 GDPD2 PKDCC ADRB2 SIX2 SOX11 GREM1 } \\
\text { HGF }\end{array}$ \\
\hline Skeletal system development & 17 & 541 & $6.10 \mathrm{E}-06$ & $\begin{array}{l}\text { ZBTB16 BMP4 MYOC PITX1 SMPD3 DLX5 MDFI FGF18 PKDCC ROR2 SIX2 } \\
\text { SOX11 PAPSS2 WNT11 TBX4 GREM1 TBX1 }\end{array}$ \\
\hline Renal system development & 13 & 307 & $7.35 E-06$ & $\begin{array}{l}\text { SIX2 BMP4 GREB1L NOTCH3 WNT11 ANGPT2 ZBTB16 SDC1 ID3 HEYL } \\
\text { GREM1 OVOL1 SOX11 }\end{array}$ \\
\hline Animal organ morphogenesis & 24 & 1099 & $1.06 \mathrm{E}-05$ & $\begin{array}{l}\text { HEYL HGF BMP4 FGF7 AJAP1 WNT11 SMPD3 DLX5 MDFI SDC1 ID3 } \\
\text { GREB1L COL8A1 FGF18 ELF3 GREM1 ROR2 SIX2 SOX11 MAFB TBX4 TBX1 } \\
\text { ACTG2 RSPO3 }\end{array}$ \\
\hline Osteoblast differentiation & 11 & 218 & $1.18 \mathrm{E}-05$ & $\begin{array}{l}\text { MYOC BMP4 TWIST2 WNT11 DLX5 ID3 GDPD2 GREM1 SOX11 CREB3L1 } \\
\text { HGF }\end{array}$ \\
\hline Regulation of developmental process & 41 & 2763 & $1.21 E-05$ & $\begin{array}{l}\text { ID3 NGF HEYL RHOD HGF MYOC ZBTB16 BMP4 IL1RN FGF7 ZC3H12A } \\
\text { AJAP1 MAFB TWIST2 NOTCH3 WNT11 ANGPT2 SMPD3 ALOX12 KRT17 } \\
\text { CHN1 GDPD2 CD36 FGF18 FBLIM1 PKDCC TMEM79 GREM1 ROR2 } \\
\text { ADRB2 SIX2 SOX11 CCBE1 NRARP CREB3L1 JUP SULT2B1 SIK1 S100A9 } \\
\text { TBX1 RSPO3 }\end{array}$ \\
\hline $\begin{array}{l}\text { Anatomical structure formation involved in } \\
\text { morphogenesis }\end{array}$ & 24 & 1164 & $2.67 E-05$ & $\begin{array}{l}\text { DLX5 NRARP BMP4 ZC3H12A HGF NOTCH3 WNT11 ANGPT2 SMPD3 } \\
\text { TBX4 CD36 COL8A1 RSPO3 FGF18 HEYL GREM1 ROR2 SIX2 SOX11 } \\
\text { CCBE1 MAFB CREB3L1 JUP TBX1 }\end{array}$ \\
\hline Skeletal system morphogenesis & 11 & 245 & $3.39 E-05$ & SMPD3 DLX5 MDFI BMP4 FGF18 ROR2 SIX2 SOX11 TBX4 GREM1 TBX1 \\
\hline Regulation of chondrocyte differentiation & 6 & 49 & $3.91 \mathrm{E}-05$ & BMP4 FGF18 PKDCC SIX2 ZBTB16 GREM1 \\
\hline Appendage morphogenesis & 9 & 155 & $3.91 \mathrm{E}-05$ & PITX1 DLX5 ZBTB16 TBX4 BMP4 PKDCC GREM1 ROR2 SOX11 \\
\hline Limb morphogenesis & 9 & 155 & $3.91 \mathrm{E}-05$ & PITX1 DLX5 ZBTB16 TBX4 BMP4 PKDCC GREM1 ROR2 SOX11 \\
\hline Tube morphogenesis & 20 & 860 & $3.91 \mathrm{E}-05$ & $\begin{array}{l}\text { NRARP BMP4 ZC3H12A HGF NOTCH3 WNT11 ANGPT2 ALOX12 TBX4 } \\
\text { GREB1L COL8A1 RSPO3 FGF18 GREM1 SIX2 SOX11 CCBE1 CREB3L1 JUP } \\
\text { TBX1 }\end{array}$ \\
\hline Regulation of morphogenesis of an epithelium & 10 & 204 & $4.32 \mathrm{E}-05$ & HGF BMP4 AJAP1 WNT11 ALOX12 FGF7 GREM1 ROR2 SIX2 RSPO3 \\
\hline Epidermis morphogenesis & 5 & 29 & $5.24 \mathrm{E}-05$ & KLK14 KRT17 FGF7 TMEM79 KRT25 \\
\hline Angiogenesis & 15 & 511 & $5.42 \mathrm{E}-05$ & $\begin{array}{l}\text { NRARP BMP4 ZC3H12A HGF NOTCH3 ANGPT2 TBX4 COL8A1 RSPO3 } \\
\text { FGF18 GREM1 CCBE1 CREB3L1 JUP TBX1 }\end{array}$ \\
\hline Cell migration & 27 & 1506 & $5.42 \mathrm{E}-05$ & $\begin{array}{l}\text { HGF SDC1 IL36G IL1RN DEFB4A RHOD MYOC ANGPT2 BMP4 FGF7 } \\
\text { FGF18 S100A9 ZC3H12A ROR2 SIX2 WNT11 BDKRB1 SMPD3 ALOX12 } \\
\text { CYP19A1 GREM1 CCBE1 LTB4R2 JUP TWIST2 TBX1 S100A12 }\end{array}$ \\
\hline Cell death & 36 & 2415 & $5.45 \mathrm{E}-05$ & $\begin{array}{l}\text { HGF BMP4 NGF ADAMTSL4 ZNF385B S100A9 ZC3H12A WNT11 ALOX12 } \\
\text { ZBTB16 ID3 CREB3L1 TMEM79 GREM1 SOX11 TWIST2 CD36 SIK1 TBX1 } \\
\text { PNMA5 TGM1 KRT37 PI3 KRT17 KLK14 TCHH KRT82 SPRR2D KLK13 } \\
\text { KRT80 JUP KLK12 KRT25 SPINK9 KRT81 SPRR2A }\end{array}$ \\
\hline Respiratory system development & 10 & 215 & $6.07 E-05$ & BMP4 FGF7 SMPD3 DLX5 TBX4 FGF18 PKDCC SOX11 CCBE1 WNT11 \\
\hline Cartilage development & 10 & 216 & $6.17 \mathrm{E}-05$ & ZBTB16 BMP4 PITX1 SMPD3 FGF18 PKDCC ROR2 SIX2 GREM1 WNT11 \\
\hline
\end{tabular}


Table 3 (continued)

\begin{tabular}{|c|c|c|c|c|}
\hline \multicolumn{5}{|l|}{ Down-regulated genes in alive $x$ dead } \\
\hline Functional category & Genes in list & Total genes & FDR & Genes \\
\hline Positive regulation of ossification & 7 & 88 & $6.78 \mathrm{E}-05$ & ZBTB16 BMP4 GDPD2 PKDCC ADRB2 SOX11 HGF \\
\hline $\begin{array}{l}\text { Regulation of multicellular organismal develop- } \\
\text { ment }\end{array}$ & 33 & 2138 & $7.25 \mathrm{E}-05$ & $\begin{array}{l}\text { ID3 NGF HEYL HGF ZBTB16 BMP4 IL1RN FGF7 ZC3H12A AJAP1 MAFB } \\
\text { NOTCH3 WNT11 ANGPT2 SMPD3 ALOX12 KRT17 CHN1 FGF18 PKDCC } \\
\text { GREM1 ROR2 ADRB2 SIX2 SOX11 CCBE1 NRARP CREB3L1 JUP SULT2B1 } \\
\text { S100A9 TBX1 RSPO3 }\end{array}$ \\
\hline Blood vessel morphogenesis & 16 & 603 & $7.46 \mathrm{E}-5$ & $\begin{array}{l}\text { NRARP BMP4 ZC3H12A HGF NOTCH3 WNT11 ANGPT2 TBX4 COL8A1 } \\
\text { RSPO3 FGF18 GREM1 CCBE1 CREB3L1 JUP TBX1 }\end{array}$ \\
\hline Programmed cell death & 34 & 2257 & $8.00 \mathrm{E}-05$ & $\begin{array}{l}\text { HGF BMP4 NGF ADAMTSL4 ZNF385B S100A9 WNT11 ALOX12 ZBTB16 } \\
\text { ID3 CREB3L1 TMEM79 ZC3H12A GREM1 TWIST2 SIK1 TBX1 PNMA5 } \\
\text { TGM1 KRT37 PI3 KRT17 KLK14 TCHH KRT82 SPRR2D KLK13 KRT80 JUP } \\
\text { KLK12 KRT25 SPINK9 KRT81 SPRR2A }\end{array}$ \\
\hline Blood vessel development & 17 & 687 & $8.58 \mathrm{E}-05$ & $\begin{array}{l}\text { HEYL NRARP BMP4 ZC3H12A HGF NOTCH3 WNT11 ANGPT2 TBX4 } \\
\text { COL8A1 RSPO3 FGF18 GREM1 CCBE1 CREB3L1 JUP TBX1 }\end{array}$ \\
\hline Embryonic morphogenesis & 16 & 615 & $8.98 \mathrm{E}-05$ & $\begin{array}{l}\text { BMP4 IL1RN PITX1 WNT11 DLX5 ZBTB16 MDFI TBX4 RSPO3 GREM1 } \\
\text { ROR2 SIX2 SOX11 MAFB COL8A1 TBX1 }\end{array}$ \\
\hline Cell motility & 28 & 1670 & $9.30 \mathrm{E}-05$ & $\begin{array}{l}\text { HGF SDC1 IL36G IL1RN DEFB4A RHOD MYOC ANGPT2 BMP4 FGF7 } \\
\text { FGF18 S100A9 ZC3H12A ROR2 SIX2 WNT11 BDKRB1 SMPD3 ALOX12 } \\
\text { CYP19A1 GREM1 CCBE1 LTB4R2 DUOX1 JUP TWIST2 TBX1 S100A12 }\end{array}$ \\
\hline Localization of cell & 28 & 1670 & $9.30 \mathrm{E}-05$ & $\begin{array}{l}\text { HGF SDC1 IL36G IL1RN DEFB4A RHOD MYOC ANGPT2 BMP4 FGF7 } \\
\text { FGF18 S100A9 ZC3H12A ROR2 SIX2 WNT11 BDKRB1 SMPD3 ALOX12 } \\
\text { CYP19A1 GREM1 CCBE1 LTB4R2 DUOX1 JUP TWIST2 TBX1 S100A12 }\end{array}$ \\
\hline Cellular response to growth factor stimulus & 17 & 694 & $9.30 \mathrm{E}-05$ & $\begin{array}{l}\text { HGF BMP4 NGF FGF18 CREB3L1 GREM1 CCBE1 FAM83G ANGPT2 SMPD3 } \\
\text { DLX5 RASL11B FGF7 HEYL ROR2 SOX11 TBX1 }\end{array}$ \\
\hline Cell-cell signaling & 29 & 1774 & $9.65 \mathrm{E}-05$ & $\begin{array}{l}\text { WNT11 CHRNA4 NGF RSPO3 NRARP JUP HGF CYP19A1 GREM1 FAM3D } \\
\text { SLC8A3 SMPD3 DLX5 SDC1 BMP4 IL1RN FGF7 ROR2 ADRB2 SOX11 } \\
\text { LYNX1 MYOC G6PC2 MDFI GLP1R IL36G FGF18 S100A9 ADRA1D }\end{array}$ \\
\hline Tissue migration & 11 & 293 & $1.10 E-04$ & $\begin{array}{l}\text { ANGPT2 BMP4 FGF7 FGF18 ZC3H12A ALOX12 GREM1 CCBE1 LTB4R2 } \\
\text { JUP ACTG2 }\end{array}$ \\
\hline Appendage development & 9 & 186 & 1.10E-04 & PITX1 DLX5 ZBTB16 TBX4 BMP4 PKDCC GREM1 ROR2 SOX11 \\
\hline Limb development & 9 & 186 & $1.10 \mathrm{E}-04$ & PITX1 DLX5 ZBTB16 TBX4 BMP4 PKDCC GREM1 ROR2 SOX11 \\
\hline Lung development & 9 & 188 & $1.18 \mathrm{E}-04$ & BMP4 FGF7 SMPD3 TBX4 FGF18 PKDCC SOX11 CCBE1 WNT11 \\
\hline Vasculature development & 17 & 715 & $1.20 \mathrm{E}-04$ & $\begin{array}{l}\text { HEYL NRARP BMP4 ZC3H12A HGF NOTCH3 WNT11 ANGPT2 TBX4 } \\
\text { COL8A1 RSPO3 FGF18 GREM1 CCBE1 CREB3L1 JUP TBX1 }\end{array}$ \\
\hline Respiratory tube development & 9 & 192 & $1.34 \mathrm{E}-04$ & BMP4 FGF7 SMPD3 TBX4 FGF18 PKDCC SOX11 CCBE1 WNT11 \\
\hline Response to growth factor & 17 & 723 & $1.34 \mathrm{E}-04$ & $\begin{array}{l}\text { HGF BMP4 NGF FGF18 CREB3L1 GREM1 CCBE1 FAM83G ANGPT2 SMPD3 } \\
\text { DLX5 RASL11B FGF7 HEYL ROR2 SOX11 TBX1 }\end{array}$ \\
\hline Cardiovascular system development & 17 & 724 & $1.34 \mathrm{E}-04$ & $\begin{array}{l}\text { HEYL NRARP BMP4 ZC3H12A HGF NOTCH3 WNT11 ANGPT2 TBX4 } \\
\text { COL8A1 RSPO3 FGF18 GREM1 CCBE1 CREB3L1 JUP TBX1 }\end{array}$ \\
\hline Locomotion & 30 & 1921 & $1.34 \mathrm{E}-04$ & $\begin{array}{l}\text { HGF SDC1 IL36G IL1RN DEFB4A RHOD MYOC ANGPT2 BMP4 FGF7 } \\
\text { FGF18 S100A9 ZC3H12A ROR2 SIX2 WNT11 BDKRB1 SMPD3 DLX5 } \\
\text { ALOX12 CHN1 CYP19A1 GREM1 CCBE1 LTB4R2 DUOX1 JUP TWIST2 } \\
\text { TBX1 S100A12 }\end{array}$ \\
\hline Mesonephros development & 7 & 104 & $1.40 \mathrm{E}-04$ & BMP4 ZBTB16 SDC1 GREB1L GREM1 SIX2 WNT11 \\
\hline Positive regulation of developmental process & 25 & 1433 & $1.40 \mathrm{E}-04$ & $\begin{array}{l}\text { NGF MYOC ZBTB16 BMP4 FGF7 ZC3H12A HGF ANGPT2 ALOX12 KRT17 } \\
\text { GDPD2 CD36 FGF18 PKDCC TMEM79 HEYL GREM1 ROR2 ADRB2 SOX11 } \\
\text { CCBE1 JUP SULT2B1 S100A9 TBX1 }\end{array}$ \\
\hline Nephron development & 8 & 147 & $1.41 \mathrm{E}-04$ & BMP4 NOTCH3 ANGPT2 GREB1L HEYL GREM1 SIX2 WNT11 \\
\hline Positive regulation of cell communication & 30 & 1937 & $1.48 \mathrm{E}-04$ & $\begin{array}{l}\text { BMP4 IL36G IL 1RN RSPO3 NRARP JUP HGF MYOC KLK14 CYP19A1 FGF18 } \\
\text { S100A9 S100A12 ZC3H12A ADRB2 ALOX12B CCBE1 SLC8A3 DLX5 CHN1 } \\
\text { CD36 GREM1 ROR2 SOX11 WNT11 SH3RF3 NGF TBX1 FGF7 ELF3 }\end{array}$ \\
\hline Positive regulation of signaling & 30 & 1945 & $1.56 \mathrm{E}-04$ & $\begin{array}{l}\text { BMP4 IL36G IL 1RN RSPO3 NRARP JUP HGF MYOC KLK14 CYP19A1 FGF18 } \\
\text { S100A9 S100A12 ZC3H12A ADRB2 ALOX12B CCBE1 SLC8A3 DLX5 CHN1 } \\
\text { CD36 GREM1 ROR2 SOX11 WNT11 SH3RF3 NGF TBX1 FGF7 ELF3 }\end{array}$ \\
\hline Circulatory system development & 21 & 1077 & $1.56 \mathrm{E}-04$ & $\begin{array}{l}\text { HEYL NRARP BMP4 ZC3H12A HGF NOTCH3 WNT11 ANGPT2 ID3 TBX4 } \\
\text { GREB1L COL8A1 RSPO3 FGF18 GREM1 SOX11 CCBE1 CREB3L1 JUP TBX1 } \\
\text { SIK1 }\end{array}$ \\
\hline Positive regulation of signal transduction & 28 & 1762 & $1.91 \mathrm{E}-04$ & $\begin{array}{l}\text { BMP4 IL36G IL1RN RSPO3 NRARP JUP HGF MYOC KLK14 FGF18 S100A9 } \\
\text { S100A12 ZC3H12A ADRB2 ALOX12B CCBE1 DLX5 CHN1 CD36 GREM1 } \\
\text { ROR2 SOX11 WNT11 SH3RF3 NGF TBX1 FGF7 ELF3 }\end{array}$ \\
\hline
\end{tabular}


Table 3 (continued)

\begin{tabular}{|c|c|c|c|c|}
\hline \multicolumn{5}{|l|}{ Down-regulated genes in alive $x$ dead } \\
\hline Functional category & Genes in list & Total genes & FDR & Genes \\
\hline Ameboidal-type cell migration & 12 & 392 & $2.39 \mathrm{E}-04$ & $\begin{array}{l}\text { ANGPT2 BMP4 FGF7 FGF18 ZC3H12A WNT11 ALOX12 GREM1 CCBE1 } \\
\text { LTB4R2 JUP TBX1 }\end{array}$ \\
\hline Regulation of osteoblast differentiation & 7 & 115 & $2.44 \mathrm{E}-04$ & BMP4 TWIST2 ID3 GDPD2 GREM1 SOX11 HGF \\
\hline Bone mineralization & 7 & 116 & $2.55 \mathrm{E}-04$ & BMP4 WNT11 SMPD3 PKDCC ROR2 ADRB2 GREM1 \\
\hline Establishment of skin barrier & 4 & 22 & $2.65 \mathrm{E}-04$ & ALOX12 TMEM79 ALOX12B CLDN4 \\
\hline Regulation of cell motility & 19 & 946 & $2.69 \mathrm{E}-04$ & $\begin{array}{l}\text { HGF RHOD MYOC ANGPT2 BMP4 FGF7 FGF18 ZC3H12A ROR2 BDKRB1 } \\
\text { SMPD3 ALOX12 CYP19A1 GREM1 CCBE1 DUOX1 JUP TWIST2 WNT11 }\end{array}$ \\
\hline Mesenchymal cell proliferation & 5 & 46 & $2.69 \mathrm{E}-04$ & BMP4 FGF7 WNT11 SIX2 TBX1 \\
\hline Positive regulation of cell motility & 14 & 544 & $2.73 \mathrm{E}-04$ & $\begin{array}{l}\text { HGF MYOC BMP4 FGF7 FGF18 ZC3H12A ROR2 BDKRB1 ALOX12 CCBE1 } \\
\text { DUOX1 TWIST2 WNT11 RHOD }\end{array}$ \\
\hline Response to BMP & 8 & 165 & $2.73 \mathrm{E}-04$ & BMP4 GREM1 FAM83G SMPD3 DLX5 HEYL ROR2 SOX11 \\
\hline Cellular response to BMP stimulus & 8 & 165 & $2.73 \mathrm{E}-04$ & BMP4 GREM1 FAM83G SMPD3 DLX5 HEYL ROR2 SOX11 \\
\hline Biomineral tissue development & 8 & 167 & 2.93E-04 & BMP4 WNT11 SMPD3 PKDCC ROR2 ADRB2 GREM1 TBX1 \\
\hline Connective tissue development & 10 & 280 & $3.18 \mathrm{E}-04$ & ZBTB16 BMP4 PITX1 SMPD3 FGF18 PKDCC ROR2 SIX2 GREM1 WNT11 \\
\hline Regulation of animal organ morphogenesis & 10 & 281 & $3.22 \mathrm{E}-04$ & HGF BMP4 FGF7 AJAP1 WNT11 GREM1 ROR2 SIX2 TBX1 RSPO3 \\
\hline Embryo development & 20 & 1054 & $3.22 \mathrm{E}-04$ & $\begin{array}{l}\text { BMP4 IL1RN PITX1 WNT11 DLX5 ZBTB16 MDFI ID3 TBX4 RSPO3 PKDCC } \\
\text { ELF3 GREM1 ROR2 SIX2 SOX11 NRARP MAFB COL8A1 TBX1 }\end{array}$ \\
\hline Chondrocyte differentiation & 7 & 123 & $3.22 \mathrm{E}-04$ & SMPD3 BMP4 FGF18 PKDCC SIX2 ZBTB16 GREM1 \\
\hline Epithelial cell migration & 10 & 284 & $3.33 \mathrm{E}-04$ & $\begin{array}{l}\text { ANGPT2 BMP4 FGF7 FGF18 ZC3H12A ALOX12 GREM1 CCBE1 LTB4R2 } \\
\text { JUP }\end{array}$ \\
\hline Regulation of cell migration & 18 & 883 & $3.33 \mathrm{E}-04$ & $\begin{array}{l}\text { HGF RHOD MYOC ANGPT2 BMP4 FGF7 FGF18 ZC3H12A ROR2 BDKRB1 } \\
\text { SMPD3 ALOX12 CYP19A1 GREM1 CCBE1 JUP TWIST2 WNT11 }\end{array}$ \\
\hline $\begin{array}{l}\text { Positive regulation of cellular component } \\
\text { movement }\end{array}$ & 14 & 558 & $3.33 \mathrm{E}-04$ & $\begin{array}{l}\text { HGF MYOC BMP4 FGF7 FGF18 ZC3H12A ROR2 BDKRB1 ALOX12 CCBE1 } \\
\text { DUOX1 TWIST2 WNT11 RHOD }\end{array}$ \\
\hline Epithelium migration & 10 & 287 & 3.60E-04 & $\begin{array}{l}\text { ANGPT2 BMP4 FGF7 FGF18 ZC3H12A ALOX12 GREM1 CCBE1 LTB4R2 } \\
\text { JUP }\end{array}$ \\
\hline Hair follicle morphogenesis & 4 & 25 & $3.68 \mathrm{E}-04$ & KRT17 FGF7 TMEM79 KRT25 \\
\hline Regulation of water loss via skin & 4 & 25 & $3.68 \mathrm{E}-04$ & ALOX12 TMEM79 ALOX12B CLDN4 \\
\hline Embryonic limb morphogenesis & 7 & 130 & $4.20 \mathrm{E}-04$ & PITX1 DLX5 ZBTB16 TBX4 BMP4 GREM1 ROR2 \\
\hline Embryonic appendage morphogenesis & 7 & 130 & $4.20 \mathrm{E}-04$ & PITX1 DLX5 ZBTB16 TBX4 BMP4 GREM1 ROR2 \\
\hline Positive regulation of locomotion & 14 & 576 & 4.34E-04 & $\begin{array}{l}\text { HGF MYOC BMP4 FGF7 FGF18 ZC3H12A ROR2 BDKRB1 ALOX12 CCBE1 } \\
\text { DUOX1 TWIST2 WNT11 RHOD }\end{array}$ \\
\hline Endochondral ossification & 4 & 27 & $4.78 \mathrm{E}-04$ & SMPD3 DLX5 BMP4 FGF18 \\
\hline Replacement ossification & 4 & 27 & 4.78E-04 & SMPD3 DLX5 BMP4 FGF18 \\
\hline Positive regulation of MAPK cascade & 14 & 583 & $4.79 E-04$ & $\begin{array}{l}\text { IL36G IL1RN BMP4 FGF18 ZC3H12A ADRB2 ALOX12B HGF CD36 ROR2 } \\
\text { SH3RF3 TBX1 NGF S100A12 }\end{array}$ \\
\hline Poly-N-acetyllactosamine biosynthetic process & 3 & 10 & $5.48 \mathrm{E}-04$ & B3GNT4 B3GNT8 B3GNT3 \\
\hline Regulation of multicellular organismal process & 41 & 3382 & $5.95 \mathrm{E}-04$ & $\begin{array}{l}\text { ID3 NGF IL36G IL1RN HEYL AVPR1A ADRB2 HGF ANGPT2 ZBTB16 BMP4 } \\
\text { FGF7 FGF18 ZC3H12A CCBE1 AJAP1 MAFB TWIST2 NOTCH3 WNT11 } \\
\text { SLC8A3 SMPD3 ALOX12 GLP1R KRT17 CHN1 GDPD2 CD36 PKDCC } \\
\text { GREM1 ROR2 SIX2 SOX11 NRARP CREB3L1 JUP SULT2B1 ALOX12B } \\
\text { S100A9 TBX1 RSPO3 }\end{array}$ \\
\hline Response to endogenous stimulus & 26 & 1704 & 5.97E-04 & $\begin{array}{l}\text { BMP4 NGF AVPR1A IL1RN FGF18 CREB3L1 HEYL GREM1 JUP FAM83G } \\
\text { SLC8A3 CHRNA4 SMPD3 DLX5 GLP1R SDC1 RASL11B CD36 FGF7 ROR2 } \\
\text { ADRB2 SOX11 CLDN4 TBX1 GNA15 CATSPERB }\end{array}$ \\
\hline Positive regulation of cell migration & 13 & 521 & $6.09 \mathrm{E}-04$ & $\begin{array}{l}\text { HGF MYOC BMP4 FGF7 FGF18 ZC3H12A ROR2 BDKRB1 ALOX12 CCBE1 } \\
\text { TWIST2 WNT11 RHOD }\end{array}$ \\
\hline Chemotaxis & 15 & 680 & $6.18 \mathrm{E}-04$ & $\begin{array}{l}\text { IL36G IL1RN DEFB4A HGF ANGPT2 FGF7 FGF18 S100A9 DLX5 BMP4 } \\
\text { CHN1 CYP19A1 GREM1 S100A12 LTB4R2 }\end{array}$ \\
\hline Regulation of cellular component movement & 19 & 1028 & $6.21 \mathrm{E}-04$ & $\begin{array}{l}\text { HGF RHOD MYOC ANGPT2 BMP4 FGF7 FGF18 ZC3H12A ROR2 BDKRB1 } \\
\text { SMPD3 ALOX12 CYP19A1 GREM1 CCBE1 DUOX1 JUP TWIST2 WNT11 }\end{array}$ \\
\hline Movement of cell or subcellular component & 30 & 2139 & $6.25 \mathrm{E}-04$ & $\begin{array}{l}\text { HGF SDC1 IL36G IL1RN DEFB4A RHOD MYOC ANGPT2 BMP4 FGF7 } \\
\text { FGF18 S100A9 ZC3H12A ROR2 SIX2 WNT11 BDKRB1 SMPD3 DLX5 } \\
\text { ALOX12 CHN1 CYP19A1 GREM1 CCBE1 LTB4R2 DUOX1 JUP TWIST2 } \\
\text { TBX1 S100A12 }\end{array}$ \\
\hline Taxis & 15 & 683 & $6.25 \mathrm{E}-04$ & $\begin{array}{l}\text { IL36G IL1RN DEFB4A HGF ANGPT2 FGF7 FGF18 S100A9 DLX5 BMP4 } \\
\text { CHN1 CYP19A1 GREM1 S100A12 LTB4R2 }\end{array}$ \\
\hline
\end{tabular}


Table 3 (continued)

\begin{tabular}{|c|c|c|c|c|}
\hline \multicolumn{5}{|l|}{ Down-regulated genes in alive $x$ dead } \\
\hline Functional category & Genes in list & Total genes & FDR & Genes \\
\hline Nephron tubule development & 6 & 96 & $6.25 \mathrm{E}-04$ & BMP4 GREB1L HEYL GREM1 SIX2 WNT11 \\
\hline Embryonic organ development & 12 & 452 & $6.34 \mathrm{E}-04$ & $\begin{array}{l}\text { WNT11 DLX5 MDFI ID3 BMP4 RSPO3 PKDCC ROR2 SIX2 SOX11 MAFB } \\
\text { TBX1 }\end{array}$ \\
\hline Morphogenesis of a branching epithelium & 8 & 194 & $6.34 \mathrm{E}-04$ & NRARP HGF BMP4 FGF7 GREB1L RSPO3 GREM1 SIX2 \\
\hline Kidney epithelium development & 7 & 143 & $6.55 \mathrm{E}-04$ & BMP4 SDC1 GREB1L HEYL GREM1 SIX2 WNT11 \\
\hline Poly-N-acetyllactosamine metabolic process & 3 & 11 & $6.64 \mathrm{E}-04$ & B3GNT4 B3GNT8 B3GNT3 \\
\hline $\begin{array}{l}\text { Positive regulation of intracellular signal } \\
\text { transduction }\end{array}$ & 20 & 1133 & $6.72 \mathrm{E}-04$ & $\begin{array}{l}\text { IL36G IL1RN HGF MYOC BMP4 FGF18 S100A9 S100A12 ZC3H12A ADRB2 } \\
\text { ALOX12B CD36 GREM1 ROR2 SOX11 WNT11 SH3RF3 NGF TBX1 FGF7 }\end{array}$ \\
\hline Regulation of locomotion & 19 & 1041 & $6.74 \mathrm{E}-04$ & $\begin{array}{l}\text { HGF RHOD MYOC ANGPT2 BMP4 FGF7 FGF18 ZC3H12A ROR2 BDKRB1 } \\
\text { SMPD3 ALOX12 CYP19A1 GREM1 CCBE1 DUOX1 JUP TWIST2 WNT11 }\end{array}$ \\
\hline Ureteric bud development & 6 & 99 & $6.85 \mathrm{E}-04$ & BMP4 SDC1 GREB1L GREM1 SIX2 WNT11 \\
\hline Renal tubule development & 6 & 99 & $6.85 \mathrm{E}-04$ & BMP4 GREB1L HEYL GREM1 SIX2 WNT11 \\
\hline Positive regulation of cartilage development & 4 & 31 & $6.94 \mathrm{E}-04$ & ZBTB16 BMP4 FGF18 PKDCC \\
\hline Mesonephric epithelium development & 6 & 100 & $7.06 \mathrm{E}-04$ & BMP4 SDC1 GREB1L GREM1 SIX2 WNT11 \\
\hline Mesonephric tubule development & 6 & 100 & 7.06E-04 & BMP4 SDC1 GREB1L GREM1 SIX2 WNT11 \\
\hline Positive regulation of gene expression & 29 & 2060 & $7.22 \mathrm{E}-04$ & $\begin{array}{l}\text { DLX5 ZBTB16 HEYL JUP SOX11 BMP4 ELF3 ZC3H12A CCBE1 PITX1 } \\
\text { NOTCH3 ALOX12 KRT17 CD36 FGF7 CREB3L1 GREM1 ROR2 ADRB2 SIX2 } \\
\text { OVOL1 MAFB WNT11 NGF ALOX12B GLIS3 ACTG2 TBX1 HGF }\end{array}$ \\
\hline $\begin{array}{l}\text { Regulation of morphogenesis of a branching } \\
\text { structure }\end{array}$ & 5 & 63 & $7.98 \mathrm{E}-04$ & HGF BMP4 FGF7 GREM1 SIX2 \\
\hline BMP signaling pathway & 7 & 152 & $8.66 \mathrm{E}-04$ & BMP4 GREM1 FAM83G SMPD3 DLX5 ROR2 SOX11 \\
\hline Endothelial cell migration & 8 & 206 & $8.66 \mathrm{E}-04$ & ANGPT2 BMP4 FGF18 ZC3H12A ALOX12 GREM1 CCBE1 JUP \\
\hline Morphogenesis of a branching structure & 8 & 208 & $9.13 \mathrm{E}-04$ & NRARP HGF BMP4 FGF7 GREB1L RSPO3 GREM1 SIX2 \\
\hline $\begin{array}{l}\text { Mesenchymal to epithelial transition involved in } \\
\text { metanephros morphogenesis }\end{array}$ & 3 & 13 & $1.01 E-03$ & BMP4 GREM1 SIX2 \\
\hline Mesenchymal cell differentiation & 8 & 212 & $1.01 \mathrm{E}-03$ & WNT11 BMP4 HEYL SIX2 SOX11 GREM1 TBX1 HGF \\
\hline Mesenchyme development & 9 & 273 & $1.01 E-03$ & WNT11 BMP4 HEYL SIX2 SOX11 GREM1 ACTG2 TBX1 HGF \\
\hline Ureteric bud morphogenesis & 5 & 67 & $1.01 E-03$ & BMP4 GREB1L GREM1 SIX2 WNT11 \\
\hline Mesonephric tubule morphogenesis & 5 & 68 & $1.08 \mathrm{E}-03$ & BMP4 GREB1L GREM1 SIX2 WNT11 \\
\hline Regulation of cell-substrate adhesion & 8 & 216 & $1.13 \mathrm{E}-03$ & MYOC CD36 AJAP1 ANGPT2 COL8A1 GREM1 RHOD JUP \\
\hline Regulation of endothelial cell migration & 7 & 161 & $1.16 \mathrm{E}-03$ & ANGPT2 BMP4 FGF18 ZC3H12A ALOX12 CCBE1 JUP \\
\hline $\begin{array}{l}\text { Positive regulation of multicellular organismal } \\
\text { process }\end{array}$ & 27 & 1911 & $1.17 \mathrm{E}-03$ & $\begin{array}{l}\text { NGF IL36G IL1RN AVPR1A ZBTB16 BMP4 FGF7 FGF18 ZC3H12A CCBE1 } \\
\text { HGF WNT11 ANGPT2 ALOX12 KRT17 GDPD2 CD36 PKDCC HEYL GREM1 } \\
\text { ROR2 ADRB2 SOX11 JUP ALOX12B S100A9 TBX1 }\end{array}$ \\
\hline Nephron epithelium development & 6 & 112 & $1.18 \mathrm{E}-03$ & BMP4 GREB1L HEYL GREM1 SIX2 WNT11 \\
\hline Regulation of epithelial cell migration & 8 & 223 & $1.36 \mathrm{E}-03$ & ANGPT2 BMP4 FGF7 FGF18 ZC3H12A ALOX12 CCBE1 JUP \\
\hline Positive regulation of epidermis development & 4 & 38 & $1.36 \mathrm{E}-03$ & BMP4 KRT17 TMEM79 SULT2B1 \\
\hline Aminoglycan biosynthetic process & 6 & 116 & $1.39 \mathrm{E}-03$ & B3GNT4 B3GNT8 B3GNT3 SMPD3 SDC1 HS3ST3A1 \\
\hline Positive regulation of angiogenesis & 7 & 167 & $1.39 \mathrm{E}-03$ & ZC3H12A HGF ANGPT2 GREM1 CCBE1 FGF18 JUP \\
\hline Sprouting angiogenesis & 6 & 117 & $1.45 \mathrm{E}-03$ & NRARP BMP4 RSPO3 GREM1 CREB3L1 CCBE1 \\
\hline Cell-substrate adhesion & 10 & 358 & $1.46 \mathrm{E}-03$ & LYPD3 LYPD5 MYOC CD36 AJAP1 ANGPT2 COL8A1 GREM1 RHOD JUP \\
\hline Sulfate assimilation & 2 & 3 & $1.52 \mathrm{E}-03$ & PAPSS2 SULT2B1 \\
\hline Cuticle development & 2 & 3 & $1.52 E-03$ & TMEM79 DUOX1 \\
\hline Cloacal septation & 2 & 3 & $1.52 \mathrm{E}-03$ & BMP4 WNT11 \\
\hline Aminoglycan metabolic process & 7 & 171 & $1.53 \mathrm{E}-03$ & B3GNT4 B3GNT8 B3GNT3 HGF SMPD3 SDC1 HS3ST3A1 \\
\hline Cardiac septum morphogenesis & 5 & 75 & $1.53 \mathrm{E}-03$ & HEYL WNT11 BMP4 SOX11 TBX1 \\
\hline Cellular response to endogenous stimulus & 22 & 1432 & $1.61 \mathrm{E}-03$ & $\begin{array}{l}\text { BMP4 NGF AVPR1A FGF18 CREB3L1 HEYL GREM1 JUP FAM83G SLC8A3 } \\
\text { CHRNA4 SMPD3 DLX5 GLP1R RASL11B FGF7 ROR2 ADRB2 SOX11 CD36 } \\
\text { TBX1 GNA15 }\end{array}$ \\
\hline Nephron tubule morphogenesis & 5 & 76 & $1.61 \mathrm{E}-03$ & BMP4 GREB1L GREM1 SIX2 WNT11 \\
\hline Metanephric renal vesicle morphogenesis & 3 & 16 & 1.65E-03 & BMP4 GREM1 SIX2 \\
\hline Regulation of cell-matrix adhesion & 6 & 122 & $1.68 \mathrm{E}-03$ & MYOC CD36 AJAP1 GREM1 RHOD JUP \\
\hline Outflow tract morphogenesis & 5 & 77 & $1.68 \mathrm{E}-03$ & WNT11 BMP4 HEYL SOX11 TBX1 \\
\hline Nephron epithelium morphogenesis & 5 & 78 & $1.77 \mathrm{E}-03$ & BMP4 GREB1L GREM1 SIX2 WNT11 \\
\hline
\end{tabular}


Table 3 (continued)

\section{Down-regulated genes in alive $x$ dead}

\begin{tabular}{llll}
\hline Functional category & Genes in list & Total genes & FDR \\
\hline Ventricular septum morphogenesis & 4 & 42 & $1.79 \mathrm{E}-03$ \\
Cell-matrix adhesion & 8 & 239 & $1.91 \mathrm{E}-03$ \\
Embryonic organ morphogenesis & 9 & 305 & $1.91 \mathrm{E}-03$ \\
Renal tubule morphogenesis & 5 & 80 & $1.91 \mathrm{E}-03$ \\
Nephron morphogenesis & 5 & 80 & $1.91 \mathrm{E}-03$ \\
Nephric duct development & 3 & 17 & $1.91 \mathrm{E}-03$ \\
Regulation of cell communication & 43 & 3903 & $2.15 \mathrm{E}-03$ \\
& & & \\
& & & \\
Enzyme linked receptor protein signaling & 18 & 1072 & $2.17 \mathrm{E}-03$ \\
pathway & & & \\
Regulation of MAPK cascade & 15 & 793 & $2.17 \mathrm{E}-03$ \\
Embryonic skeletal system development & 6 & 130 & $2.21 \mathrm{E}-03$ \\
Positive regulation of vasculature development & 7 & 186 & $2.29 \mathrm{E}-03$ \\
Response to organic substance & 40 & 3547 & $2.30 \mathrm{E}-03$ \\
& & &
\end{tabular}

\section{Positive chemotaxis}

Response to psychosocial stress

Cloaca development

Positive regulation of epithelial cell proliferation 7

Regulation of branching involved in salivary

gland morphogenesis by mesenchymal-epithe-

lial signaling

Myoblast differentiation

Regulation of signaling

5
2
2
7
2

43
84

4

4

190

87

3952

$2.62 \mathrm{E}-03$

$2.64 \mathrm{E}-03$

$2.56 \mathrm{E}-03$

\section{Genes}

HEYL WNT11 BMP4 SOX11

LYPD3 LYPD5 MYOC CD36 AJAP1 GREM1 RHOD JUP

WNT11 DLX5 MDFI BMP4 ROR2 SIX2 SOX11 MAFB TBX1

BMP4 GREB1L GREM1 SIX2 WNT11

BMP4 GREB1L GREM1 SIX2 WNT11

BMP4 GREB1L WNT11

BMP4 NGF IL36G IL1RN SIK1 RSPO3 NRARP JUP HGF MYOC KLK14 CYP19A1 FGF18 CREB3L1 S100A9 S100A12 ZC3H12A HEYL GREM1 ADRB2 ALOX12B LYNX1 CCBE1 FAM3D WNT11 SLC8A3 CHRNA4 DLX5 RASL11B CHN1 CD36 FGF7 AVPR1A ROR2 SOX11 G6PC2 SH3RF3 MDFI TBX1 NOTCH3 GLP1R ELF3 RHOD

HGF BMP4 NGF ROR2 ANGPT2 CREB3L1 GREM1 CCBE1 FAM83G MYOC SMPD3 DLX5 RASL11B FGF7 FGF18 SOX11 CHN1 ADRB2

BMP4 IL36G IL1RN MYOC FGF18 ZC3H12A ADRB2 ALOX12B HGF CD36 ROR2 SH3RF3 TBX1 NGF S100A12

MDFI BMP4 SIX2 SOX11 WNT11 TBX1

ZC3H12A HGF ANGPT2 GREM1 CCBE1 FGF18 JUP

HGF BMP4 NGF IL36G IL1RN AVPR1A CD36 DUOX1 FGF18 CREB3L1 ZC3H12A HEYL GREM1 JUP CCBE1 FAM83G ANGPT2 SLC8A3 BDKRB1 CHRNA4 SMPD3 DLX5 ALOX12 GLP1R SDC1 ID3 SLPI RASL11B FGF7 ROR2 ADRB2 ABCG4 SOX11 CLDN4 WNT11 TBX1 GNA15 CATSPERB HSPB3 GPX3

2.30E-03 ANGPT2 FGF7 HGF BMP4 DEFB4A

2.56E-03 GLP1RADRB2

BMP4 WNT11

NRARP BMP4 FGF7 DLX5 SOX11 TGM1 TBX1

HGF FGF7

BMP4 PITX1 SDC1 ID3 GREM 1

BMP4 NGF IL36G IL1RN SIK1 RSPO3 NRARP JUP HGF MYOC KLK14 CYP19A1 FGF18 CREB3L1 S100A9 S100A12 ZC3H12A HEYL GREM1 ADRB2 ALOX12B LYNX1 CCBE1 FAM3D WNT11 SLC8A3 CHRNA4 DLX5 RASL11B CHN1 CD36 FGF7 AVPR1A ROR2 SOX11 G6PC2 SH3RF3 MDFI TBX1 NOTCH3 GLP1R ELF3 RHOD

Negative regulation of transcription, DNA- $\quad 20$ templated

Positive regulation of chondrocyte differentiation

Mesenchymal to epithelial transition

Renal vesicle morphogenesis

Response to oxygen-containing compound

Regulation of vasculature development

Digestive tract morphogenesis

Metanephros development

Positive regulation of epithelial cell migration

Regulation of cell differentiation

Copulation

Positive regulation of cell differentiation

Renal vesicle development

in cell-cell signaling

$\begin{array}{ll}1298 & 2.73 \mathrm{E}-03 \\ 20 & 2.78 \mathrm{E}-03 \\ 20 & 2.78 \mathrm{E}-03 \\ 20 & 2.78 \mathrm{E}-03 \\ 1725 & 2.88 \mathrm{E}-03\end{array}$

$2.89 \mathrm{E}-03$

$3.01 \mathrm{E}-03$

$3.04 \mathrm{E}-03$

$3.04 \mathrm{E}-03$

3.04E-03

$3.06 \mathrm{E}-03$

$3.06 \mathrm{E}-03$

$3.06 \mathrm{E}-03$

$3.16 \mathrm{E}-03$
ZBTB16 BMP4 SIX2 OVOL1 SOX11 ZNF154 ID3 CREB3L1 ELF3 HEYL PITX1 NOTCH3 CD36 GREM1 NRARP USP2 WNT11 GLIS3 MDFI TWIST2 FGF18 PKDCC ZBTB16

BMP4 GREM1 SIX2

BMP4 GREM1 SIX2

IL36G IL1RN HGF DUOX1 ZC3H12A JUP ANGPT2 SLC8A3 BDKRB1 CHRNA4 SMPD3 GLP1R SDC1 ID3 SLPI CD36 AVPR1A ADRB2 CLDN4 WNT11 TBX1 GNA15 CATSPERB GPX3

BMP4 ZC3H12A HGF ANGPT2 GREM1 CCBE1 FGF18 CREB3L1 JUP BMP4 SIX2 SOX11 WNT11

BMP4 ID3 GREB1L GREM1 SIX2

BMP4 FGF7 FGF18 ZC3H12A ALOX12 CCBE1

ID3 NGF HEYL MYOC BMP4 ZC3H12A AJAP1 MAFB TWIST2 HGF NOTCH3 ZBTB16 CHN1 GDPD2 CD36 FGF18 PKDCC GREM1 ROR2 SIX2 SOX11 NRARP SULT2B1 SIK1 S100A9TBX1

KLK14 PI3 AVPR1A

NGF MYOC BMP4 ZC3H12A HGF ZBTB16 GDPD2 CD36 FGF18 PKDCC HEYL GREM1 ROR2 SOX11 SULT2B1 S100A9 TBX1

BMP4 GREM1 SIX2

WNT11 RSPO3 NRARP JUP GREM1 CHRNA4 DLX5 SDC1 ROR2 ADRB2 MYOC BMP4 MDFI 
Table 3 (continued)

\begin{tabular}{|c|c|c|c|c|}
\hline \multicolumn{5}{|l|}{ Down-regulated genes in alive $x$ dead } \\
\hline Functional category & Genes in list & Total genes & FDR & Genes \\
\hline Roof of mouth development & 5 & 93 & $3.25 \mathrm{E}-03$ & WNT11 DLX5 PKDCC SOX11 TBX1 \\
\hline Leukocyte migration & 11 & 491 & $3.36 \mathrm{E}-03$ & $\begin{array}{l}\text { IL36G IL1RN S100A9 BDKRB1 SMPD3 CYP19A1 GREM1 ROR2 ANGPT2 } \\
\text { SDC1 S100A12 }\end{array}$ \\
\hline Cell chemotaxis & 9 & 339 & $3.45 \mathrm{E}-03$ & IL36G IL1RN DEFB4A HGF FGF18 S100A9 CYP19A1 GREM1 S100A12 \\
\hline Sensory organ morphogenesis & 8 & 270 & $3.53 \mathrm{E}-03$ & DLX5 BMP4 COL8A1 ROR2 SIX2 SOX11 MAFB TBX1 \\
\hline $\begin{array}{l}\text { Negative regulation of cellular biosynthetic } \\
\text { process }\end{array}$ & 23 & 1658 & $3.67 \mathrm{E}-03$ & $\begin{array}{l}\text { ZBTB16 BMP4 SIX2 OVOL1 SOX11 ZNF154 ID3 CREB3L1 ELF3 ZC3H12A } \\
\text { HEYL PITX1 NOTCH3 SMPD3 CD36 GREM1 NRARP USP2 WNT11 GLIS3 } \\
\text { MDFI SIK1 TWIST2 }\end{array}$ \\
\hline Hepoxilin metabolic process & 2 & 5 & $3.67 \mathrm{E}-03$ & ALOX12 ALOX12B \\
\hline Hepoxilin biosynthetic process & 2 & 5 & $3.67 \mathrm{E}-03$ & ALOX12 ALOX12B \\
\hline Glomerulus vasculature morphogenesis & 2 & 5 & $3.67 \mathrm{E}-03$ & NOTCH3 BMP4 \\
\hline Glomerular capillary formation & 2 & 5 & $3.67 \mathrm{E}-03$ & NOTCH3 BMP4 \\
\hline Negative regulation of RNA metabolic process & 21 & 1446 & $3.68 \mathrm{E}-03$ & $\begin{array}{l}\text { ZBTB16 BMP4 SIX2 OVOL1 SOX11 ZNF154 ID3 CREB3L1 ELF3 ZC3H12A } \\
\text { HEYL PITX1 NOTCH3 CD36 GREM1 NRARP USP2 WNT11 GLIS3 MDFI } \\
\text { TWIST2 }\end{array}$ \\
\hline Embryonic skeletal system morphogenesis & 5 & 97 & $3.70 \mathrm{E}-03$ & MDFI BMP4 SIX2 SOX11 TBX1 \\
\hline Kidney morphogenesis & 5 & 97 & $3.70 E-03$ & BMP4 GREB1L GREM1 SIX2 WNT11 \\
\hline Cellular response to chemical stimulus & 39 & 3536 & $3.70 \mathrm{E}-03$ & $\begin{array}{l}\text { HGF BMP4 NGF IL36G IL1RN AVPR1A DEFB4A DUOX1 FGF18 CREB3L1 } \\
\text { S100A9 S100A12 ZC3H12A HEYL GREM1 JUP CCBE1 FAM83G ANGPT2 } \\
\text { SLC8A3 CHRNA4 SMPD3 DLX5 ALOX12 GLP1R ID3 RASL11B CD36 } \\
\text { CYP19A1 FGF7 ROR2 ADRB2 ABCG4 SOX11 GPX3 WNT11 TBX1 GNA15 } \\
\text { SDC1 }\end{array}$ \\
\hline Positive regulation of endothelial cell migration & 5 & 98 & $3.86 \mathrm{E}-03$ & BMP4 FGF18 ZC3H12A ALOX12 CCBE1 \\
\hline Regulation of cell adhesion & 14 & 765 & $3.94 \mathrm{E}-03$ & $\begin{array}{l}\text { MYOC CD36 IL1RN AJAP1 ANGPT2 ALOX12 ZBTB16 COL8A1 ZC3H12A } \\
\text { GREM1 RHOD NRARP JUP BMP4 }\end{array}$ \\
\hline $\begin{array}{l}\text { Negative regulation of nucleic acid-templated } \\
\text { transcription }\end{array}$ & 20 & 1353 & $3.96 \mathrm{E}-03$ & $\begin{array}{l}\text { ZBTB16 BMP4 SIX2 OVOL1 SOX11 ZNF154 ID3 CREB3L1 ELF3 HEYL } \\
\text { PITX1 NOTCH3 CD36 GREM1 NRARP USP2 WNT11 GLIS3 MDFITWIST2 }\end{array}$ \\
\hline Cellular response to organic substance & 34 & 2938 & $3.99 E-03$ & $\begin{array}{l}\text { HGF BMP4 NGF IL36G IL1RN AVPR1A DUOX1 FGF18 CREB3L1 ZC3H12A } \\
\text { HEYL GREM1 JUP CCBE1 FAM83G ANGPT2 SLC8A3 CHRNA4 SMPD3 } \\
\text { DLX5 ALOX12 GLP1R ID3 RASL11B CD36 FGF7 ROR2 ADRB2 ABCG4 } \\
\text { SOX11 WNT11 TBX1 GNA15 SDC1 }\end{array}$ \\
\hline Negative regulation of RNA biosynthetic process & 20 & 1355 & $3.99 E-03$ & $\begin{array}{l}\text { ZBTB16 BMP4 SIX2 OVOL1 SOX11 ZNF154 ID3 CREB3L1 ELF3 HEYL } \\
\text { PITX1 NOTCH3 CD36 GREM1 NRARP USP2 WNT11 GLIS3 MDFITWIST2 }\end{array}$ \\
\hline $\begin{array}{l}\text { Regulation of branching involved in ureteric } \\
\text { bud morphogenesis }\end{array}$ & 3 & 24 & $4.06 \mathrm{E}-03$ & BMP4 GREM1 SIX2 \\
\hline Intermediate filament organization & 3 & 24 & $4.06 \mathrm{E}-03$ & TCHH KRT17 KRT25 \\
\hline $\begin{array}{l}\text { Positive regulation of transcription by RNA } \\
\text { polymerase II }\end{array}$ & 19 & 1255 & 4.06E-03 & $\begin{array}{l}\text { DLX5 ZBTB16 HEYL JUP SOX11 BMP4 ZC3H12A PITX1 NOTCH3 CREB3L1 } \\
\text { ELF3 GREM1 ADRB2 SIX2 OVOL1 MAFB GLIS3 TBX1 HGF }\end{array}$ \\
\hline Glomerulus vasculature development & 3 & 24 & 4.06E-03 & BMP4 NOTCH3 ANGPT2 \\
\hline Negative regulation of biosynthetic process & 23 & 1681 & 4.10E-03 & $\begin{array}{l}\text { ZBTB16 BMP4 SIX2 OVOL1 SOX11 ZNF154 ID3 CREB3L1 ELF3 ZC3H12A } \\
\text { HEYL PITX1 NOTCH3 SMPD3 CD36 GREM1 NRARP USP2 WNT11 GLIS3 } \\
\text { MDFI SIK1 TWIST2 }\end{array}$ \\
\hline Carbohydrate biosynthetic process & 7 & 214 & 4.10E-03 & G6PC2 B3GNT4 B3GNT8 B3GNT3 SMPD3 CHST8 SIK1 \\
\hline Positive regulation of protein phosphorylation & 17 & 1057 & $4.10 \mathrm{E}-03$ & $\begin{array}{l}\text { HGF BMP4 IL36G IL1RN FGF7 FGF18 ZC3H12A ADRB2 ALOX12B WNT11 } \\
\text { CD36 GREM1 ROR2 SH3RF3 TBX1 NGF S100A12 }\end{array}$ \\
\hline $\begin{array}{l}\text { Negative regulation of nucleobasE-containing } \\
\text { compound metabolic process }\end{array}$ & 22 & 1575 & $4.15 \mathrm{E}-03$ & $\begin{array}{l}\text { ZBTB16 BMP4 SIX2 OVOL1 SOX11 ZNF154 ID3 CREB3L1 CDA ELF3 } \\
\text { ZC3H12A HEYL PITX1 NOTCH3 CD36 GREM1 NRARP USP2 WNT11 GLIS3 } \\
\text { MDFITWIST2 }\end{array}$ \\
\hline Regionalization & 9 & 355 & $4.24 \mathrm{E}-03$ & BMP4 ZBTB16 GREM1 ROR2 SIX2 NRARP MAFB MDFI TBX1 \\
\hline Regulation of protein import into nucleus & 4 & 58 & 4.41E-03 & BMP4 ZC3H12A JUP CD36 \\
\hline $\begin{array}{l}\text { Negative regulation of macromolecule biosyn- } \\
\text { thetic process }\end{array}$ & 22 & 1597 & $4.86 \mathrm{E}-03$ & $\begin{array}{l}\text { ZBTB16 BMP4 SIX2 OVOL1 SOX11 ZNF154 ID3 CREB3L1 ELF3 ZC3H12A } \\
\text { HEYL PITX1 NOTCH3 SMPD3 CD36 GREM1 NRARP USP2 WNT11 GLIS3 } \\
\text { MDFITWIST2 }\end{array}$ \\
\hline Positive regulation of osteoblast differentiation & 4 & 60 & 4.86E-03 & BMP4 GDPD2 SOX11 HGF \\
\hline $\begin{array}{l}\text { Positive regulation of transcription, DNA- } \\
\text { templated }\end{array}$ & 22 & 1599 & $4.86 \mathrm{E}-03$ & $\begin{array}{l}\text { DLX5 ZBTB16 HEYL JUP SOX11 BMP4 ELF3 ZC3H12A PITX1 NOTCH3 } \\
\text { FGF7 CREB3L1 GREM1 ROR2 ADRB2 SIX2 OVOL1 MAFB WNT11 GLIS3 } \\
\text { TBX1 HGF }\end{array}$ \\
\hline Renal system vasculature development & 3 & 26 & $4.86 \mathrm{E}-03$ & BMP4 NOTCH3 ANGPT2 \\
\hline
\end{tabular}


Table 3 (continued)

\begin{tabular}{|c|c|c|c|c|}
\hline \multicolumn{5}{|l|}{ Down-regulated genes in alive $x$ dead } \\
\hline Functional category & Genes in list & Total genes & FDR & Genes \\
\hline Kidney vasculature development & 3 & 26 & 4.86E-03 & BMP4 NOTCH3 ANGPT2 \\
\hline $\begin{array}{l}\text { Cell differentiation involved in metanephros } \\
\text { development }\end{array}$ & 3 & 26 & 4.86E -03 & BMP4 GREM1 SIX2 \\
\hline Metanephric nephron morphogenesis & 3 & 26 & $4.86 \mathrm{E}-03$ & BMP4 GREM1 SIX2 \\
\hline Glycosaminoglycan metabolic process & 6 & 161 & 4.99E-03 & HGF SMPD3 SDC1 HS3ST3A1 B3GNT4 B3GNT3 \\
\hline Regulation of protein import & 4 & 61 & 5.07E-03 & BMP4 ZC3H12A JUP CD36 \\
\hline $\begin{array}{l}\text { Branching involved in ureteric bud morpho- } \\
\text { genesis }\end{array}$ & 4 & 61 & $5.07 E-03$ & BMP4 GREB1L GREM1 SIX2 \\
\hline Glomerulus development & 4 & 61 & 5.07E-03 & BMP4 NOTCH3 ANGPT2 HEYL \\
\hline Negative regulation of cartilage development & 3 & 27 & $5.28 \mathrm{E}-03$ & BMP4 GREM1 WNT11 \\
\hline Regulation of mesonephros development & 3 & 27 & $5.28 \mathrm{E}-03$ & BMP4 GREM1 SIX2 \\
\hline Bone development & 7 & 228 & $5.49 \mathrm{E}-03$ & MYOC SMPD3 DLX5 BMP4 FGF18 PAPSS2 GREM1 \\
\hline $\begin{array}{l}\text { Negative regulation of cellular macromolecule } \\
\text { biosynthetic process }\end{array}$ & 21 & 1511 & $5.53 \mathrm{E}-03$ & $\begin{array}{l}\text { ZBTB16 BMP4 SIX2 OVOL1 SOX11 ZNF154 ID3 CREB3L1 ELF3 ZC3H12A } \\
\text { HEYL PITX1 NOTCH3 CD36 GREM1 NRARP USP2 WNT11 GLIS3 MDFI } \\
\text { TWIST2 }\end{array}$ \\
\hline Regulation of angiogenesis & 8 & 298 & $5.53 \mathrm{E}-03$ & ZC3H12A HGF ANGPT2 GREM1 CCBE1 FGF18 CREB3L1 JUP \\
\hline Peptide cross-linking & 4 & 63 & 5.57E-03 & SPRR2D SPRR2A TGM1 PI3 \\
\hline Embryonic hindlimb morphogenesis & 3 & 28 & $5.73 \mathrm{E}-03$ & PITX1 ZBTB16 BMP4 \\
\hline Secondary palate development & 3 & 28 & $5.73 \mathrm{E}-03$ & SOX11 WNT11 TBX1 \\
\hline Glycosaminoglycan biosynthetic process & 5 & 111 & $5.78 \mathrm{E}-03$ & SMPD3 SDC1 HS3ST3A1 B3GNT4 B3GNT3 \\
\hline Artery morphogenesis & 4 & 64 & $5.81 \mathrm{E}-03$ & BMP4 NOTCH3 WNT11 TBX1 \\
\hline Regulation of localization & 33 & 2905 & $5.84 \mathrm{E}-03$ & $\begin{array}{l}\text { HGF RHOD MYOC ANGPT2 BMP4 CYP19A1 FGF7 FGF18 ZC3H12A ROR2 } \\
\text { JUP FAM3D BDKRB1 CHRNA4 SMPD3 ALOX12 CD36 PKDCC AVPR1A } \\
\text { GREM1 ADRB2 CLIC3 SOX11 CCBE1 KCNK12 DUOX1 TWIST2 WNT11 } \\
\text { SDC1 G6PC2 ALOX12B SIK1 GLP1R }\end{array}$ \\
\hline Cardiac septum development & 5 & 112 & 5.93E-03 & HEYL WNT11 BMP4 SOX11 TBX1 \\
\hline $\begin{array}{l}\text { Positive regulation of cellular biosynthetic } \\
\text { process }\end{array}$ & 26 & 2082 & 5.95E-03 & $\begin{array}{l}\text { DLX5 ZBTB16 HEYL JUP SOX11 HGF BMP4 ELF3 ZC3H12A GREM1 PITX1 } \\
\text { NOTCH3 KRT17 CD36 FGF7 CREB3L1 AVPR1A ROR2 ADRB2 SIX2 OVOL1 } \\
\text { MAFB WNT11 GLIS3 TBX1 SMPD3 }\end{array}$ \\
\hline Response to lipid & 16 & 1006 & 5.95E-03 & $\begin{array}{l}\text { IL36G IL1RN ZC3H12A HEYL BDKRB1 ALOX12 SDC1 ID3 SLPI CD36 } \\
\text { AVPR1A CLDN4 BMP4 WNT11 TBX1 CATSPERB }\end{array}$ \\
\hline MAPK cascade & 16 & 1007 & 5.97E-03 & $\begin{array}{l}\text { BMP4 IL36G IL1RN MYOC FGF18 ZC3H12A ADRB2 ALOX12B HGF CD36 } \\
\text { ROR2 SH3RF3 TBX1 NGF FGF7 S100A12 }\end{array}$ \\
\hline Negative regulation of cell adhesion & 8 & 304 & $5.99 \mathrm{E}-03$ & MYOC IL1RN AJAP1 ANGPT2 ALOX12 ZC3H12A NRARP BMP4 \\
\hline Renal system vasculature morphogenesis & 2 & 7 & $6.07 \mathrm{E}-03$ & NOTCH3 BMP4 \\
\hline Kidney vasculature morphogenesis & 2 & 7 & 6.07E-03 & NOTCH3 BMP4 \\
\hline Positive regulation of phosphorylation & 17 & 1111 & $6.10 E-03$ & $\begin{array}{l}\text { HGF BMP4 IL36G IL1RN FGF7 FGF18 ZC3H12A ADRB2 ALOX12B WNT11 } \\
\text { CD36 GREM1 ROR2 SH3RF3 TBX1 NGF S100A12 }\end{array}$ \\
\hline Regulation of cell proliferation & 23 & 1756 & $6.32 \mathrm{E}-03$ & $\begin{array}{l}\text { NRARP BMP4 FGF7 GREM1 JUP NOTCH3 WNT11 SMPD3 DLX5 ALOX12 } \\
\text { ZBTB16 RARRES1 FGF18 AVPR1A SIX2 OVOL1 SOX11 SULT2B1 TGM1 } \\
\text { ROR2 TBX1 NGF ADRA1D }\end{array}$ \\
\hline $\begin{array}{l}\text { Negative regulation of nitrogen compound } \\
\text { metabolic process }\end{array}$ & 30 & 2564 & $6.32 \mathrm{E}-03$ & $\begin{array}{l}\text { ZBTB16 BMP4 SIX2 OVOL1 SOX11 ZNF154 HGF ID3 CREB3L1 CDA ELF3 } \\
\text { ZC3H12A HEYL GREM1 PITX1 NOTCH3 BDKRB1 SMPD3 PI3 SLPI NGF } \\
\text { CD36 NRARP SPINK9 COL28A1 USP2 WNT11 GLIS3 MDFI TWIST2 }\end{array}$ \\
\hline Cardiac chamber development & 6 & 172 & $6.32 \mathrm{E}-03$ & HEYL WNT11 BMP4 GREB1L SOX11 TBX1 \\
\hline Negative regulation of developmental process & 16 & 1017 & $6.40 E-03$ & $\begin{array}{l}\text { ID3 BMP4 MAFB TWIST2 NOTCH3 ANGPT2 ZBTB16 ZC3H12A ADRB2 } \\
\text { SIX2 SOX11 NRARP CREB3L1 GREM1 TBX1 WNT11 }\end{array}$ \\
\hline Signal transduction by protein phosphorylation & 16 & 1018 & $6.44 \mathrm{E}-03$ & $\begin{array}{l}\text { BMP4 IL36G IL1RN MYOC FGF18 ZC3H12A ADRB2 ALOX12B HGF CD36 } \\
\text { ROR2 SH3RF3 TBX1 NGF FGF7 S100A12 }\end{array}$ \\
\hline $\begin{array}{l}\text { Transmembrane receptor protein tyrosine } \\
\text { kinase signaling pathway }\end{array}$ & 13 & 730 & $6.45 \mathrm{E}-03$ & $\begin{array}{l}\text { HGF NGF ROR2 ANGPT2 CREB3L1 CCBE1 MYOC SMPD3 FGF7 FGF18 } \\
\text { GREM1 CHN1 ADRB2 }\end{array}$ \\
\hline Cell adhesion & 21 & 1541 & $6.46 \mathrm{E}-03$ & $\begin{array}{l}\text { LYPD3 LYPD5 MYOC CD36 IL1RN S100A9 JUP AJAP1 ANGPT2 ALOX12 } \\
\text { ZBTB16 COL8A1 FBLIM1 ZC3H12A GREM1 RHOD NRARP COL28A1 } \\
\text { BMP4 CLDN4 LRRN2 }\end{array}$ \\
\hline Epithelial cell proliferation & 9 & 387 & $6.56 \mathrm{E}-03$ & NRARP BMP4 FGF7 HGF DLX5 COL8A1 SOX11 TGM1 TBX1 \\
\hline
\end{tabular}


Table 3 (continued)

\begin{tabular}{|c|c|c|c|c|}
\hline \multicolumn{5}{|l|}{ Down-regulated genes in alive $x$ dead } \\
\hline Functional category & Genes in list & Total genes & FDR & Genes \\
\hline Biological adhesion & 21 & 1548 & $6.78 \mathrm{E}-03$ & $\begin{array}{l}\text { LYPD3 LYPD5 MYOC CD36 IL1RN S100A9 JUP AJAP1 ANGPT2 ALOX12 } \\
\text { ZBTB16 COL8A1 FBLIM1 ZC3H12A GREM1 RHOD NRARP COL28A1 } \\
\text { BMP4 CLDN4 LRRN2 }\end{array}$ \\
\hline Myeloid leukocyte migration & 7 & 241 & $6.79 E-03$ & IL36G IL1RN S100A9 CYP19A1 GREM1 ROR2 S100A12 \\
\hline Positive regulation of biosynthetic process & 26 & 2113 & $6.91 \mathrm{E}-03$ & $\begin{array}{l}\text { DLX5 ZBTB16 HEYL JUP SOX11 HGF BMP4 CREB3L1 ELF3 ZC3H12A } \\
\text { GREM1 PITX1 NOTCH3 KRT17 CD36 FGF7 AVPR1A ROR2 ADRB2 SIX2 } \\
\text { OVOL1 MAFB WNT11 GLIS3 TBX1 SMPD3 }\end{array}$ \\
\hline Blood vessel endothelial cell migration & 5 & 119 & $7.19 E-03$ & ANGPT2 ALOX12 GREM1 FGF18 JUP \\
\hline $\begin{array}{l}\text { Mesenchymal cell differentiation involved in } \\
\text { renal system development }\end{array}$ & 2 & 8 & $7.48 \mathrm{E}-03$ & BMP4 SIX2 \\
\hline Mesenchymal-epithelial cell signaling & 2 & 8 & $7.48 \mathrm{E}-03$ & HGF FGF7 \\
\hline Glomerulus morphogenesis & 2 & 8 & $7.48 \mathrm{E}-03$ & NOTCH3 BMP4 \\
\hline $\begin{array}{l}\text { Mesenchymal cell differentiation involved in } \\
\text { kidney development }\end{array}$ & 2 & 8 & $7.48 \mathrm{E}-03$ & BMP4 SIX2 \\
\hline $\begin{array}{l}\text { Negative regulation of mesenchymal cell } \\
\text { proliferation }\end{array}$ & 2 & 8 & $7.48 \mathrm{E}-03$ & BMP4 WNT11 \\
\hline $\begin{array}{l}\text { Negative regulation of cellular metabolic } \\
\text { process }\end{array}$ & 31 & 2724 & $7.59 \mathrm{E}-03$ & $\begin{array}{l}\text { ZBTB16 BMP4 SIX2 OVOL1 SOX11 ZNF154 HGF ID3 CREB3L1 CDA ELF3 } \\
\text { ZC3H12A HEYL GREM1 PITX1 NOTCH3 BDKRB1 SMPD3 PI3 SLPI NGF } \\
\text { CD36 NRARP SPINK9 COL28A1 USP2 WNT11 GLIS3 MDFI SIK1 TWIST2 }\end{array}$ \\
\hline Multicellular organismal water homeostasis & 4 & 71 & 7.63E-03 & ALOX12TMEM79 ALOX12B CLDN4 \\
\hline Endochondral bone morphogenesis & 4 & 72 & $8.01 \mathrm{E}-03$ & SMPD3 DLX5 BMP4 FGF18 \\
\hline Metanephros morphogenesis & 3 & 33 & $8.15 E-03$ & BMP4 GREM1 SIX2 \\
\hline $\begin{array}{l}\text { Positive regulation of nucleic acid-templated } \\
\text { transcription }\end{array}$ & 22 & 1694 & $8.53 \mathrm{E}-03$ & $\begin{array}{l}\text { DLX5 ZBTB16 HEYL JUP SOX11 BMP4 ELF3 ZC3H12A PITX1 NOTCH3 } \\
\text { FGF7 CREB3L1 GREM1 ROR2 ADRB2 SIX2 OVOL1 MAFB WNT11 GLIS3 } \\
\text { TBX1 HGF }\end{array}$ \\
\hline Positive regulation of RNA biosynthetic process & 22 & 1695 & $8.56 \mathrm{E}-03$ & $\begin{array}{l}\text { DLX5 ZBTB16 HEYL JUP SOX11 BMP4 ELF3 ZC3H12A PITX1 NOTCH3 } \\
\text { FGF7 CREB3L1 GREM1 ROR2 ADRB2 SIX2 OVOL1 MAFB WNT11 GLIS3 } \\
\text { TBX1 HGF }\end{array}$ \\
\hline $\begin{array}{l}\text { Positive regulation of morphogenesis of an } \\
\text { epithelium }\end{array}$ & 3 & 34 & $8.72 \mathrm{E}-03$ & ALOX12 BMP4 GREM1 \\
\hline Regulation of mesenchymal cell proliferation & 3 & 34 & $8.72 \mathrm{E}-03$ & BMP4 WNT11 TBX1 \\
\hline Ventricular septum development & 4 & 74 & $8.72 \mathrm{E}-03$ & HEYL WNT11 BMP4 SOX11 \\
\hline Ear morphogenesis & 5 & 127 & $9.06 \mathrm{E}-03$ & DLX5 ROR2 SIX2 MAFB TBX1 \\
\hline Cell proliferation & 26 & 2165 & $9.07 \mathrm{E}-03$ & $\begin{array}{l}\text { NRARP DLX5 BMP4 FGF7 GREM1 SIX2 JUP HGF NOTCH3 WNT11 SMPD3 } \\
\text { ALOX12 ZBTB16 RARRES1 COL8A1 FGF18 AVPR1A OVOL1 SOX11 } \\
\text { SULT2B1 TGM1 SDCBP2 ROR2 TBX1 NGF ADRA1D }\end{array}$ \\
\hline Soft palate development & 2 & 9 & $9.07 \mathrm{E}-03$ & SOX11 TBX1 \\
\hline $\begin{array}{l}\text { Regulation of branching involved in salivary } \\
\text { gland morphogenesis }\end{array}$ & 2 & 9 & $9.07 \mathrm{E}-03$ & HGF FGF7 \\
\hline Mesonephric duct development & 2 & 9 & 9.07E-03 & GREB1L WNT11 \\
\hline $\begin{array}{l}\text { Regulation of DNA-binding transcription factor } \\
\text { activity }\end{array}$ & 10 & 495 & $9.09 \mathrm{E}-03$ & ID3 ZC3H12A HEYL JUP GREM1 CD36 SIK1 MDFI S100A9 S100A12 \\
\hline Regulation of hormone levels & 11 & 583 & $9.12 \mathrm{E}-03$ & $\begin{array}{l}\text { CYP19A1 FAM3D SMPD3 IL1RN DUOX1 CRABP1 SOX11 CHST8 G6PC2 } \\
\text { GLP1R NGF }\end{array}$ \\
\hline Water homeostasis & 4 & 76 & $9.28 \mathrm{E}-03$ & ALOX12 TMEM79 ALOX12B CLDN4 \\
\hline Cellular response to toxic substance & 7 & 259 & $9.32 \mathrm{E}-03$ & HGF SMPD3 CD36 DUOX1 S100A9 ZC3H12A GPX3 \\
\hline Cardiac chamber morphogenesis & 5 & 129 & $9.38 \mathrm{E}-03$ & HEYL WNT11 BMP4 SOX11 TBX1 \\
\hline Cardiac ventricle development & 5 & 130 & 9.67E-03 & HEYL WNT11 BMP4 GREB1L SOX11 \\
\hline Regulation of signal transduction & 37 & 3529 & $9.74 \mathrm{E}-03$ & $\begin{array}{l}\text { BMP4 IL36G IL1RN SIK1 RSPO3 NRARP JUP HGF MYOC KLK14 FGF18 } \\
\text { CREB3L1 S100A9 S100A12 ZC3H12A HEYL GREM1 ADRB2 ALOX12B } \\
\text { LYNX1 CCBE1 WNT11 DLX5 RASL11B CHN1 NGF CD36 FGF7 ROR2 } \\
\text { SOX11 FAM3D SH3RF3 MDFI TBX1 NOTCH3 ELF3 RHOD }\end{array}$ \\
\hline Hindlimb morphogenesis & 3 & 36 & $9.82 \mathrm{E}-03$ & PITX1 ZBTB16 BMP4 \\
\hline Odontogenesis & 5 & 131 & $9.89 \mathrm{E}-03$ & SMPD3 SDC1 ID3 BMP4 TBX1 \\
\hline Regulation of bone mineralization & 4 & 78 & $9.90 \mathrm{E}-03$ & BMP4 PKDCC ADRB2 GREM1 \\
\hline Polysaccharide biosynthetic process & 4 & 78 & $9.90 \mathrm{E}-03$ & B3GNT4 B3GNT8 B3GNT3 SMPD3 \\
\hline
\end{tabular}


Table 3 (continued)

\begin{tabular}{|c|c|c|c|c|}
\hline \multicolumn{5}{|l|}{ Down-regulated genes in alive $x$ dead } \\
\hline Functional category & Genes in list & Total genes & FDR & Genes \\
\hline $\begin{array}{l}\text { Negative regulation of multicellular organismal } \\
\text { process }\end{array}$ & 18 & 1286 & $9.90 \mathrm{E}-03$ & $\begin{array}{l}\text { ID3 ADRB2 ANGPT2 BMP4 ZC3H12A MAFB TWIST2 HGF NOTCH3 WNT11 } \\
\text { ALOX12 ZBTB16 AVPR1A SOX11 NRARP CREB3L1 JUP GREM1 }\end{array}$ \\
\hline Regulation of protein phosphorylation & 20 & 1503 & $9.92 \mathrm{E}-03$ & $\begin{array}{l}\text { HGF BMP4 IL36G IL1RN MYOC FGF7 FGF18 ZC3H12A GREM1 ADRB2 } \\
\text { ALOX12B WNT11 BDKRB1 SMPD3 CD36 ROR2 SH3RF3 TBX1 NGF } \\
\text { S100A12 }\end{array}$ \\
\hline Cell surface receptor signaling pathway & 35 & 3287 & $1.00 \mathrm{E}-02$ & $\begin{array}{l}\text { HGF WNT11 BMP4 NGF IL36G IL1RN RSPO3 HEYL ROR2 NRARP JUP } \\
\text { ANGPT2 DUOX1 CREB3L1 GREM1 CCBE1 FAM83G MYOC NOTCH3 } \\
\text { CHRNA4 SMPD3 DLX5 GLP1R SDC1 RASL11B CD36 FGF7 FGF18 } \\
\text { ZC3H12A ADRB2 SOX11 MDFI CHN1 CDA ELF3 }\end{array}$ \\
\hline $\begin{array}{l}\text { Positive regulation of phosphorus metabolic } \\
\text { process }\end{array}$ & 17 & 1183 & $1.00 \mathrm{E}-02$ & $\begin{array}{l}\text { HGF BMP4 IL36G IL1RN FGF7 FGF18 ZC3H12A ADRB2 ALOX12B WNT11 } \\
\text { CD36 GREM1 ROR2 SH3RF3 TBX1 NGF S100A12 }\end{array}$ \\
\hline $\begin{array}{l}\text { Positive regulation of phosphate metabolic } \\
\text { process }\end{array}$ & 17 & 1183 & $1.00 \mathrm{E}-02$ & $\begin{array}{l}\text { HGF BMP4 IL36G IL1RN FGF7 FGF18 ZC3H12A ADRB2 ALOX12B WNT11 } \\
\text { CD36 GREM1 ROR2 SH3RF3 TBX1 NGF S100A12 }\end{array}$ \\
\hline $\begin{array}{l}\text { Cellular response to oxidised low-density lipo- } \\
\text { protein particle stimulus }\end{array}$ & 2 & 10 & $1.05 \mathrm{E}-02$ & SMPD3 CD36 \\
\hline Positive regulation of keratinocyte proliferation & 2 & 10 & $1.05 \mathrm{E}-02$ & FGF7TGM1 \\
\hline $\begin{array}{l}\text { Positive regulation of mitochondrial depolariza- } \\
\text { tion }\end{array}$ & 2 & 10 & $1.05 E-02$ & MYOC ALOX12 \\
\hline $\begin{array}{l}\text { Epithelial cell proliferation involved in lung } \\
\text { morphogenesis }\end{array}$ & 2 & 10 & $1.05 \mathrm{E}-02$ & BMP4 FGF7 \\
\hline Epithelial tube morphogenesis & 8 & 344 & $1.06 \mathrm{E}-02$ & NRARP BMP4 ALOX12 GREB1L GREM1 SIX2 SOX11 WNT11 \\
\hline Epithelial to mesenchymal transition & 5 & 135 & $1.08 \mathrm{E}-02$ & WNT11 BMP4 HEYL GREM1 HGF \\
\hline Negative regulation of growth & 7 & 269 & $1.08 \mathrm{E}-02$ & BMP4 CDA BDKRB1 CD36 GREM1 ADRB2 WNT11 \\
\hline Mesoderm development & 5 & 136 & $1.11 \mathrm{E}-02$ & BMP4 WNT11 SIX2 OVOL1 TBX1 \\
\hline Canonical Wnt signaling pathway & 8 & 350 & $1.16 \mathrm{E}-02$ & RSPO3 NRARP JUP GREM1 WNT11 DLX5 SDC1 ROR2 \\
\hline Digestive tract development & 5 & 139 & $1.21 \mathrm{E}-02$ & BMP4 PKDCC SIX2 SOX11 WNT11 \\
\hline Mesenchymal cell development & 4 & 84 & $1.23 \mathrm{E}-02$ & BMP4 HEYL SOX11 TBX1 \\
\hline Positive regulation of bone mineralization & 3 & 40 & $1.24 \mathrm{E}-02$ & BMP4 PKDCC ADRB2 \\
\hline Embryonic digestive tract development & 3 & 40 & $1.24 \mathrm{E}-02$ & PKDCC SIX2 SOX11 \\
\hline $\begin{array}{l}\text { Regulation of cellular response to growth factor } \\
\text { stimulus }\end{array}$ & 7 & 277 & $1.24 \mathrm{E}-02$ & FGF18 CREB3L1 GREM1 CCBE1 BMP4 RASL11B SOX11 \\
\hline Cell fate commitment & 7 & 277 & 1.24E-02 & WNT11 BMP4 PITX1 NOTCH3 ROR2 SIX2 TBX1 \\
\hline Glycoprotein biosynthetic process & 8 & 357 & $1.28 \mathrm{E}-02$ & HS3ST3A1 B3GNT4 FUT2 B3GNT8 B3GNT3 CHST8 ST8SIA2 ST6GAL2 \\
\hline $\begin{array}{l}\text { Transmembrane receptor protein serine/threo- } \\
\text { nine kinase signaling pathway }\end{array}$ & 8 & 358 & 1.30E-02 & BMP4 GREM1 FAM83G SMPD3 DLX5 RASL11B ROR2 SOX11 \\
\hline Axis specification & 4 & 86 & $1.30 \mathrm{E}-02$ & BMP4 SIX2 NRARP MDFI \\
\hline Negative regulation of $T$ cell differentiation & 3 & 41 & $1.30 \mathrm{E}-02$ & ZC3H12A NRARP BMP4 \\
\hline $\begin{array}{l}\text { Positive regulation of nitrogen compound } \\
\text { metabolic process }\end{array}$ & 35 & 3351 & $1.30 \mathrm{E}-02$ & $\begin{array}{l}\text { HGF DLX5 ZBTB16 BMP4 IL36G IL1RN HEYL JUP SOX11 FGF7 FGF18 } \\
\text { S100A9 ELF3 ZC3H12A GREM1 ADRB2 ALOX12B CCBE1 PITX1 NOTCH3 } \\
\text { WNT11 ALOX12 KRT17 CD36 CREB3L1 ROR2 SIX2 OVOL1 MAFB SH3RF3 } \\
\text { GLIS3 TBX1 SMPD3 NGF S100A12 }\end{array}$ \\
\hline Metanephric nephron development & 3 & 41 & 1.30E-02 & BMP4 GREM1 SIX2 \\
\hline Negative regulation of cell communication & 19 & 1440 & $1.31 \mathrm{E}-02$ & $\begin{array}{l}\text { SIK1 NRARP HGF MYOC BMP4 KLK14 IL1RN CREB3L1 HEYL GREM1 } \\
\text { ADRB2 FAM3D WNT11 RASL11B ZC3H12A AVPR1A ROR2 MDFI NOTCH3 }\end{array}$ \\
\hline Positive regulation of cellular metabolic process & 36 & 3482 & $1.31 \mathrm{E}-02$ & $\begin{array}{l}\text { HGF DLX5 ZBTB16 BMP4 IL36G IL1RN HEYL JUP SOX11 FGF7 FGF18 } \\
\text { S100A9 ELF3 ZC3H12A GREM1 ADRB2 ALOX12B CCBE1 PITX1 NOTCH3 } \\
\text { WNT11 ALOX12 KRT17 CD36 CREB3L1 AVPR1A ROR2 SIX2 OVOL1 MAFB } \\
\text { SH3RF3 GLIS3 TBX1 SMPD3 NGF S100A12 }\end{array}$ \\
\hline Negative regulation of signaling & 19 & 1444 & $1.35 \mathrm{E}-02$ & $\begin{array}{l}\text { SIK1 NRARP HGF MYOC BMP4 KLK14 IL1RN CREB3L1 HEYL GREM1 } \\
\text { ADRB2 FAM3D WNT11 RASL11B ZC3H12A AVPR1A ROR2 MDFI NOTCH3 }\end{array}$ \\
\hline Antimicrobial humoral response & 5 & 145 & $1.38 \mathrm{E}-02$ & SLPI S100A9 S100A12 DEFB4A PI3 \\
\hline Hair follicle development & 4 & 88 & $1.38 \mathrm{E}-02$ & KRT17 FGF7 TMEM79 KRT25 \\
\hline Regulation of epidermis development & 4 & 88 & $1.38 \mathrm{E}-02$ & BMP4 KRT17 TMEM79 SULT2B1 \\
\hline Artery development & 4 & 88 & $1.38 \mathrm{E}-02$ & BMP4 NOTCH3 WNT11 TBX1 \\
\hline Positive regulation of cell proliferation & 15 & 1022 & $1.39 \mathrm{E}-02$ & $\begin{array}{l}\text { NRARP BMP4 FGF7 GREM1 NOTCH3 SMPD3 DLX5 ALOX12 FGF18 } \\
\text { AVPR1A SIX2 SOX11 TGM1 TBX1 ADRA1D }\end{array}$ \\
\hline
\end{tabular}


Table 3 (continued)

\begin{tabular}{|c|c|c|c|c|}
\hline \multicolumn{5}{|l|}{ Down-regulated genes in alive $x$ dead } \\
\hline Functional category & Genes in list & Total genes & FDR & Genes \\
\hline Positive regulation of response to stimulus & 29 & 2621 & $1.39 \mathrm{E}-02$ & $\begin{array}{l}\text { BMP4 IL36G IL1RN RSPO3 NRARP JUP HGF MYOC KLK14 FGF18 S100A9 } \\
\text { S100A12 ZC3H12A ADRB2 ALOX12B CCBE1 DLX5 CHN1 CD36 GREM1 } \\
\text { ROR2 SOX11 DUOX1 WNT11 SH3RF3 NGF TBX1 FGF7 ELF3 }\end{array}$ \\
\hline Positive regulation of RNA metabolic process & 22 & 1789 & $1.39 E-02$ & $\begin{array}{l}\text { DLX5 ZBTB16 HEYL JUP SOX11 BMP4 ELF3 ZC3H12A PITX1 NOTCH3 } \\
\text { FGF7 CREB3L1 GREM1 ROR2 ADRB2 SIX2 OVOL1 MAFB WNT11 GLIS3 } \\
\text { TBX1 HGF }\end{array}$ \\
\hline Positive regulation of membrane depolarization & 2 & 12 & 1.39E-02 & MYOC ALOX12 \\
\hline Regulation of intracellular signal transduction & 24 & 2027 & $1.42 \mathrm{E}-02$ & $\begin{array}{l}\text { BMP4 IL36G IL1RN SIK1 HGF MYOC FGF18 S100A9 S100A12 ZC3H12A } \\
\text { ADRB2 ALOX12B CHN1 CD36 CREB3L1 GREM1 ROR2 SOX11 WNT11 } \\
\text { SH3RF3 NGF TBX1 FGF7 RHOD }\end{array}$ \\
\hline $\begin{array}{l}\text { Positive regulation of cellular protein metabolic } \\
\text { process }\end{array}$ & 21 & 1680 & $1.42 \mathrm{E}-02$ & $\begin{array}{l}\text { HGF BMP4 IL36G IL1RN FGF7 FGF18 S100A9 ZC3H12A ADRB2 ALOX12B } \\
\text { CCBE1 WNT11 ALOX12 KRT17 CD36 GREM1 ROR2 SH3RF3 TBX1 NGF } \\
\text { S100A12 }\end{array}$ \\
\hline Lung alveolus development & 3 & 43 & $1.42 \mathrm{E}-02$ & BMP4 SMPD3 PKDCC \\
\hline Pattern specification process & 9 & 451 & $1.42 \mathrm{E}-02$ & BMP4 ZBTB16 GREM1 ROR2 SIX2 NRARP MAFB MDFI TBX1 \\
\hline Growth & 15 & 1028 & $1.44 \mathrm{E}-02$ & $\begin{array}{l}\text { NGF BMP4 FGF7 CDA WNT11 BDKRB1 SMPD3 KRT17 CD36 CYP19A1 } \\
\text { PKDCC AVPR1A GREM1 ADRB2 S100A9 }\end{array}$ \\
\hline Molting cycle process & 4 & 90 & 1.44E-02 & KRT17 FGF7 TMEM79 KRT25 \\
\hline Hair cycle process & 4 & 90 & $1.44 \mathrm{E}-02$ & KRT17 FGF7 TMEM79 KRT25 \\
\hline Wnt signaling pathway & 10 & 543 & $1.48 \mathrm{E}-02$ & WNT11 RSPO3 NRARP JUP GREM1 DLX5 SDC1 ROR2 MYOC MDFI \\
\hline Negative regulation of osteoblast differentiation & 3 & 44 & 1.49E-02 & TWIST2 ID3 GREM1 \\
\hline Skin epidermis development & 4 & 91 & $1.49 \mathrm{E}-02$ & KRT17 FGF7 TMEM79 KRT25 \\
\hline Regulation of Wnt signaling pathway & 8 & 371 & $1.49 \mathrm{E}-02$ & RSPO3 NRARP JUP GREM1 WNT11 DLX5 ROR2 MDFI \\
\hline Cell-cell signaling by wnt & 10 & 545 & $1.50 E-02$ & WNT11 RSPO3 NRARP JUP GREM1 DLX5 SDC1 ROR2 MYOC MDFI \\
\hline Digestive system development & 5 & 151 & $1.54 \mathrm{E}-02$ & BMP4 PKDCC SIX2 SOX11 WNT11 \\
\hline Insemination & 2 & 13 & $1.57 E-02$ & KLK14 AVPR1A \\
\hline Extracellular structure organization & 9 & 460 & $1.57 \mathrm{E}-02$ & COL8A1 COL28A1 SMPD3 CD36 ADAMTSL4 ELF3 SDC1 GREM1 CREB3L1 \\
\hline Protein import into nucleus & 5 & 152 & $1.57 E-02$ & BMP4 ZC3H12A JUP CD36 SIX2 \\
\hline Positive regulation of cell death & 12 & 741 & $1.61 \mathrm{E}-02$ & $\begin{array}{l}\text { BMP4 ADAMTSL4 S100A9 ZC3H12A WNT11 ALOX12 ZBTB16 ID3 CD36 } \\
\text { SIK1 PNMA5 NGF }\end{array}$ \\
\hline Muscle structure development & 11 & 645 & $1.61 E-02$ & BMP4 USP2 PITX1 SDC1 ID3 HEYL AVPR1A GREM1 SOX11 SIK1 TBX1 \\
\hline Anterior/posterior pattern specification & 6 & 222 & 1.63E-02 & BMP4 ZBTB16 ROR2 SIX2 NRARP TBX1 \\
\hline $\begin{array}{l}\text { Epithelial cell differentiation involved in kidney } \\
\text { development }\end{array}$ & 3 & 46 & $1.63 \mathrm{E}-02$ & BMP4 GREM1 SIX2 \\
\hline Regulation of canonical Wnt signaling pathway & 7 & 298 & 1.65E-02 & RSPO3 NRARP JUP GREM1 WNT11 DLX5 ROR2 \\
\hline $\begin{array}{l}\text { Regulation of blood vessel endothelial cell } \\
\text { migration }\end{array}$ & 4 & 95 & $1.68 \mathrm{E}-02$ & ANGPT2 ALOX12 FGF18 JUP \\
\hline Secretion by cell & 21 & 1715 & $1.70 E-02$ & $\begin{array}{l}\text { CYP19A1 FGF7 FAM3D CHRNA4 SMPD3 CD36 IL1RN TMEM79 ZC3H12A } \\
\text { AVPR1A SOX11 SDC1 G6PC2 CREB3L1 HGF GLP1R SLPI CDA S100A9 } \\
\text { S100A12 JUP }\end{array}$ \\
\hline Gland development & 9 & 468 & $1.71 \mathrm{E}-02$ & HGF PITX1 BMP4 CYP19A1 FGF7 ELF3 MAFB WNT11 TBX1 \\
\hline Embryonic cranial skeleton morphogenesis & 3 & 47 & $1.71 \mathrm{E}-02$ & BMP4 SIX2 TBX1 \\
\hline Regulation of biomineral tissue development & 4 & 97 & $1.78 \mathrm{E}-02$ & BMP4 PKDCC ADRB2 GREM1 \\
\hline Mating & 3 & 48 & $1.80 \mathrm{E}-02$ & KLK14 PI3 AVPR1A \\
\hline Response to external stimulus & 28 & 2561 & $1.80 \mathrm{E}-02$ & $\begin{array}{l}\text { IL36G IL1RN SIK1 DEFB4A HGF ANGPT2 SLPI CD36 FGF7 FGF18 S100A9 } \\
\text { S100A12 ZC3H12A JUP AJAP1 USP2 WNT11 BDKRB1 DLX5 ALOX12 } \\
\text { BMP4 CHN1 CYP19A1 AVPR1A GREM1 ADRB2 PI3 LTB4R2 }\end{array}$ \\
\hline $\begin{array}{l}\text { Positive regulation of canonical Wnt signaling } \\
\text { pathway }\end{array}$ & 5 & 159 & 1.83E-02 & RSPO3 NRARP JUP DLX5 ROR2 \\
\hline $\begin{array}{l}\text { Positive regulation of protein modification } \\
\text { process }\end{array}$ & 17 & 1279 & $1.83 \mathrm{E}-02$ & $\begin{array}{l}\text { HGF BMP4 IL36G IL1RN FGF7 FGF18 ZC3H12A ADRB2 ALOX12B WNT11 } \\
\text { CD36 GREM1 ROR2 SH3RF3 TBX1 NGF S100A12 }\end{array}$ \\
\hline Somite development & 4 & 98 & $1.83 \mathrm{E}-02$ & WNT11 ROR2 SOX11 NRARP \\
\hline Regulation of binding & 8 & 388 & 1.83E-02 & ID3 SLPI BMP4 ADRB2 SOX11 CD36 MDFI NGF \\
\hline Genitalia development & 3 & 49 & $1.87 \mathrm{E}-02$ & CYP19A1 GREB1L ROR2 \\
\hline $\begin{array}{l}\text { Positive regulation of biomineral tissue develop- } \\
\text { ment }\end{array}$ & 3 & 49 & 1.87E-02 & BMP4 PKDCC ADRB2 \\
\hline
\end{tabular}


Table 3 (continued)

\begin{tabular}{|c|c|c|c|c|}
\hline \multicolumn{5}{|l|}{ Down-regulated genes in alive $x$ dead } \\
\hline Functional category & Genes in list & Total genes & FDR & Genes \\
\hline Ear development & 6 & 232 & $1.93 \mathrm{E}-02$ & DLX5 BMP4 ROR2 SIX2 MAFB TBX1 \\
\hline Branching morphogenesis of an epithelial tube & 5 & 162 & $1.94 \mathrm{E}-02$ & NRARP BMP4 GREB1L GREM1 SIX2 \\
\hline Positive regulation of exosomal secretion & 2 & 15 & 1.95E-02 & SDC1 SMPD3 \\
\hline Regulation of transcription by RNA polymerase II & 30 & 2830 & $1.95 \mathrm{E}-02$ & $\begin{array}{l}\text { DLX5 ZBTB16 BMP4 ELF3 HEYL JUP SOX11 ZC3H12A GREM1 FAM83G } \\
\text { PITX1 NOTCH3 SMPD3 GLIS3 ID3 TBX4 CD36 CREB3L1 ROR2 ADRB2 SIX2 } \\
\text { OVOL1 ZNF154 NRARP MAFB ZNF469 USP2 MDFI TBX1 HGF }\end{array}$ \\
\hline $\begin{array}{l}\text { Activation of transmembrane receptor protein } \\
\text { tyrosine kinase activity }\end{array}$ & 2 & 15 & $1.95 \mathrm{E}-02$ & GREM1 ADRB2 \\
\hline $\begin{array}{l}\text { Positive regulation of macromolecule biosyn- } \\
\text { thetic process }\end{array}$ & 23 & 1978 & $1.96 \mathrm{E}-02$ & $\begin{array}{l}\text { DLX5 ZBTB16 HEYL JUP SOX11 HGF BMP4 ELF3 ZC3H12A GREM1 PITX1 } \\
\text { NOTCH3 KRT17 FGF7 CREB3L1 ROR2 ADRB2 SIX2 OVOL1 MAFB WNT11 } \\
\text { GLIS3 TBX1 }\end{array}$ \\
\hline Secretion & 22 & 1861 & $1.96 \mathrm{E}-02$ & $\begin{array}{l}\text { CYP19A1 FGF7 FAM3D CHRNA4 SMPD3 CD36 IL1RN TMEM79 ZC3H12A } \\
\text { AVPR1A SOX11 SDC1 G6PC2 ALOX12B CREB3L1 HGF GLP1R SLPI CDA } \\
\text { S100A9 S100A12 JUP }\end{array}$ \\
\hline $\begin{array}{l}\text { Positive regulation of smooth muscle cell } \\
\text { proliferation }\end{array}$ & 4 & 101 & $1.97 \mathrm{E}-02$ & BMP4 NOTCH3 SMPD3 ALOX12 \\
\hline Negative regulation of hemopoiesis & 5 & 164 & $2.00 E-02$ & MAFB ZBTB16 ZC3H12A NRARP BMP4 \\
\hline Regulation of interleukin-6 production & 5 & 164 & $2.00 E-02$ & IL36G IL1RN ZC3H12A HGF CD36 \\
\hline Response to organic cyclic compound & 14 & 975 & $2.02 \mathrm{E}-02$ & $\begin{array}{l}\text { IL 1RN DUOX1 HEYL JUP ANGPT2 SLC8A3 SDC1 ID3 CD36 ZC3H12A } \\
\text { AVPR1A CLDN4 BMP4 CATSPERB }\end{array}$ \\
\hline Intermediate filament cytoskeleton organization & 3 & 51 & $2.02 \mathrm{E}-02$ & TCHH KRT17 KRT25 \\
\hline $\begin{array}{l}\text { Negative regulation of lymphocyte differentia- } \\
\text { tion }\end{array}$ & 3 & 51 & $2.02 \mathrm{E}-02$ & ZC3H12A NRARP BMP4 \\
\hline Negative regulation of cell differentiation & 12 & 774 & $2.08 \mathrm{E}-02$ & $\begin{array}{l}\text { ID3 BMP4 MAFB TWIST2 NOTCH3 ZBTB16 ZC3H12A SIX2 SOX11 NRARP } \\
\text { GREM1 TBX1 }\end{array}$ \\
\hline Regulation of signaling receptor activity & 11 & 676 & $2.08 \mathrm{E}-02$ & LYNX1 HGF BMP4 NGF IL36G IL1RN FGF7 FGF18 GREM1 FAM3D ADRB2 \\
\hline $\begin{array}{l}\text { Negative regulation of transcription by RNA } \\
\text { polymerase II }\end{array}$ & 13 & 876 & $2.08 \mathrm{E}-02$ & $\begin{array}{l}\text { BMP4 SOX11 ZBTB16 NOTCH3 ID3 CD36 CREB3L1 HEYL OVOL1 NRARP } \\
\text { USP2 GLIS3 MDFI }\end{array}$ \\
\hline Anterior/posterior axis specification & 3 & 52 & $2.10 \mathrm{E}-02$ & BMP4 SIX2 NRARP \\
\hline Positive regulation of cell-matrix adhesion & 3 & 52 & $2.10 \mathrm{E}-02$ & MYOC CD36 JUP \\
\hline Intermediate filament-based process & 3 & 52 & $2.10 \mathrm{E}-02$ & TCHH KRT17 KRT25 \\
\hline $\begin{array}{l}\text { Regulation of transcription from RNA polymer- } \\
\text { ase II promoter involved in heart development }\end{array}$ & 2 & 16 & $2.11 \mathrm{E}-02$ & BMP4 GREM1 \\
\hline Regulation of exosomal secretion & 2 & 16 & $2.11 \mathrm{E}-02$ & SDC1 SMPD3 \\
\hline $\begin{array}{l}\text { Cellular response to oxygen-containing } \\
\text { compound }\end{array}$ & 16 & 1199 & $2.13 E-02$ & $\begin{array}{l}\text { IL36G IL1RN HGF ZC3H12A JUP SLC8A3 CHRNA4 SMPD3 GLP1R ID3 } \\
\text { CD36 AVPR1A ADRB2 WNT11 TBX1 GNA15 }\end{array}$ \\
\hline Positive regulation of apoptotic process & 11 & 680 & $2.13 \mathrm{E}-02$ & $\begin{array}{l}\text { BMP4 ADAMTSL4 S100A9 WNT11 ALOX12 ZBTB16 ID3 ZC3H12A SIK1 } \\
\text { PNMA5 NGF }\end{array}$ \\
\hline Negative regulation of response to stimulus & 21 & 1764 & $2.13 \mathrm{E}-02$ & $\begin{array}{l}\text { SIK1 NRARP HGF MYOC ANGPT2 BMP4 KLK14 IL1RN CREB3L1 HEYL } \\
\text { GREM1 ADRB2 AJAP1 WNT11 ALOX12 RASL11B CYP19A1 ZC3H12A } \\
\text { ROR2 MDFI NOTCH3 }\end{array}$ \\
\hline Negative regulation of binding & 5 & 168 & $2.13 \mathrm{E}-02$ & ID3 SLPI ADRB2 SOX11 MDFI \\
\hline $\begin{array}{l}\text { Positive regulation of macromolecule metabolic } \\
\text { process }\end{array}$ & 35 & 3498 & 2.13E-02 & $\begin{array}{l}\text { HGF DLX5 ZBTB16 BMP4 IL36G IL1RN HEYL JUP SOX11 FGF7 FGF18 } \\
\text { S100A9 ELF3 ZC3H12A GREM1 ADRB2 ALOX12B CCBE1 PITX1 NOTCH3 } \\
\text { WNT11 ALOX12 KRT17 CD36 CREB3L1 ROR2 SIX2 OVOL1 MAFB NGF } \\
\text { SH3RF3 GLIS3 ACTG2 TBX1 S100A12 }\end{array}$ \\
\hline Muscle organ development & 8 & 404 & $2.15 \mathrm{E}-02$ & BMP4 USP2 PITX1 ID3 HEYL GREM1 SOX11 TBX1 \\
\hline Negative regulation of DNA binding & 3 & 53 & $2.16 \mathrm{E}-02$ & ID3 SOX11 MDFI \\
\hline Mesenchyme morphogenesis & 3 & 53 & $2.16 \mathrm{E}-02$ & WNT11 HEYL ACTG2 \\
\hline Regulation of phosphorylation & 20 & 1653 & $2.17 \mathrm{E}-02$ & $\begin{array}{l}\text { HGF BMP4 IL36G IL1RN MYOC FGF7 FGF18 ZC3H12A GREM1 ADRB2 } \\
\text { ALOX12B WNT11 BDKRB1 SMPD3 CD36 ROR2 SH3RF3 TBX1 NGF } \\
\text { S100A12 }\end{array}$ \\
\hline Positive regulation of programmed cell death & 11 & 686 & $2.23 \mathrm{E}-02$ & $\begin{array}{l}\text { BMP4 ADAMTSL4 S100A9 WNT11 ALOX12 ZBTB16 ID3 ZC3H12A SIK1 } \\
\text { PNMA5 NGF }\end{array}$ \\
\hline Unsaturated fatty acid biosynthetic process & 3 & 54 & $2.25 \mathrm{E}-02$ & ALOX12 AVPR1A ALOX12B \\
\hline Inner ear morphogenesis & 4 & 107 & $2.25 \mathrm{E}-02$ & DLX5 ROR2 MAFB TBX1 \\
\hline Regulation of striated muscle cell differentiation & 4 & 107 & $2.25 \mathrm{E}-02$ & GREM1 BMP4 SIK1 TBX1 \\
\hline
\end{tabular}


Table 3 (continued)

\begin{tabular}{|c|c|c|c|c|}
\hline \multicolumn{5}{|l|}{ Down-regulated genes in alive $x$ dead } \\
\hline Functional category & Genes in list & Total genes & FDR & Genes \\
\hline Negative regulation of signal transduction & 17 & 1321 & $2.27 \mathrm{E}-02$ & $\begin{array}{l}\text { SIK1 NRARP HGF MYOC BMP4 KLK14 IL1RN CREB3L1 HEYL GREM1 } \\
\text { ADRB2 WNT11 RASL11B ZC3H12A ROR2 MDFI NOTCH3 }\end{array}$ \\
\hline Lipoxygenase pathway & 2 & 17 & $2.27 E-02$ & ALOX12 ALOX12B \\
\hline Interleukin-6 production & 5 & 172 & $2.27 E-02$ & IL36G IL1RN ZC3H12A HGF CD36 \\
\hline Linoleic acid metabolic process & 2 & 17 & $2.27 \mathrm{E}-02$ & ALOX12 ALOX12B \\
\hline Pulmonary valve morphogenesis & 2 & 17 & 2.27E-02 & BMP4 HEYL \\
\hline Negative regulation of molecular function & 16 & 1216 & $2.33 \mathrm{E}-02$ & $\begin{array}{l}\text { ID3 HGF SLPI BMP4 ZC3H12A HEYL ADRB2 PI3 NGF SOX11 LYNX1 } \\
\text { SPINK9 COL28A1 MDFI SIK1 LTB4R2 }\end{array}$ \\
\hline Positive regulation of metabolic process & 37 & 3789 & $2.34 \mathrm{E}-02$ & $\begin{array}{l}\text { HGF DLX5 ZBTB16 BMP4 IL36G IL1RN HEYL JUP SOX11 FGF7 FGF18 } \\
\text { CREB3L1 S100A9 ELF3 ZC3H12A GREM1 ADRB2 ALOX12B CCBE1 PITX1 } \\
\text { NOTCH3 WNT11 ALOX12 KRT17 CD36 AVPR1A ROR2 SIX2 OVOL1 MAFB } \\
\text { NGF SH3RF3 GLIS3 ACTG2 TBX1 SMPD3 S100A12 }\end{array}$ \\
\hline Segmentation & 4 & 109 & $2.35 \mathrm{E}-02$ & BMP4 ROR2 NRARP MAFB \\
\hline Regulation of stress-activated MAPK cascade & 6 & 248 & $2.38 \mathrm{E}-02$ & IL36G IL1RN ZC3H12A HGF ROR2 SH3RF3 \\
\hline $\begin{array}{l}\text { Cell differentiation involved in kidney develop- } \\
\text { ment }\end{array}$ & 3 & 56 & $2.42 \mathrm{E}-02$ & BMP4 GREM1 SIX2 \\
\hline Regulation of epithelial cell proliferation & 7 & 330 & $2.43 E-02$ & NRARP BMP4 FGF7 DLX5 SOX11 TGM1 TBX1 \\
\hline Import into nucleus & 5 & 176 & $2.44 \mathrm{E}-02$ & BMP4 ZC3H12A JUP CD36 SIX2 \\
\hline $\begin{array}{l}\text { Regulation of stress-activated protein kinase } \\
\text { signaling cascade }\end{array}$ & 6 & 250 & $2.44 \mathrm{E}-02$ & IL36G IL1RN ZC3H12A HGF ROR2 SH3RF3 \\
\hline Positive regulation of protein metabolic process & 21 & 1796 & $2.45 \mathrm{E}-02$ & $\begin{array}{l}\text { HGF BMP4 IL36G IL1RN FGF7 FGF18 S100A9 ZC3H12A ADRB2 ALOX12B } \\
\text { CCBE1 WNT11 ALOX12 KRT17 CD36 GREM1 ROR2 SH3RF3 TBX1 NGF } \\
\text { S100A12 }\end{array}$ \\
\hline Carbohydrate derivative biosynthetic process & 12 & 800 & $2.45 \mathrm{E}-02$ & $\begin{array}{l}\text { HS3ST3A1 B3GNT4 FUT2 B3GNT8 B3GNT3 PAPSS2 CHST8 ST8SIA2 } \\
\text { SMPD3 ST6GAL2 SDC1 CDA }\end{array}$ \\
\hline Regulation of nucleocytoplasmic transport & 4 & 111 & $2.46 \mathrm{E}-02$ & BMP4 ZC3H12A JUP CD36 \\
\hline Embryonic digestive tract morphogenesis & 2 & 18 & $2.47 \mathrm{E}-02$ & SIX2 SOX11 \\
\hline Regulation of kidney development & 3 & 57 & $2.49 E-02$ & BMP4 GREM1 SIX2 \\
\hline Negative regulation of leukocyte differentiation & 4 & 112 & $2.52 \mathrm{E}-02$ & ZC3H12A NRARP MAFB BMP4 \\
\hline Lung morphogenesis & 3 & 58 & $2.60 E-02$ & BMP4 FGF7 SOX11 \\
\hline Leukocyte chemotaxis & 6 & 255 & $2.62 E-02$ & IL36G IL1RN S100A9 CYP19A1 GREM1 S100A12 \\
\hline Cell junction assembly & 6 & 255 & $2.62 \mathrm{E}-02$ & MYOC JUP WNT11 GREM1 RHOD FBLIM1 \\
\hline Mucopolysaccharide metabolic process & 4 & 114 & $2.65 E-02$ & HGF SMPD3 B3GNT4 B3GNT3 \\
\hline Regulation of growth & 11 & 710 & $2.67 \mathrm{E}-02$ & $\begin{array}{l}\text { NGF BMP4 CDA BDKRB1 KRT17 CD36 AVPR1A GREM1 ADRB2 WNT11 } \\
\text { S100A9 }\end{array}$ \\
\hline $\begin{array}{l}\text { Regulation of production of miRNAs involved in } \\
\text { gene silencing by miRNA }\end{array}$ & 2 & 19 & $2.68 \mathrm{E}-02$ & BMP4 ZC3H12A \\
\hline Exosomal secretion & 2 & 19 & $2.68 \mathrm{E}-02$ & SDC1 SMPD3 \\
\hline Keratinocyte migration & 2 & 19 & $2.68 \mathrm{E}-02$ & FGF7 LTB4R2 \\
\hline Kidney mesenchyme development & 2 & 19 & $2.68 \mathrm{E}-02$ & BMP4 SIX2 \\
\hline $\begin{array}{l}\text { Negative regulation of DNA-binding transcrip- } \\
\text { tion factor activity }\end{array}$ & 5 & 182 & $2.69 \mathrm{E}-02$ & ID3 ZC3H12A HEYL SIK1 MDFI \\
\hline Bone morphogenesis & 4 & 115 & $2.69 E-02$ & SMPD3 DLX5 BMP4 FGF18 \\
\hline Polysaccharide metabolic process & 4 & 116 & $2.76 \mathrm{E}-02$ & B3GNT4 B3GNT8 B3GNT3 SMPD3 \\
\hline Cellular oxidant detoxification & 4 & 117 & $2.83 \mathrm{E}-02$ & CD36 DUOX1 S100A9 GPX3 \\
\hline Molting cycle & 4 & 117 & $2.83 E-02$ & KRT25 KRT17 FGF7 TMEM79 \\
\hline Hair cycle & 4 & 117 & $2.83 E-02$ & KRT25 KRT17 FGF7 TMEM79 \\
\hline Extracellular exosome biogenesis & 2 & 20 & $2.84 \mathrm{E}-02$ & SDC1 SMPD3 \\
\hline $\begin{array}{l}\text { Positive regulation of cardiac muscle cell dif- } \\
\text { ferentiation }\end{array}$ & 2 & 20 & $2.84 \mathrm{E}-02$ & GREM1 BMP4 \\
\hline Regulation of cell growth & 8 & 432 & $2.84 \mathrm{E}-02$ & NGF CDA BDKRB1 KRT17 AVPR1A GREM1 WNT11 S100A9 \\
\hline Glycoprotein metabolic process & 8 & 432 & $2.84 \mathrm{E}-02$ & HS3ST3A1 B3GNT4 FUT2 B3GNT8 B3GNT3 CHST8 ST8SIA2 ST6GAL2 \\
\hline Notochord development & 2 & 20 & $2.84 \mathrm{E}-02$ & WNT11 ID3 \\
\hline $\begin{array}{l}\text { Blood vessel endothelial cell proliferation } \\
\text { involved in sprouting angiogenesis }\end{array}$ & 2 & 20 & $2.84 \mathrm{E}-02$ & NRARP BMP4 \\
\hline
\end{tabular}


Table 3 (continued)

\begin{tabular}{|c|c|c|c|c|}
\hline \multicolumn{5}{|l|}{ Down-regulated genes in alive $x$ dead } \\
\hline Functional category & Genes in list & Total genes & FDR & Genes \\
\hline $\begin{array}{l}\text { Positive regulation of stress-activated MAPK } \\
\text { cascade }\end{array}$ & 5 & 186 & $2.84 \mathrm{E}-02$ & IL36G IL1RN ZC3H12A ROR2 SH3RF3 \\
\hline Response to hydroperoxide & 2 & 20 & $2.84 \mathrm{E}-02$ & CD36 GPX3 \\
\hline Middle ear morphogenesis & 2 & 20 & $2.84 \mathrm{E}-02$ & $\mathrm{SIX} 2 \mathrm{TBX} 1$ \\
\hline Entrainment of circadian clock by photoperiod & 2 & 20 & 2.84E-02 & USP2 SIK1 \\
\hline Negative regulation of leukocyte chemotaxis & 2 & 20 & 2.84E-02 & CYP19A1 GREM1 \\
\hline $\begin{array}{l}\text { Regulation of production of small RNA involved } \\
\text { in gene silencing by RNA }\end{array}$ & 2 & 20 & $2.84 \mathrm{E}-02$ & BMP4 ZC3H12A \\
\hline Hormone secretion & 7 & 346 & $2.86 \mathrm{E}-02$ & CYP19A1 FAM3D SMPD3 IL1RN SOX11 G6PC2 GLP1R \\
\hline $\begin{array}{l}\text { Positive regulation of stress-activated protein } \\
\text { kinase signaling cascade }\end{array}$ & 5 & 187 & $2.88 \mathrm{E}-02$ & IL36G IL1RN ZC3H12A ROR2 SH3RF3 \\
\hline Negative regulation of endopeptidase activity & 6 & 264 & $2.89 \mathrm{E}-02$ & HGF PI3 SLPI SPINK9 COL28A1 NGF \\
\hline Neutrophil chemotaxis & 4 & 119 & $2.89 \mathrm{E}-02$ & IL36G IL1RN S100A9 S100A12 \\
\hline Embryonic digit morphogenesis & 3 & 62 & $2.91 \mathrm{E}-02$ & ZBTB16 BMP4 ROR2 \\
\hline $\begin{array}{l}\text { Modification of morphology or physiology of } \\
\text { other organism }\end{array}$ & 5 & 188 & $2.91 \mathrm{E}-02$ & SLPI S100A9 S100A12 ZC3H12A DEFB4A \\
\hline Positive regulation of cell-substrate adhesion & 4 & 120 & $2.95 \mathrm{E}-02$ & MYOC CD36 COL8A1 JUP \\
\hline $\begin{array}{l}\text { Positive regulation of protein serine/threonine } \\
\text { kinase activity }\end{array}$ & 7 & 349 & 2.95E-02 & BMP4 FGF18 HGF ROR2 ADRB2 NGF S100A12 \\
\hline Regulation of cell-substrate junction assembly & 3 & 63 & $3.00 \mathrm{E}-02$ & MYOC GREM1 RHOD \\
\hline Sialylation & 2 & 21 & $3.00 E-02$ & ST8SIA2 ST6GAL2 \\
\hline Regulation of protein localization to nucleus & 4 & 121 & $3.00 \mathrm{E}-02$ & BMP4 ZC3H12A JUP CD36 \\
\hline Protein glycosylation & 6 & 268 & $3.00 \mathrm{E}-02$ & B3GNT4 FUT2 B3GNT8 B3GNT3 ST8SIA2 ST6GAL2 \\
\hline Positive regulation of Wnt signaling pathway & 5 & 190 & $3.00 E-02$ & RSPO3 NRARP JUP DLX5 ROR2 \\
\hline $\begin{array}{l}\text { Negative regulation of chondrocyte differentia- } \\
\text { tion }\end{array}$ & 2 & 21 & $3.00 \mathrm{E}-02$ & BMP4 GREM1 \\
\hline Macromolecule glycosylation & 6 & 268 & $3.00 E-02$ & B3GNT4 FUT2 B3GNT8 B3GNT3 ST8SIA2 ST6GAL2 \\
\hline Regulation of focal adhesion assembly & 3 & 63 & $3.00 \mathrm{E}-02$ & MYOC GREM1 RHOD \\
\hline Regulation of mitochondrial depolarization & 2 & 21 & $3.00 \mathrm{E}-02$ & MYOC ALOX12 \\
\hline Pulmonary valve development & 2 & 21 & $3.00 E-02$ & BMP4 HEYL \\
\hline Chordate embryonic development & 10 & 632 & $3.02 \mathrm{E}-02$ & MDFI BMP4 RSPO3 ELF3 ROR2 SIX2 SOX11 NRARP WNT11 TBX1 \\
\hline O-glycan processing & 3 & 64 & 3.07E-02 & B3GNT4 B3GNT8 B3GNT3 \\
\hline Respiratory gaseous exchange & 3 & 65 & $3.18 \mathrm{E}-02$ & CHRNA4 CCBE1 MAFB \\
\hline Gland morphogenesis & 4 & 124 & $3.18 \mathrm{E}-02$ & HGF BMP4 FGF7 ELF3 \\
\hline Negative regulation of chemotaxis & 3 & 65 & $3.18 \mathrm{E}-02$ & ANGPT2 CYP19A1 GREM1 \\
\hline $\begin{array}{l}\text { Positive regulation of striated muscle cell dif- } \\
\text { ferentiation }\end{array}$ & 3 & 65 & $3.18 \mathrm{E}-02$ & GREM1 BMP4TBX1 \\
\hline Hormone transport & 7 & 357 & $3.19 \mathrm{E}-02$ & CYP19A1 FAM3D SMPD3 IL1RN SOX11 G6PC2 GLP1R \\
\hline Extracellular vesicle biogenesis & 2 & 22 & $3.21 \mathrm{E}-02$ & SDC1 SMPD3 \\
\hline $\begin{array}{l}\text { Positive regulation of transcription of Notch } \\
\text { receptor target }\end{array}$ & 2 & 22 & $3.21 \mathrm{E}-02$ & NOTCH3 HEYL \\
\hline Gastrulation & 5 & 195 & $3.21 \mathrm{E}-02$ & BMP4 IL1RN WNT11 SIX2 COL8A1 \\
\hline Negative regulation of peptidase activity & 6 & 273 & $3.21 \mathrm{E}-02$ & HGF PI3 SLPI NGF SPINK9 COL28A1 \\
\hline $\begin{array}{l}\text { Negative regulation of response to external } \\
\text { stimulus }\end{array}$ & 7 & 358 & $3.21 \mathrm{E}-02$ & ANGPT2 AJAP1 HGF ALOX12 CYP19A1 ZC3H12A GREM1 \\
\hline Proteolysis & 22 & 1988 & $3.24 \mathrm{E}-02$ & $\begin{array}{l}\text { CPXM2 HGF USP2 KLK14 S100A9 ZC3H12A CCBE1 KLK12 PRSS50 } \\
\text { ALOX12 PI3 SLPI KLK10 NGF ADAMTSL4 PRSS35 ADAMTS15 KLK13 } \\
\text { PRSS27 SPINK9 COL28A1 ADRB2 }\end{array}$ \\
\hline Negative regulation of cell-substrate adhesion & 3 & 66 & $3.25 E-02$ & MYOC AJAP1 ANGPT2 \\
\hline Transcription by RNA polymerase II & 30 & 2992 & $3.25 \mathrm{E}-02$ & $\begin{array}{l}\text { DLX5 ZBTB16 BMP4 ELF3 HEYL JUP SOX11 ZC3H12A GREM1 FAM83G } \\
\text { PITX1 NOTCH3 SMPD3 GLIS3 ID3 TBX4 CD36 CREB3L1 ROR2 ADRB2 SIX2 } \\
\text { OVOL1 ZNF154 NRARP MAFB ZNF469 USP2 MDFI TBX1 HGF }\end{array}$ \\
\hline Regulation of phosphate metabolic process & 21 & 1870 & $3.25 E-02$ & $\begin{array}{l}\text { HGF BMP4 IL36G IL1RN MYOC FGF7 FGF18 CDA ZC3H12A GREM1 } \\
\text { ADRB2 ALOX12B WNT11 BDKRB1 SMPD3 CD36 ROR2 SH3RF3 TBX1 NGF } \\
\text { S100A12 }\end{array}$ \\
\hline
\end{tabular}


Table 3 (continued)

\begin{tabular}{|c|c|c|c|c|}
\hline \multicolumn{5}{|l|}{ Down-regulated genes in alive $x$ dead } \\
\hline Functional category & Genes in list & Total genes & FDR & Genes \\
\hline Regulation of membrane potential & 8 & 450 & $3.25 \mathrm{E}-02$ & CHRNA4 KCNK12 MYOC SLC8A3 ALOX12 CD36 ADRB2 JUP \\
\hline Positive regulation of MAP kinase activity & 6 & 275 & $3.25 \mathrm{E}-02$ & BMP4 FGF18 HGF ROR2 NGF S100A12 \\
\hline Cellular detoxification & 4 & 126 & $3.26 \mathrm{E}-02$ & CD36 DUOX1 S100A9 GPX3 \\
\hline Carbohydrate metabolic process & 10 & 644 & $3.27 \mathrm{E}-02$ & $\begin{array}{l}\text { G6PC2 B3GNT4 B3GNT8 B3GNT3 ST8SIA2 ST6GAL2 SMPD3 CHST8 FUT2 } \\
\text { SIK1 }\end{array}$ \\
\hline $\begin{array}{l}\text { Positive regulation of nucleobase-containing } \\
\text { compound metabolic process }\end{array}$ & 22 & 1993 & $3.27 \mathrm{E}-02$ & $\begin{array}{l}\text { DLX5 ZBTB16 HEYL JUP SOX11 HGF BMP4 ELF3 ZC3H12A GREM1 PITX1 } \\
\text { NOTCH3 FGF7 CREB3L1 ROR2 ADRB2 SIX2 OVOL1 MAFB WNT11 GLIS3 } \\
\text { TBX1 }\end{array}$ \\
\hline Regulation of phosphorus metabolic process & 21 & 1872 & $3.27 \mathrm{E}-02$ & $\begin{array}{l}\text { HGF BMP4 IL36G IL1RN MYOC FGF7 FGF18 CDA ZC3H12A GREM1 } \\
\text { ADRB2 ALOX12B WNT11 BDKRB1 SMPD3 CD36 ROR2 SH3RF3 TBX1 NGF } \\
\text { S100A12 }\end{array}$ \\
\hline Protein localization to nucleus & 6 & 276 & $3.27 \mathrm{E}-02$ & BMP4 ZC3H12A JUP ZBTB16 CD36 SIX2 \\
\hline Cofactor catabolic process & 3 & 67 & $3.31 E-02$ & GPX3 DUOX1 ALDH1L1 \\
\hline Male genitalia development & 2 & 23 & $3.35 \mathrm{E}-02$ & GREB1L ROR2 \\
\hline Lens morphogenesis in camera-type eye & 2 & 23 & $3.35 \mathrm{E}-02$ & BMP4 SOX11 \\
\hline $\begin{array}{l}\text { Positive regulation of epidermal cell differentia- } \\
\text { tion }\end{array}$ & 2 & 23 & $3.35 \mathrm{E}-02$ & BMP4 SULT2B1 \\
\hline Negative regulation of muscle contraction & 2 & 23 & $3.35 \mathrm{E}-02$ & ADRB2 ZC $3 \mathrm{H} 12 \mathrm{~A}$ \\
\hline $\begin{array}{l}\text { Branching involved in salivary gland morpho- } \\
\text { genesis }\end{array}$ & 2 & 23 & $3.35 \mathrm{E}-02$ & HGF FGF7 \\
\hline Endothelial tube morphogenesis & 2 & 23 & $3.35 \mathrm{E}-02$ & BMP4 ALOX12 \\
\hline Morphogenesis of an endothelium & 2 & 23 & $3.35 \mathrm{E}-02$ & BMP4 ALOX12 \\
\hline Cranial skeletal system development & 3 & 68 & $3.39 E-02$ & BMP4 SIX2TBX1 \\
\hline Inflammatory response & 12 & 856 & $3.39 \mathrm{E}-02$ & $\begin{array}{l}\text { BDKRB1 LTB4R2 S100A9 HGF SDC1 IL36G IL1RN CYP19A1 S100A12 ELF3 } \\
\text { ZC3H12A CD36 }\end{array}$ \\
\hline Negative regulation of cell-cell adhesion & 5 & 200 & $3.39 \mathrm{E}-02$ & IL1RN ALOX12 ZC3H12A NRARP BMP4 \\
\hline Glycosylation & 6 & 280 & $3.41 \mathrm{E}-02$ & B3GNT4 FUT2 B3GNT8 B3GNT3 ST8SIA2 ST6GAL2 \\
\hline $\begin{array}{l}\text { Embryo development ending in birth or egg } \\
\text { hatching }\end{array}$ & 10 & 652 & $3.44 \mathrm{E}-02$ & MDFI BMP4 RSPO3 ELF3 ROR2 SIX2 SOX11 NRARP WNT11 TBX1 \\
\hline Regulation of adherens junction organization & 3 & 69 & $3.48 \mathrm{E}-02$ & MYOC GREM1 RHOD \\
\hline Skeletal muscle cell differentiation & 3 & 69 & $3.48 \mathrm{E}-02$ & HEYL SOX11 TBX1 \\
\hline Ceramide biosynthetic process & 3 & 69 & $3.48 \mathrm{E}-02$ & ST8SIA2 ALOX12B SMPD3 \\
\hline Response to toxic substance & 9 & 555 & $3.49 \mathrm{E}-02$ & CHRNA4 HGF SMPD3 SDC1 CD36 DUOX1 S100A9 ZC3H12A GPX3 \\
\hline Photoperiodism & 2 & 24 & $3.51 E-02$ & USP2 SIK1 \\
\hline Positive regulation of lipid storage & 2 & 24 & $3.51 \mathrm{E}-02$ & ZC3H12A CD36 \\
\hline $\begin{array}{l}\text { Purine ribonucleoside bisphosphate metabolic } \\
\text { process }\end{array}$ & 2 & 24 & $3.51 \mathrm{E}-02$ & PAPSS2 SULT2B1 \\
\hline Myelination & 4 & 131 & $3.51 \mathrm{E}-02$ & MALL HGF MYOC SLC8A3 \\
\hline Positive regulation of protein kinase activity & 9 & 557 & $3.51 \mathrm{E}-02$ & BMP4 FGF18 HGF WNT11 GREM1 ROR2 ADRB2 NGF S100A12 \\
\hline $\begin{array}{l}3 \text {-phosphoadenosine } 5 \text {-phosphosulfate } \\
\text { metabolic process }\end{array}$ & 2 & 24 & $3.51 \mathrm{E}-02$ & PAPSS2 SULT2B1 \\
\hline Mitochondrial depolarization & 2 & 24 & $3.51 \mathrm{E}-02$ & MYOC ALOX12 \\
\hline Uterus development & 2 & 24 & $3.51 \mathrm{E}-02$ & CYP19A1 GREB1L \\
\hline Regulation of cellular protein metabolic process & 28 & 2768 & $3.54 \mathrm{E}-02$ & $\begin{array}{l}\text { HGF BMP4 IL36G IL1RN MYOC FGF7 FGF18 S100A9 ZC3H12A GREM1 } \\
\text { ADRB2 ALOX12B CCBE1 WNT11 BDKRB1 SMPD3 ALOX12 PI3 SLPI KRT17 } \\
\text { NGF CD36 ROR2 SPINK9 COL28A1 SH3RF3 TBX1 S100A12 }\end{array}$ \\
\hline Formation of primary germ layer & 4 & 132 & $3.56 \mathrm{E}-02$ & BMP4 WNT11 SIX2 COL8A1 \\
\hline Endocrine system development & 4 & 132 & $3.56 \mathrm{E}-02$ & BMP4 PITX1 WNT11 TBX1 \\
\hline Regulation of DNA binding & 4 & 132 & $3.56 \mathrm{E}-02$ & ID3 SOX11 MDFI NGF \\
\hline Striated muscle cell differentiation & 6 & 285 & $3.56 \mathrm{E}-02$ & BMP4 AVPR1A GREM1 SDC1 SIK1 TBX1 \\
\hline Inner ear development & 5 & 205 & $3.59 E-02$ & DLX5 BMP4 ROR2 MAFB TBX1 \\
\hline Neutrophil migration & 4 & 133 & $3.62 \mathrm{E}-02$ & IL36G IL1RN S100A9 S100A12 \\
\hline
\end{tabular}


Table 4 Sensory neuron markers

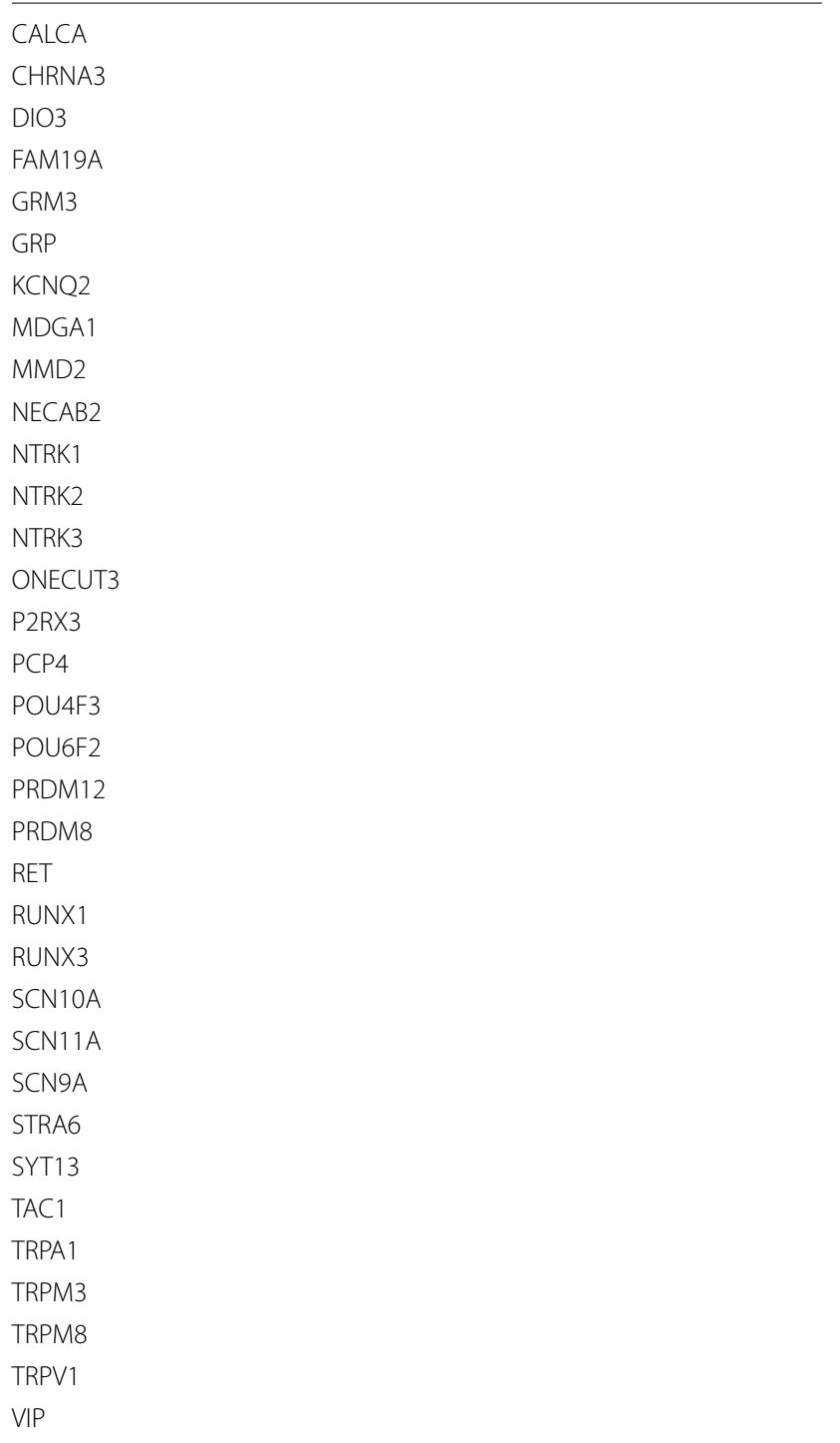

directly with several components within the tumor microenvironment, including cancer cells, immune cells, endothelial cells and others $[4,6,17,25,26,30$, $35,61,80,81,93,95,117,120,142,143,148]$. We detected an increase in intra-tumoral CGRP after overactivation of sensory neurons (Fig. 10). However, it remains unknown whether the decrease in the tumor size after sensory neurons overactivation is due to this increase in the intra-tumoral concentration of CGRP. Future studies will need to genetically eliminate specific receptors for this and other neuropeptides (such as receptor activity-modifying protein 1 (RAMP1) for CGRP) from different cellular components of the
Table 5 Angiogenesis-related genes down-regulated in alive $x$ dead

\begin{tabular}{lll}
\hline Gene.symbol & $\log 2 \mathrm{FC}$ & FDR \\
\hline ANGPT2 & -1.42767 & $3.23 \mathrm{E}-02$ \\
BMP4 & -1.23615 & $3.99 \mathrm{E}-02$ \\
CCBE1 & -1.04094 & $4.44 \mathrm{E}-02$ \\
COL8A1 & -1.22669 & $4.09 \mathrm{E}-02$ \\
CREB3L1 & -1.04632 & $4.44 \mathrm{E}-02$ \\
FGF18 & -1.35348 & $3.80 \mathrm{E}-03$ \\
GREM1 & -1.36182 & $1.10 \mathrm{E}-02$ \\
HGF & -1.19158 & $4.28 \mathrm{E}-03$ \\
JUP & -1.81687 & $1.10 \mathrm{E}-02$ \\
NOTCH3 & -1.22297 & $3.57 \mathrm{E}-03$ \\
NRARP & -1.42862 & $5.97 \mathrm{E}-03$ \\
RSPO3 & -1.48997 & $6.13 \mathrm{E}-04$ \\
TBX1 & -1.26122 & $3.69 \mathrm{E}-02$ \\
TBX4 & -1.84817 & $5.39 \mathrm{E}-03$ \\
ZC3H12A & -1.35742 & $4.67 \mathrm{E}-03$ \\
\hline
\end{tabular}

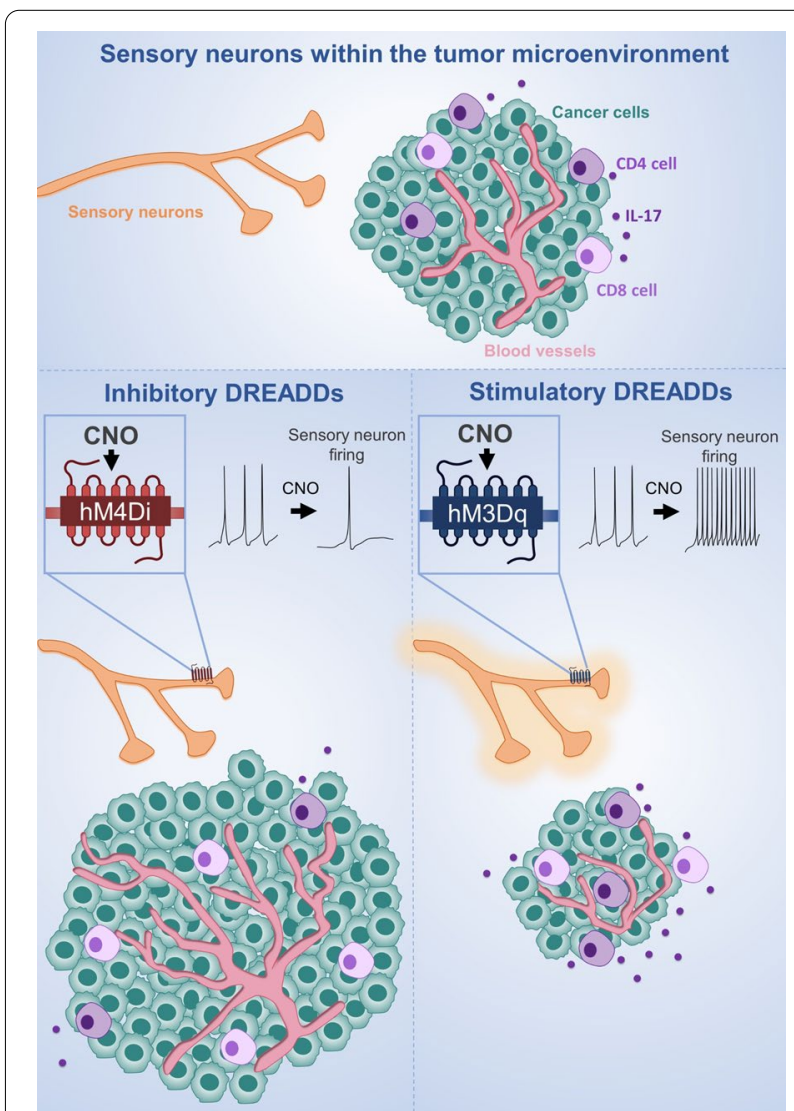

Fig. 9 Schematic illustration summarizing the results of sensory neurons' activity inhibition and overactivation in the melanoma microenvironment 
A

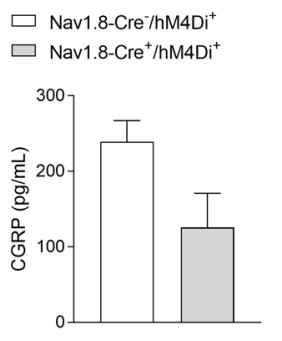

B $\square$ Nav1.8-Cre $/ \mathrm{hM}^{\mathrm{DDq}}{ }^{+}$

- Nav1.8-Cre ${ }^{+} / \mathrm{hM}^{2} \mathrm{Dq}^{+}$

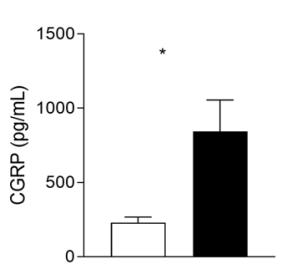

C

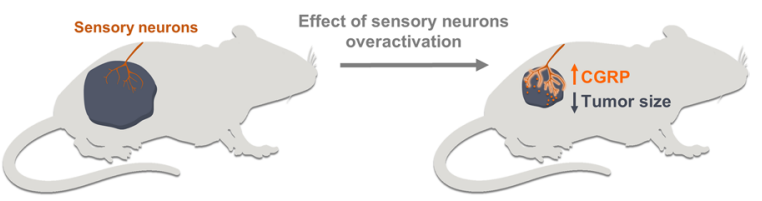

Fig. 10 Increase in sensory neurons activity leads to increase in intra-tumoral calcitonin gene-related peptide (CGRP). CGRP concentration was measured in tumor samples from Nav1.8-Cre + / hM4Di + and Nav1.8-Cre-/hM4Di + animals (A) and from Nav1.8-Cre +/hM3Dq + and Nav1.8-Cre-/hM3Dq + mice (B). (C) Schematic representation of the association between intra-tumoral concentration of CGRP and tumor size. $(n=5)$. Data are shown as mean \pm SEM

tumor microenvironment to reveal whether those communications are relevant for melanoma outcomes.

\section{Conclusion}

In conclusion, this work identifies sensory neurons overactivation as a potential strategy for blocking melanoma progression and improving patient outcomes. We also anticipate that drugs that reduce sensory neurons hyperexcitability used for analgesic treatment may cause undesired effects in cancer patients and need to be carefully evaluated in pre-clinical models of cancer before using them in these patients [34, 125]. Moreover, the relationship between sensory hyperexcitability and cancer outcomes is likely to inform studies of other cancers that are also infiltrated by sensory neurons.

\section{Supplementary Information}

The online version contains supplementary material available at https://doi. org/10.1186/s40478-021-01273-9.

Additional file 1. Gate strategy for regulatory markers. Representative contour plots showing proportion of CTLA-4 and PD-1 (top to bottom) in CD4+ and CD8+ (right to left) $T$ viable lymphocytes within CD45+ alive cells from tumor infiltrate.

Additional file 2. Interactions among genes related to angiogenesis which are overexpressed in SKCM patients presenting worse prognosis (dead vs. alive).

Additional file 3. Inferred proportion of immune infiltrated cells in SKCM patients from the TCGA cohort.
Additional file 4. Differences observed on inferred proportions of tumor-infiltrating CD4+ T cells, CD8 + T cells, dendritic cells and NK cells between samples of alive and dead SKCM patients from the TCGA cohort.

Additional file 5. Impact of Th17 immune response in melanoma. (A) Microarray analysis of skin samples from Melanoma $(n=46)$ and healthy $(n=16)$ individuals from GEO database: GSE15605 was analyzed by Phantasus [156] (https://genome.ifmo.ru/phantasus). Expression of Th17 immune response markers in melanoma samples, normalized to health samples, as Log2 Fold Change. (B) Survival curve from melanoma patients. The prognostic impact of IL17A expression in melanoma patients was evaluated using the R2: Genomics Analysis and Visualization Platform (http://r2.amc.nl). We evaluated the survival probability of patients with melanoma based on their tumor transcriptome $(n=214)$ [19]. High expression of IL17A in melanoma is correlated with increased patient survival. Differences were considered significant at $P$ value $<0.05$.

\section{Acknowledgements}

Alexander Birbrair is supported by a research productivity fellowship from Conselho Nacional de Desenvolvimento Científico e Tecnológico (CNPq-PQ2), a Grant from Instituto Serrapilheira/Serra-1708-15285, a Grant from Pró-reitoria de Pesquisa/Universidade Federal de Minas Gerais (PRPq/UFMG) (Edital 05/2016); a Grant from Fundação de Amparo à Pesquisa do Estado de Minas Gerais - FAPEMIG (Chamada N01/2021 - Demanda Universal, APQ-01321-

21), a Grant from FAPEMIG [Rede Mineira de Engenharia de Tecidos e Terapia Celular (REMETTEC, RED-00570-16)]; a Grant from FAPEMIG [Rede De Pesquisa Em Doenças Infecciosas Humanas E Animais Do Estado De Minas Gerais (RED-00313-16)]; and a Grant from MCTIC/CNPq No 28/2018 (Universal/Faixa A). Remo C. Russo is supported by a research productivity fellowship from Conselho Nacional de Desenvolvimento Científico e Tecnológico (CNPq-PQ2) and a Grant from FAPEMIG (Chamada N01/2021 - Demanda Universal). Akiva Mintz is supported by the National Institute of Health (1R01CA179072-01A1) and by the American Cancer Society Mentored Research Scholar Grant (124443-MRSG-13-121-01-CDD). Pedro A. C. Costa is supported by a postdoctoral fellowship (PNPD) from CAPES. Walison N. Silva is supported by master fellowships from CAPES. Caroline C. Picoli and Alinne C. Costa are supported by doctoral fellowships from CAPES. The authors also thank CAPI (UFMG) for microscopical technical support and Laboratory of Flow Cytometry at the Instituto de Ciências Biológicas/UFMG (http://labs.icb.ufmg.br/citometria/)" for providing the equipment and technical support for experiments involving flow cytometry.

\section{Availability of data and materials}

Data will be made available on reasonable request.

\section{Declarations}

\section{Competing interests}

The authors have no competing interests to declare.

\section{Author details}

${ }^{1}$ Departamento de Patologia, Universidade Federal de Minas Gerais, Belo Horizonte, MG, Brasil. ${ }^{2}$ Centro de Oncologia Molecular, Hospital Sirio-Libanes, Sao Paulo, SP, Brasil. ${ }^{3}$ Departamento de Bioquimica e Imunologia, Universidade Federal de Minas Gerais, Belo Horizonte, MG, Brasil. ${ }^{4}$ Departamento de Fisiologia e Biofísica, Universidade Federal de Minas Gerais, Belo Horizonte, MG, Brasil. ${ }^{5}$ Departamento de Farmacologia, Universidade Federal de Goiás, Goiânia, GO, Brasil. ${ }^{6}$ Centro das Ciências Biológicas e da Saúde, Universidade Federal do Oeste da Bahia, Barreiras, BA, Brasil. ' Departamento de Genetica, Ecologia e Evolucao, Universidade Federal de Minas Gerais, Belo Horizonte, MG, Brasil. ${ }^{8}$ Departamento de Farmacologia, Universidade de São Paulo, Ribeirão Preto, SP, Brasil. ${ }^{9}$ Department of Radiology, Columbia University Medical Center, New York, NY, USA.

Received: 27 July 2021 Accepted: 10 October 2021

Published online: 16 November 2021 


\section{References}

1. Allen JK, Armaiz-Pena GN, Nagaraja AS, Sadaoui NC, Ortiz T, Dood R, Ozcan M, Herder DM, Haemmerle M, Gharpure KM et al (2018) Sustained adrenergic signaling promotes intratumoral innervation through BDNF induction. Cancer Res 78:3233-3242. https://doi.org/10.1158/ 0008-5472.CAN-16-1701

2. Amaya F, Wang $H$, Costigan M, Allchorne AJ, Hatcher JP, Egerton J, Stean T, Morisset V, Grose D, Gunthorpe MJ et al (2006) The voltage-gated sodium channel $\mathrm{Na}(\mathrm{V}) 1.9$ is an effector of peripheral inflammatory pain hypersensitivity. J Neurosci 26:12852-12860. https://doi.org/10.1523/ JNEUROSCI.4015-06.2006

3. Ankathatti Munegowda M, Deng Y, Mulligan SJ, Xiang J (2011) Th17 and Th17-stimulated CD8(+) T cells play a distinct role in Th17-induced preventive and therapeutic antitumor immunity. Cancer Immunol Immunother 60:1473-1484. https://doi.org/10.1007/s00262-011-1054-y

4. Ansel JC, Brown JR, Payan DG, Brown MA (1993) Substance P selectively activates TNF-alpha gene expression in murine mast cells. J Immunol 150:4478-4485

5. Armbruster BN, Li X, Pausch MH, Herlitze S, Roth BL (2007) Evolving the lock to fit the key to create a family of $G$ protein-coupled receptors potently activated by an inert ligand. Proc Natl Acad Sci U S A 104:5163-5168. https://doi.org/10.1073/pnas.0700293104

6. Arslan Aydemir E, Simsek Oz E, Fidan Korcum A, Fiskin K (2011) Endostatin enhances radioresponse in breast cancer cells via alteration of substance P levels. Oncol Lett 2:879-886. https://doi.org/10.3892/ol. 2011.335

7. Ben-Shaanan TL, Schiller M, Azulay-Debby H, Korin B, Boshnak N, Koren T, Krot M, Shakya J, Rahat MA, Hakim F et al (2018) Modulation of antitumor immunity by the brain's reward system. Nat Commun 9:2723. https://doi.org/10.1038/s41467-018-05283-5

8. Bhatia A, Kumar Y (2011) Cancer-immune equilibrium: questions unanswered. Cancer Microenviron 4:209-217. https://doi.org/10.1007/ s12307-011-0065-8

9. Birbrair A, Zhang T, Wang ZM, Messi ML, Olson JD, Mintz A, Delbono O (2014) Type-2 pericytes participate in normal and tumoral angiogenesis. American J Physiology-Cell Physiol 307(1):C25-C38

10. Birbrair A, Sattiraju A, Zhu D, Zulato G, Batista I, Nguyen VT, Messi ML, Solingapuram Sai KK, Marini FC, Delbono O et al (2017) Novel peripherally derived neural-like stem cells as therapeutic carriers for treating glioblastomas. Stem Cells Transl Med 6:471-481. https://doi.org/10. 5966/sctm.2016-0007

11. Blank CU, Haining WN, Held W, Hogan PG, Kallies A, Lugli E, Lynn RC, Philip M, Rao A, Restifo NP et al (2019) Defining "T cell exhaustion." Nat Rev Immunol 19:665-674. https://doi.org/10.1038/s41577-019-0221-9

12. Bohn T, Rapp S, Luther N, Klein M, BruehI TJ, Kojima N, Aranda Lopez P, Hahlbrock J, Muth S, Endo S et al (2018) Tumor immunoevasion via acidosis-dependent induction of regulatory tumor-associated macrophages. Nat Immunol 19:1319-1329. https://doi.org/10.1038/ s41590-018-0226-8

13. Buch T, Heppner FL, Tertilt C, Heinen TJ, Kremer M, Wunderlich FT, Jung S, Waisman A (2005) A Cre-inducible diphtheria toxin receptor mediates cell lineage ablation after toxin administration. Nat Methods 2:419-426. https://doi.org/10.1038/nmeth762

14. Buonocore S, Ahern PP, Uhlig HH, Ivanov II, Littman DR, Maloy KJ, Powrie F (2010) Innate lymphoid cells drive interleukin-23-dependent innate intestinal pathology. Nature 464:1371-1375. https://doi.org/10 1038/nature08949

15. Catalano V, Turdo A, Di Franco S, Dieli F, Todaro M, Stassi G (2013) Tumor and its microenvironment: a synergistic interplay. Semin Cancer Biol 23:522-532. https://doi.org/10.1016/j.semcancer.2013.08.007

16. Chen H, Hu B, Lv X, Zhu S, Zhen G, Wan M, Jain A, Gao B, Chai Y, Yang $M$ et al (2019) Prostaglandin E2 mediates sensory nerve regulation of bone homeostasis. Nat Commun 10:181. https://doi.org/10.1038/ s41467-018-08097-7

17. Chen L, Yuan W, Chen Z, Wu S, Ge J, Chen J, Chen Z (2015) Vasoactive intestinal peptide represses activation of tumor-associated macrophages in gastric cancer via regulation of TNFalpha, IL-6, IL-12 and iNOS. Int J Oncol 47:1361-1370. https://doi.org/10.3892/ijo.2015.3126

18. Chiu IM, von Hehn CA, Woolf CJ (2012) Neurogenic inflammation and the peripheral nervous system in host defense and immunopathology. Nat Neurosci 15:1063-1067. https://doi.org/10.1038/nn.3144
19. Cirenajwis $H$, Ekedahl $H$, Lauss $M$, Harbst $K$, Carneiro A, Enoksson J, Rosengren F, Werner-Hartman L, Torngren T, Kvist A et al (2015) Molecular stratification of metastatic melanoma using gene expression profiling: Prediction of survival outcome and benefit from molecular targeted therapy. Oncotarget 6:12297-12309. https://doi.org/10.18632/ oncotarget.3655

20. Ciric B, El-behi M, Cabrera R, Zhang GX, Rostami A (2009) IL-23 drives pathogenic IL-17-producing CD8+ T cells. J Immunol 182:5296-5305. https://doi.org/10.4049/jimmunol.0900036

21. Coffelt SB, Wellenstein MD, de Visser KE (2016) Neutrophils in cancer: neutral no more. Nat Rev Cancer 16:431-446. https://doi.org/10.1038/ nrc.2016.52

22. Cohen JT, Miner TJ, Vezeridis MP (2020) Is the neutrophil-to-lymphocyte ratio a useful prognostic indicator in melanoma patients? Melanoma Manag. https://doi.org/10.2217/mmt-2020-0006

23. Coimbra-Campos LMC, Silva WN, Baltazar LM, Costa PAC, Prazeres PHDM, Picoli CC, Costa AC, Rocha BGS, Santos GSP, Oliveira FMS et al (2021) Circulating Nestin-GFP + cells participate in the pathogenesis of Paracoccidioides brasiliensis in the lungs. Stem Cell Rev Rep 5:1069

24. Cupedo T, Crellin NK, Papazian N, Rombouts EJ, Weijer K, Grogan JL, Fibbe WE, Cornelissen JJ, Spits H (2009) Human fetal lymphoid tissue-inducer cells are interleukin 17-producing precursors to RORC+ CD127+ natural killer-like cells. Nat Immunol 10:66-74. https://doi.org/ 10.1038/ni.1668

25. Cyphert JM, Kovarova M, Allen IC, Hartney JM, Murphy DL, Wess J, Koller $\mathrm{BH}$ (2009) Cooperation between mast cells and neurons is essential for antigen-mediated bronchoconstriction. J Immunol 182:7430-7439. https://doi.org/10.4049/jimmunol.0900039

26. Dalaklioglu S, Erin N (2016) Substance P prevents vascular endothelial dysfunction in metastatic breast carcinoma. Protein Pept Lett 23:952-957. https://doi.org/10.2174/0929866523666160902114459

27. Darvin P, Toor SM, Sasidharan Nair V, Elkord E (2018) Immune checkpoint inhibitors: recent progress and potential biomarkers. Exp Mol Med 50:1-11. https://doi.org/10.1038/s12276-018-0191-1

28. de Kleijn S, Langereis JD, Leentjens J, Kox M, Netea MG, Koenderman L, Ferwerda G, Pickkers P, Hermans PW (2013) IFN-gamma-stimulated neutrophils suppress lymphocyte proliferation through expression of PD-L1. PLoS ONE 8:e72249. https://doi.org/10.1371/journal.pone.00722 49

29. Desiderio S, Vermeiren S, Van Campenhout C, Kricha S, Malki E, Richts S, Fletcher EV, Vanwelden T, Schmidt BZ, Henningfeld KA et al (2019) Prdm12 directs nociceptive sensory neuron development by regulating the expression of the NGF receptor TrkA. Cell Rep 26(3522-3536):e3525. https://doi.org/10.1016/j.celrep.2019.02.097

30. Ding W, Stohl LL, Wagner JA, Granstein RD (2008) Calcitonin generelated peptide biases Langerhans cells toward Th2-type immunity. J Immunol 181:6020-6026. https://doi.org/10.4049/jimmunol.181.9.6020

31. Dos Santos FRC, Guardia GDA, Dos Santos FF, Ohara DT, Galante PAF (2021) Reboot: a straightforward approach to identify genes and splicing isoforms associated with cancer patient prognosis. NAR Cancer. https://doi.org/10.1093/narcan/zcab024

32. Dubeykovskaya Z, Si Y, Chen X, Worthley DL, Renz BW, Urbanska AM, Hayakawa Y, Xu T, Westphalen CB, Dubeykovskiy A et al (2016) Neural innervation stimulates splenic TFF2 to arrest myeloid cell expansion and cancer. Nat Commun 7:10517. https://doi.org/10.1038/ncomm s10517

33. Egger ME, Bower MR, Czyszczon IA, Farghaly H, Noyes RD, Reintgen DS, Martin RC 2nd, Scoggins CR, Stromberg AJ, McMasters KM (2014) Comparison of sentinel lymph node micrometastatic tumor burden measurements in melanoma. J Am Coll Surg 218:519-528. https://doi. org/10.1016/j.jamcollsurg.2013.12.014

34. Eijkelkamp N, Linley JE, Baker MD, Minett MS, Cregg R, Werdehausen R, Rugiero F, Wood JN (2012) Neurological perspectives on voltage-gated sodium channels. Brain 135:2585-2612. https://doi.org/10.1093/brain/ aws225

35. Erin N, Korcum AF, Tanriover G, Kale S, Demir N, Koksoy S (2015) Activation of neuroimmune pathways increases therapeutic effects of radiotherapy on poorly differentiated breast carcinoma. Brain Behav Immun 48:174-185. https://doi.org/10.1016/j.bbi.2015.02.024

36. Erin N, Zhao W, Bylander J, Chase G, Clawson G (2006) Capsaicininduced inactivation of sensory neurons promotes a more aggressive 
gene expression phenotype in breast cancer cells. Breast Cancer Res Treat 99:351-364. https://doi.org/10.1007/s10549-006-9219-7

37. Faure L, Wang Y, Kastriti ME, Fontanet $P$, Cheung KKY, Petitpre C, Wu $\mathrm{H}$, Sun LL, Runge K, Croci L et al (2020) Single cell RNA sequencing identifies early diversity of sensory neurons forming via bi-potential intermediates. Nat Commun 11:4175. https://doi.org/10.1038/ s41467-020-17929-4

38. Fridman WH, Pages F, Sautes-Fridman C, Galon J (2012) The immune contexture in human tumours: impact on clinical outcome. Nat Rev Cancer 12:298-306. https://doi.org/10.1038/nrc3245

39. Gabrilovich DI (2017) Myeloid-derived suppressor cells. Cancer Immunol Res 5:3-8. https://doi.org/10.1158/2326-6066.CIR-16-0297

40. Gast CE, Silk AD, Zarour L, Riegler L, Burkhart JG, Gustafson KT, Parappilly MS, Roh-Johnson M, Goodman JR, Olson B, al (2018) Cell fusion potentiates tumor heterogeneity and reveals circulating hybrid cells that correlate with stage and survival. Sci Adv. https://doi.org/10.1126/ sciadv.aat7828

41. Gautron L, Sakata I, Udit S, Zigman JM, Wood JN, Elmquist JK (2011) Genetic tracing of Nav1.8-expressing vagal afferents in the mouse. $J$ Comp Neurol 519:3085-3101. https://doi.org/10.1002/cne.22667

42. Ge SX, Jung D, Yao R (2020) ShinyGO: a graphical gene-set enrichment tool for animals and plants. Bioinformatics 36:2628-2629. https://doi. org/10.1093/bioinformatics/btz931

43. Gerber AL, Munst A, Schlapbach C, Shafighi M, Kiermeir D, Husler R, Hunger RE (2014) High expression of FOXP3 in primary melanoma is associated with tumour progression. Br J Dermatol 170:103-109. https://doi.org/10.1111/bjd.12641

44. Gerdes J, Lemke H, Baisch H, Wacker HH, Schwab U, Stein H (1984) Cell cycle analysis of a cell proliferation-associated human nuclear antigen defined by the monoclonal antibody Ki-67. J Immunol 133:1710-1715

45. Giraldo NA, Sanchez-Salas R, Peske JD, Vano Y, Becht E, Petitprez F, Validire P, Ingels A, Cathelineau X, Fridman WH et al (2019) The clinical role of the TME in solid cancer. Br J Cancer 120:45-53. https://doi.org/ 10.1038/s41416-018-0327-z

46. Girardi M, Oppenheim DE, Steele CR, Lewis JM, Glusac E, Filler R, Hobby P, Sutton B, Tigelaar RE, Hayday AC (2001) Regulation of cutaneous malignancy by gammadelta T cells. Science 294:605-609. https://doi, org/10.1126/science.1063916

47. Gooden MJ, de Bock GH, Leffers N, Daemen T, Nijman HW (2011) The prognostic influence of tumour-infiltrating lymphocytes in cancer: a systematic review with meta-analysis. Br J Cancer 105:93-103. https:// doi.org/10.1038/bjc.2011.189

48. Gray-Schopfer V, Wellbrock C, Marais R (2007) Melanoma biology and new targeted therapy. Nature 445:851-857. https://doi.org/10.1038/ nature05661

49. Greten FR, Grivennikov SI (2019) Inflammation and cancer: triggers, mechanisms, and consequences. Immunity 51:27-41. https://doi.org/ 10.1016/j.immuni.2019.06.025

50. Grivennikov SI, Greten FR, Karin M (2010) Immunity, inflammation, and cancer. Cell 140:883-899. https://doi.org/10.1016/j.cell.2010.01.025

51. Guimaraes MZP, De Vecchi R, Vitoria G, Sochacki JK, Paulsen BS, Lima I, Rodrigues da Silva F, da Costa RFM, Castro NG, Breton L et al (2018) Generation of iPSC-derived human peripheral sensory neurons releasing substance P elicited by TRPV1 agonists. Front Mol Neurosci 11:277. https://doi.org/10.3389/fnmol.2018.00277

52. Gurzu S, Beleaua MA, Jung I (2018) The role of tumor microenvironment in development and progression of malignant melanomas-a systematic review. Rom J Morphol Embryol 59:23-28

53. Hamada H, Garcia-Hernandez Mde L, Reome JB, Misra SK, Strutt TM, McKinstry KK, Cooper AM, Swain SL, Dutton RW (2009) Tc17, a unique subset of CD8T cells that can protect against lethal influenza challenge. J Immunol 182:3469-3481. https://doi.org/10.4049/jimmunol.0801814

54. Hanahan D, Weinberg RA (2011) Hallmarks of cancer: the next generation. Cell 144:646-674. https://doi.org/10.1016/j.cell.2011.02.013

55. Hargadon KM, Johnson CE, Williams CJ (2018) Immune checkpoint blockade therapy for cancer: an overview of FDA-approved immune checkpoint inhibitors. Int Immunopharmacol 62:29-39. https://doi.org/ 10.1016/j.intimp.2018.06.001

56. Harrington LE, Hatton RD, Mangan PR, Turner H, Murphy TL, Murphy KM, Weaver CT (2005) Interleukin 17-producing CD4+ effector T cells develop via a lineage distinct from the Thelper type 1 and 2 lineages. Nat Immunol 6:1123-1132. https://doi.org/10.1038/ni1254

57. Hayakawa Y, Sakitani K, Konishi M, Asfaha S, Niikura R, Tomita H, Renz BW, Tailor Y, Macchini M, Middelhoff M et al (2017) Nerve growth factor promotes gastric tumorigenesis through aberrant cholinergic signaling. Cancer Cell 31:21-34. https://doi.org/10.1016/j.ccell.2016.11.005

58. He W, Hao J, Dong S, Gao Y, Tao J, Chi H, Flavell R, O'Brien RL, Born WK, Craft J et al (2010) Naturally activated $V$ gamma 4 gamma delta $T$ cells play a protective role in tumor immunity through expression of eomesodermin. J Immunol 185:126-133. https://doi.org/10.4049/ jimmunol.0903767

59. Hodi FS, O'Day SJ, McDermott DF, Weber RW, Sosman JA, Haanen JB, Gonzalez R, Robert C, Schadendorf D, Hassel JC et al (2010) Improved survival with ipilimumab in patients with metastatic melanoma. N Engl J Med 363:711-723. https://doi.org/10.1056/NEJMoa1003466

60. Hondermarck H, Jobling P (2018) The sympathetic nervous system drives tumor angiogenesis. Trends Cancer 4:93-94. https://doi.org/10. 1016/j.trecan.2017.11.008

61. Hosoi J, Murphy GF, Egan CL, Lerner EA, Grabbe S, Asahina A, Granstein RD (1993) Regulation of langerhans cell function by nerves containing calcitonin gene-related peptide. Nature 363:159-163. https://doi.org/ 10.1038/363159a0

62. Hu B, Lv X, Chen H, Xue P, Gao B, Wang X, Zhen G, Crane JL, Pan D, Liu S et al (2020) Sensory nerves regulate mesenchymal stromal cell lineage commitment by tuning sympathetic tones. J Clin Invest 130:3483-3498 https://doi.org/10.1172/JCl131554

63. Hu X, Majchrzak K, Liu X, Wyatt MM, Spooner CJ, Moisan J, Zou W, Carter LL, Paulos CM (2018) In Vitro priming of adoptively transferred T cells with a RORgamma agonist confers durable memory and stemness In Vivo. Cancer Res 78:3888-3898. https://doi.org/10.1158/0008-5472. CAN-17-3973

64. Huntington ND, Vosshenrich CA, Di Santo JP (2007) Developmental pathways that generate natural-killer-cell diversity in mice and humans. Nat Rev Immunol 7:703-714. https://doi.org/10.1038/nri2154

65. Jablonska J, Leschner S, Westphal K, Lienenklaus S, Weiss S (2010) Neutrophils responsive to endogenous IFN-beta regulate tumor angiogenesis and growth in a mouse tumor model. J Clin Invest 120:1151-1164. https://doi.org/10.1172/JCl37223

66. Jacobs JF, Nierkens S, Figdor CG, de Vries IJ, Adema GJ (2012) Regulatory $T$ cells in melanoma: the final hurdle towards effective immunotherapy? Lancet Oncol 13:e32-42. https://doi.org/10.1016/S1470-2045(11) 70155-3

67. Jang JH, Kim DH, Surh YJ (2021) Dynamic roles of inflammasomes in inflammatory tumor microenvironment. NPJ Precis Oncol 5:18. https:// doi.org/10.1038/s41698-021-00154-7

68. Jayaraj ND, Bhattacharyya BJ, Belmadani AA, Ren D, Rathwell CA, Hackelberg S, Hopkins BE, Gupta HR, Miller RJ, Menichella DM (2018) Reducing CXCR4-mediated nociceptor hyperexcitability reverses painful diabetic neuropathy. J Clin Invest 128:2205-2225. https://doi.org/10. $1172 / J C 192117$

69. Jensen HK, Donskov F, Marcussen $N$, Nordsmark M, Lundbeck F, von der Maase $\mathrm{H}$ (2009) Presence of intratumoral neutrophils is an independent prognostic factor in localized renal cell carcinoma. J Clin Oncol 27:4709-4717. https://doi.org/10.1200/JCO.2008.18.9498

70. Jesus ICG, Araújo FM, Mesquita T et al (2021) Molecular basis of Period 1 regulation by adrenergic signaling in the heart. FASEB J. 35:e21886. https://doi.org/10.1096/fi.202100441R

71. Kamiya A, Hayama Y, Kato S, Shimomura A, Shimomura T, Irie K, Kaneko R, Yanagawa Y, Kobayashi K, Ochiya T (2019) Genetic manipulation of autonomic nerve fiber innervation and activity and its effect on breast cancer progression. Nat Neurosci 22:1289-1305. https://doi.org/10. 1038/s41593-019-0430-3

72. Klages K, Mayer CT, Lahl K, Loddenkemper C, Teng MW, Ngiow SF, Smyth MJ, Hamann A, Huehn J, Sparwasser T (2010) Selective depletion of Foxp3+ regulatory $T$ cells improves effective therapeutic vaccination against established melanoma. Cancer Res 70:7788-7799. https://doi. org/10.1158/0008-5472.CAN-10-1736

73. Kobayashi N, Hiraoka N, Yamagami W, Ojima H, Kanai Y, Kosuge T, Nakajima A, Hirohashi S (2007) FOXP3+ regulatory T cells affect the development and progression of hepatocarcinogenesis. Clin Cancer Res 13:902-911. https://doi.org/10.1158/1078-0432.CCR-06-2363 
74. Kollgaard T, Ugurel-Becker S, Idorn M, Andersen MH, Becker JC, Straten PT (2015) Pre-vaccination frequencies of Th17 cells correlate with vaccine-induced T-cell responses to survivin-derived peptide epitopes. PLOS ONE 10:e0131934. https://doi.org/10.1371/journal.pone.0131934

75. Kryczek I, Banerjee M, Cheng P, Vatan L, Szeliga W, Wei S, Huang E, Finlayson E, Simeone D, Welling TH et al (2009) Phenotype, distribution, generation, and functional and clinical relevance of Th17 cells in the human tumor environments. Blood 114:1141-1149. https://doi.org/10. 1182/blood-2009-03-208249

76. Ladstein RG, Bachmann IM, Straume O, Akslen LA (2010) Ki-67 expression is superior to mitotic count and novel proliferation markers $\mathrm{PHH}$, MCM4 and mitosin as a prognostic factor in thick cutaneous melanoma. BMC Cancer 10:140. https://doi.org/10.1186/1471-2407-10-140

77. Langrish $C L$, Chen Y, Blumenschein WM, Mattson J, Basham B, Sedgwick JD, McClanahan T, Kastelein RA, Cua DJ (2005) IL-23 drives a pathogenic $T$ cell population that induces autoimmune inflammation. J Exp Med 201:233-240. https://doi.org/10.1084/jem.20041257

78. Leclerc M, Voilin E, Gros G et al (2019) Regulation of antitumour CD8 T-cell immunity and checkpoint blockade immunotherapy by Neuropilin-1. Nat Commun 10:3345. https://doi.org/10.1038/ s41467-019-11280-z

79. Lesokhin AM, Hohl TM, Kitano S, Cortez C, Hirschhorn-Cymerman D, Avogadri F, Rizzuto GA, Lazarus JJ, Pamer EG, Houghton AN et a (2012) Monocytic CCR2(+) myeloid-derived suppressor cells promote immune escape by limiting activated CD8 T-cell infiltration into the tumor microenvironment. Cancer Res 72:876-886. https://doi.org/10. 1158/0008-5472.CAN-11-1792

80. Levine JD, Clark R, Devor M, Helms C, Moskowitz MA, Basbaum Al (1984) Intraneuronal substance $P$ contributes to the severity of experimental arthritis. Science 226:547-549. https://doi.org/10.1126/science.6208609

81. Li GH, Qian W, Song GQ, Hou XH (2007) Effect of vasoactive intestinal peptide on gastric adenocarcinoma. J Gastroenterol Hepatol 22:1328-1335. https://doi.org/10.1111/j.1440-1746.2007.04947.x

82. Li L, Rutlin M, Abraira VE, Cassidy C, Kus L, Gong S, Jankowski MP, Luo W, Heintz N, Koerber HR et al (2011) The functional organization of cutaneous low-threshold mechanosensory neurons. Cell 147:1615-1627. https://doi.org/10.1016/j.cell.2011.11.027

83. Li X, Kostareli E, Suffner J, Garbi N, Hammerling GJ (2010) Efficient Treg depletion induces T-cell infiltration and rejection of large tumors. Eur J Immunol 40:3325-3335. https://doi.org/10.1002/eji.201041093

84. Liebig C, Ayala G, Wilks JA, Berger DH, Albo D (2009) Perineural invasion in cancer: a review of the literature. Cancer 115:3379-3391. https://doi. org/10.1002/cncr.24396

85. Liu J, Lichtenberg T, Hoadley KA, Poisson LM, Lazar AJ, Cherniack AD, Kovatich AJ, Benz CC, Levine DA, Lee AV et al (2018) An integrated TCGA pan-cancer clinical data resource to drive high-quality survival outcome analytics. Cell 173(400-416):e411. https://doi.org/10.1016/j. cell.2018.02.052

86. Lu P, Weaver VM, Werb Z (2012) The extracellular matrix: a dynamic niche in cancer progression. J Cell Biol 196:395-406. https://doi.org/10. 1083/jcb.201102147

87. Lund AW, Duraes FV, Hirosue S, Raghavan VR, Nembrini C, Thomas SN, Issa A, Hugues S, Swartz MA (2012) VEGF-C promotes immune tolerance in B16 melanomas and cross-presentation of tumor antigen by lymph node lymphatics. Cell Rep 1:191-199. https://doi.org/10.1016/j. celrep.2012.01.005

88. Maacha S, Bhat AA, Jimenez L, Raza A, Haris M, Uddin S, Grivel JC (2019) Extracellular vesicles-mediated intercellular communication: roles in the tumor microenvironment and anti-cancer drug resistance. Mol Cancer 18:55. https://doi.org/10.1186/s12943-019-0965-7

89. Magnon C, Hall SJ, Lin J, Xue X, Gerber L, Freedland SJ, Frenette PS (2013) Autonomic nerve development contributes to prostate cancer progression. Science 341:1236361. https://doi.org/10.1126/science. 1236361

90. Marciante AB, Farmer GE, Cunningham JT (2020) Gq DREADD activation of CaMKIla MnPO neurons stimulates nitric oxide activity. J Neurophysiol 124:591-609. https://doi.org/10.1152/jn.00239.2020

91. Martin-Orozco N, Muranski P, Chung Y, Yang XO, Yamazaki T, Lu S, Hwu P, Restifo NP, Overwijk WW, Dong C (2009) T helper 17 cells promote cytotoxic T cell activation in tumor immunity. Immunity 31:787-798. https://doi.org/10.1016/j.immuni.2009.09.014
92. Matsuo K, Itoh T, Koyama A, Imamura R, Kawai S, Nishiwaki K, Oiso N, Kawada A, Yoshie O, Nakayama T (2016) CCR4 is critically involved in effective antitumor immunity in mice bearing intradermal B16 melanoma. Cancer Lett 378:16-22. https://doi.org/10.1016/j.canlet.2016.04. 039

93. Mayordomo C, Garcia-Recio S, Ametller E, Fernandez-Nogueira P, PastorArroyo EM, Vinyals L, Casas I, Gascon P, Almendro V (2012) Targeting of substance $P$ induces cancer cell death and decreases the steady state of EGFR and Her2. J Cell Physiol 227:1358-1366. https://doi.org/10. 1002/jcp.22848

94. Michel ML, Keller AC, Paget C, Fujio M, Trottein F, Savage PB, Wong CH, Schneider E, Dy M, Leite-de-Moraes MC (2007) Identification of an IL-17-producing NK1.1(neg) iNKT cell population involved in airway neutrophilia. J Exp Med 204:995-1001. https://doi.org/10.1084/jem. 20061551

95. Mikami N, Matsushita H, Kato T, Kawasaki R, Sawazaki T, Kishimoto T, Ogitani Y, Watanabe K, Miyagi Y, Sueda Ket al (2011) Calcitonin generelated peptide is an important regulator of cutaneous immunity: effect on dendritic cell and T cell functions. J Immunol 186:6886-6893. https://doi.org/10.4049/jimmunol.1100028

96. Mittal D, Gubin MM, Schreiber RD, Smyth MJ (2014) New insights into cancer immunoediting and its three component phases-elimination, equilibrium and escape. Curr Opin Immunol 27:16-25. https://doi.org/ 10.1016/j.coi.2014.01.004

97. Mu W, Rana S, Zoller M (2013) Host matrix modulation by tumor exosomes promotes motility and invasiveness. Neoplasia 15:875-887. https://doi.org/10.1593/neo.13786

98. Mukherji B (2013) Immunology of melanoma. Clin Dermatol 31:156165. https://doi.org/10.1016/j.clindermatol.2012.08.017

99. Muranski P, Boni A, Antony PA, Cassard L, Irvine KR, Kaiser A, Paulos CM, Palmer DC, Touloukian CE, Ptak K et al (2008) Tumor-specific Th17-polarized cells eradicate large established melanoma. Blood 112:362-373. https://doi.org/10.1182/blood-2007-11-120998

100. Muranski P, Borman ZA, Kerkar SP, Klebanoff CA, Ji Y, Sanchez-Perez L, Sukumar M, Reger RN, Yu Z, Kern SJ et al (2011) Th17 cells are long lived and retain a stem cell-like molecular signature. Immunity 35:972-985. https://doi.org/10.1016/j.immuni.2011.09.019

101. Navarro X, Krueger TB, Lago N, Micera S, Stieglitz T, Dario P (2005) A critical review of interfaces with the peripheral nervous system for the control of neuroprostheses and hybrid bionic systems. J Peripher Nerv Syst 10:229-258. https://doi.org/10.1111/j.1085-9489.2005.10303.x

102. Newman AM, Steen CB, Liu CL, Gentles AJ, Chaudhuri AA, Scherer F, Khodadoust MS, Esfahani MS, Luca BA, Steiner D et al (2019) Determining cell type abundance and expression from bulk tissues with digital cytometry. Nat Biotechnol 37:773-782. https://doi.org/10.1038/ s41587-019-0114-2

103. Papotto PH, Ribot JC, Silva-Santos B (2017) IL-17(+) gammadelta T cells as kick-starters of inflammation. Nat Immunol 18:604-611. https://doi. org/10.1038/ni.3726

104. Passarelli A, Mannavola F, Stucci LS, Tucci M, Silvestris F (2017) Immune system and melanoma biology: a balance between immunosurveillance and immune escape. Oncotarget 8:106132-106142. https://doi. org/10.18632/oncotarget.22190

105. Picoli CC, Costa AC, Rocha BGS, Silva WN, Santos GSP, Prazeres P, Costa PAC, Oropeza A, da Silva RA, Azevedo VAC et al (2020) Sensory nerves in the spotlight of the stem cell niche. Stem Cells Transl Med. https://doi. org/10.1002/sctm.20-0284

106. Picoli CC, Goncalves BOP, Santos GSP, Rocha BGS, Costa AC, Resende RR, Birbrair A (2021) Pericytes cross-talks within the tumor microenvironment. Biochim Biophys Acta Rev Cancer 1876:188608. https://doi.org/ 10.1016/j.bbcan.2021.188608

107. Pillay J, Tak T, Kamp VM, Koenderman L (2013) Immune suppression by neutrophils and granulocytic myeloid-derived suppressor cells: similarities and differences. Cell Mol Life Sci 70:3813-3827. https://doi.org/10. 1007/s00018-013-1286-4

108. Prazeres $P$, Leonel C, Silva WN, Rocha BGS, Santos GSP, Costa AC, Picoli CC, Sena IFG, Goncalves WA, Vieira MS et al (2020) Ablation of sensory nerves favours melanoma progression. J Cell Mol Med. https://doi.org/ 10.1111/jcmm.15381

109. Prevost-Blondel A, Neuenhahn M, Rawiel M, Pircher H (2000) Differential requirement of perforin and IFN-gamma in CD8 T cell-mediated 
immune responses against B16.F10 melanoma cells expressing a viral antigen. Eur J Immunol 30:2507-2515. https://doi.org/10.1002/15214141(200009)30:9\%3c2507::AID-IMMU2507\%3e3.0.CO;2-V

110. Qiao LY, Madar J (2021) An objective approach to assess colonic pain in mice using colonometry. PLOS ONE 16:e0245410. https://doi.org/10 1371/journal.pone.0245410

111. Quaresmini D, Guida M (2020) Neoangiogenesis in melanoma: an issue in biology and systemic treatment. Front Immunol 11:584903. https:// doi.org/10.3389/fimmu.2020.584903

112. Rabinovich GA, Gabrilovich D, Sotomayor EM (2007) Immunosuppressive strategies that are mediated by tumor cells. Annu Rev Immunol 25:267-296. https://doi.org/10.1146/annurev.immunol.25.022106. 141609

113. Raper J, Murphy L, Richardson R, Romm Z, Kovacs-Balint Z, Payne C, Galvan A (2019) Chemogenetic inhibition of the amygdala modulates. Emot Behav Express Infant Rhesus Monkeys 6:10. https://doi.org/10. 1523/ENEURO.0360-19.2019

114. Razavi R, Chan Y, Afifiyan FN, Liu XJ, Wan X, Yantha J, Tsui H, Tang L, Tsa S, Santamaria P et al (2006) TRPV1 + sensory neurons control beta cell stress and islet inflammation in autoimmune diabetes. Cell 127:11231135. https://doi.org/10.1016/j.cell.2006.10.038

115. Renz BW, Takahashi R, Tanaka T, Macchini M, Hayakawa Y, Dantes Z, Maurer HC, Chen X, Jiang Z, Westphalen CB et al (2018) beta2 adrenergic-neurotrophin feedforward loop promotes pancreatic cancer. Cancer Cell 33(75-90):e77. https://doi.org/10.1016/j.ccell.2017.11.007

116. Renz BW, Tanaka T, Sunagawa M, Takahashi R, Jiang Z, Macchini M, Dantes Z, Valenti G, White RA, Middelhoff MA et al (2018) Cholinergic signaling via muscarinic receptors directly and indirectly suppresses pancreatic tumorigenesis and cancer stemness. Cancer Discov 8:1458-1473. https://doi.org/10.1158/2159-8290.CD-18-0046

117. Rochlitzer S, Veres TZ, Kuhne K, Prenzler F, Pilzner C, Knothe S, Winkler C, Lauenstein HD, Willart M, Hammad H et al (2011) The neuropeptide calcitonin gene-related peptide affects allergic airway inflammation by modulating dendritic cell function. Clin Exp Allergy 41:1609-1621. https://doi.org/10.1111/j.1365-2222.2011.03822.x

118. Rosenberg SA, Packard BS, Aebersold PM, Solomon D, Topalian SL, Toy ST, Simon P, Lotze MT, Yang JC, Seipp CA et al (1988) Use of tumorinfiltrating lymphocytes and interleukin-2 in the immunotherapy of patients with metastatic melanoma. A preliminary report. N Engl J Med 319:1676-1680. https://doi.org/10.1056/NEJM198812223192527

119. Roth BL (2016) DREADDs for neuroscientists. Neuron 89:683-694. https://doi.org/10.1016/ineuron.2016.01.040

120. Ruff MR, Wahl SM, Pert CB (1985) Substance P receptor-mediated chemotaxis of human monocytes. Peptides 6(Suppl 2):107-111. https:// doi.org/10.1016/0196-9781(85)90142-1

121. Saloman JL, Albers KM, Li D, Hartman DJ, Crawford HC, Muha EA, Rhim AD, Davis BM (2016) Ablation of sensory neurons in a genetic model of pancreatic ductal adenocarcinoma slows initiation and progression of cancer. Proc Natl Acad Sci U S A 113:3078-3083. https://doi.org/10. 1073/pnas.1512603113

122. Sanmamed $M F$, Chen $L$ (2018) A paradigm shift in cancer immunotherapy: from enhancement to normalization. Cell 175:313-326. https://doi. org/10.1016/j.cell.2018.09.035

123. Santos GSP, Costa AC, Picoli CC, Rocha BGS, Sulaiman SO, Radicchi DC et al (2021) Sympathetic nerve-adipocyte interactions in response to acute stress. J Mol Med. In press. https://doi.org/10.1007/ s00109-021-02157-0

124. Schadendorf D, Hauschild A (2014) Melanoma in 2013: melanoma-the run of success continues. Nat Rev Clin Oncol 11:75-76. https://doi.org/ 10.1038/nrclinonc.2013.246

125. Schmalhofer WA, Calhoun J, Burrows R, Bailey T, Kohler MG, Weinglass AB, Kaczorowski GJ, Garcia ML, Koltzenburg M, Priest BT (2008) ProTx-II, a selective inhibitor of NaV1.7 sodium channels, blocks action potential propagation in nociceptors. Mol Pharmacol 74:1476-1484. https://doi. org/10.1124/mol.108.047670

126. Schmidt H, Bastholt L, Geertsen P, Christensen IJ, Larsen S, Gehl J, von der Maase H (2005) Elevated neutrophil and monocyte counts in peripheral blood are associated with poor survival in patients with metastatic melanoma: a prognostic model. Br J Cancer 93:273-278. https://doi.org/10.1038/sj.bjc.6602702
127. Sciolino NR, Plummer NW, Chen YW, Alexander GM, Robertson SD, Dudek SM, McElligott ZA, Jensen P (2016) Recombinase-dependent mouse lines for chemogenetic activation of genetically defined cell types. Cell Rep 15:2563-2573. https://doi.org/10.1016/j.celrep.2016.05. 034

128. Sena IFG, Rocha BGS, Picoli CC, Santos GSP, Costa AC, Goncalves BOP, Garcia APV, Soltani-Asl M, Coimbra-Campos LMC, Silva WN et al (2021) C(3)1-TAg in C57BL/6 J background as a model to study mammary tumor development. Histochem Cell Biol. https://doi.org/10.1007/ s00418-021-01995-W

129. Shen M, Hu P, Donskov F, Wang G, Liu Q, Du J (2014) Tumor-associated neutrophils as a new prognostic factor in cancer: a systematic review and meta-analysis. PLoS ONE 9:e98259. https://doi.org/10.1371/journal. pone.0098259

130. Smyth MJ, Teng MW, Swann J, Kyparissoudis K, Godfrey DI, Hayakawa Y (2006) CD4+CD25+ T regulatory cells suppress NK cell-mediated immunotherapy of cancer. J Immunol 176:1582-1587. https://doi.org/ 10.4049/jimmunol.176.3.1582

131. Sternson SM, Roth BL (2014) Chemogenetic tools to interrogate brain functions. Annu Rev Neurosci 37:387-407. https://doi.org/10.1146/ annurev-neuro-071013-014048

132. Szklarczyk D, Gable AL, Lyon D, Junge A, Wyder S, Huerta-Cepas J, Simonovic M, Doncheva NT, Morris JH, Bork P et al (2019) STRING v11: protein-protein association networks with increased coverage, supporting functional discovery in genome-wide experimental datasets. Nucleic Acids Res 47:D607-D613. https://doi.org/10.1093/nar/gky1131

133. Teng MW, Ngiow SF, von Scheidt B, McLaughlin N, Sparwasser T, Smyth MJ (2010) Conditional regulatory T-cell depletion releases adaptive immunity preventing carcinogenesis and suppressing established tumor growth. Cancer Res 70:7800-7809. https://doi.org/10.1158/00085472.CAN-10-1681

134. Teng MW, Swann JB, von Scheidt B, Sharkey J, Zerafa N, McLaughlin N, Yamaguchi T, Sakaguchi S, Darcy PK, Smyth MJ (2010) Multiple antitumor mechanisms downstream of prophylactic regulatory T-cell depletion. Cancer Res 70:2665-2674. https://doi.org/10.1158/00085472. CAN-09-1574

135. Torcellan T, Hampton HR, Bailey J, Tomura M, Brink R, Chtanova T (2017) In vivo photolabeling of tumor-infiltrating cells reveals highly regulated egress of T-cell subsets from tumors. Proc Natl Acad Sci U S A 114:5677-5682. https://doi.org/10.1073/pnas.1618446114

136. Treffers LW, Hiemstra IH, Kuijpers TW, van den Berg TK, Matlung HL (2016) Neutrophils in cancer. Immunol Rev 273:312-328. https://doi. org/10.1111/imr.12444

137. Tucci M, Passarelli A, Mannavola F, Felici C, Stucci LS, Cives M, Silvestris F (2019) Immune system evasion as hallmark of melanoma progression: the role of dendritic cells. Front Oncol 9:1148. https://doi.org/10.3389/ fonc.2019.01148

138. Tuting T, de Visser KE (2016) CANCER. How neutrophils promote metastasis. Science 352:145-146. https://doi.org/10.1126/science.aaf7300

139. Uguen A, Talagas M, Costa S, Duigou S, Bouvier S, De Braekeleer M, Marcorelles P (2015) A p16-Ki-67-HMB45 immunohistochemistry scoring system as an ancillary diagnostic tool in the diagnosis of melanoma. Diagn Pathol 10:195. https://doi.org/10.1186/s13000-015-0431-9

140. Umansky V, Sevko A, Gebhardt C, Utikal J (2014) Myeloid-derived suppressor cells in malignant melanoma. J Dtsch Dermatol Ges 12:10211027. https://doi.org/10.1111/ddg.12411

141. Usoskin D, Furlan A, Islam S, Abdo H, Lonnerberg P, Lou D, HjerlingLeffler J, Haeggstrom J, Kharchenko O, Kharchenko PV et al (2015) Unbiased classification of sensory neuron types by large-scale single-cell RNA sequencing. Nat Neurosci 18:145-153. https://doi.org/10.1038/nn. 3881

142. Vacas E, Arenas MI, Munoz-Moreno L, Bajo AM, Sanchez-Chapado M, Prieto JC, Carmena MJ (2013) Antitumoral effects of vasoactive intestinal peptide in human renal cell carcinoma xenografts in athymic nude mice. Cancer Lett 336:196-203. https://doi.org/10.1016/j.canlet.2013.04 033

143. Vacas E, Fernandez-Martinez AB, Bajo AM, Sanchez-Chapado M, Schally AV, Prieto JC, Carmena MJ (2012) Vasoactive intestinal peptide (VIP) inhibits human renal cell carcinoma proliferation. Biochim Biophys Acta 1823:1676-1685. https://doi.org/10.1016/j.bbamcr.2012.06.018 
144. Vriens J, Owsianik G, Hofmann T, Philipp SE, Stab J, Chen X, Benoit M, Xue F, Janssens A, Kerselaers S et al (2011) TRPM3 is a nociceptor channel involved in the detection of noxious heat. Neuron 70:482-494. https://doi.org/10.1016/j.neuron.2011.02.051

145. Weber R, Fleming V, Hu X, Nagibin V, Groth C, Altevogt P, Utikal J, Umansky $V$ (2018) Myeloid-derived suppressor cells hinder the anticancer activity of immune checkpoint inhibitors. Front Immunol 9:1310. https://doi.org/10.3389/fimmu.2018.01310

146. Wherry EJ, Kurachi M (2015) Molecular and cellular insights into T cell exhaustion. Nat Rev Immunol 15:486-499. https://doi.org/10.1038/ nri3862

147. Whissell PD, Tohyama S, Martin LJ (2016) The use of DREADDs to deconstruct behavior. Front Genet 7:70. https://doi.org/10.3389/fgene.2016. 00070

148. Wojcieszak J, Zawilska JB (2014) PACAP38 and PACAP6-38 exert cytotoxic activity against human retinoblastoma Y79 cells. J Mol Neurosci 54:463-468. https://doi.org/10.1007/s12031-014-0248-0

149. Wolchok JD, Saenger $Y$ (2008) The mechanism of anti-CTLA-4 activity and the negative regulation of T-cell activation. Oncologist 13(Suppl 4):2-9. https://doi.org/10.1634/theoncologist.13-54-2

150. Yang L, DeBusk LM, Fukuda K, Fingleton B, Green-Jarvis B, Shyr Y, Matrisian LM, Carbone DP, Lin PC (2004) Expansion of myeloid immune suppressor $\mathrm{Gr}+\mathrm{CD} 11 \mathrm{~b}+$ cells in tumor-bearing host directly promotes tumor angiogenesis. Cancer Cell 6:409-421. https://doi.org/10.1016/j. ccr.2004.08.031

151. Yin W, Song Y, Liu Q, Wu Y, He R (2017) Topical treatment of all-trans retinoic acid inhibits murine melanoma partly by promoting CD8(+) T-cell immunity. Immunology 152:287-297. https://doi.org/10.1111/ imm.12768

152. Yuan J, Adamow M, Ginsberg BA, Rasalan TS, Ritter E, Gallardo HF, Xu Y, Pogoriler E, Terzulli SL, Kuk D et al (2011) Integrated NY-ESO-1 antibody and CD8 + T-cell responses correlate with clinical benefit in advanced melanoma patients treated with ipilimumab. Proc Natl Acad Sci U S A 108:16723-16728. https://doi.org/10.1073/pnas.1110814108
153. Yuan J, Ginsberg B, Page D, Li Y, Rasalan T, Gallardo HF, Xu Y, Adams S, Bhardwaj N, Busam K et al (2011) CTLA-4 blockade increases antigenspecific CD8(+) T cells in prevaccinated patients with melanoma: three cases. Cancer Immunol Immunother 60:1137-1146. https://doi.org/10. 1007/s00262-011-1011-9

154. Zahalka AH, Arnal-Estape A, Maryanovich M, Nakahara F, Cruz CD, Finley LWS, Frenette PS (2017) Adrenergic nerves activate an angio-metabolic switch in prostate cancer. Science 358:321-326. https://doi.org/10. 1126/science.aah5072

155. Zea AH, Rodriguez PC, Atkins MB, Hernandez C, Signoretti S, Zabaleta J, McDermott D, Quiceno D, Youmans A, O’Neill A et al (2005) Arginaseproducing myeloid suppressor cells in renal cell carcinoma patients: a mechanism of tumor evasion. Cancer Res 65:3044-3048. https://doi. org/10.1158/0008-5472.CAN-04-4505

156. Zenkova D, Kamenev V, Sablina R, Artyomov MAS (2020) Phantasus: visual and interactive gene expression analysis. Science. https://doi.org/ 10.18129/B9.bioc.phantasus

157. Zhang B, Ma S, Rachmin I, He M, Baral P, Choi S, Goncalves WA, Shwartz Y, Fast EM, Su Y et al (2020) Hyperactivation of sympathetic nerves drives depletion of melanocyte stem cells. Nature 577:676-681. https:// doi.org/10.1038/s41586-020-1935-3

158. Zhao CM, Hayakawa Y, Kodama Y, Muthupalani S, Westphalen CB, Andersen GT, Flatberg A, Johannessen H, Friedman RA, Renz BW et al (2014) Denervation suppresses gastric tumorigenesis. Sci Transl Med. https://doi.org/10.1126/scitranslmed.3009569

159. Zhu H, Aryal DK, Olsen RH, Urban DJ, Swearingen A, Forbes S, Roth BL, Hochgeschwender U (2016) Cre-dependent DREADD (Designer Receptors Exclusively Activated by Designer Drugs) mice. Genesis 54:439-446. https://doi.org/10.1002/dvg.22949

\section{Publisher's Note}

Springer Nature remains neutral with regard to jurisdictional claims in published maps and institutional affiliations.
Ready to submit your research? Choose BMC and benefit from:

- fast, convenient online submission

- thorough peer review by experienced researchers in your field

- rapid publication on acceptance

- support for research data, including large and complex data types

- gold Open Access which fosters wider collaboration and increased citations

- maximum visibility for your research: over $100 \mathrm{M}$ website views per year

At $\mathrm{BMC}$, research is always in progress.

Learn more biomedcentral.com/submissions 